\title{
HOPF-ALGEBRAIC TECHNIQUES APPLIED TO SUPER LIE GROUPS OVER A COMPLETE FIELD
}

\author{
MITSUKAZU HOSHI, AKIRA MASUOKA, AND YUTA TAKAHASHI
}

\begin{abstract}
We show basic results on super-manifolds and super Lie groups over a complete field of characteristic $\neq 2$, extensively using Hopf-algebraic techniques. The main results are two theorems. The first main theorem shows a category equivalence between super Lie groups and Harish-Chandra pairs, which is applied especially to construct the Hopf super-algebra of all analytic representative functions on a super Lie group. The second constructs homogeneous super-manifolds by a new Hopf-algebraic method, showing their remarkable property.
\end{abstract}

KEY WoRDS: super Lie group, Harish-Chandra pair, super-manifold, Hopf super-algebra, hyper-super-algebra, affine super-group scheme, principal super bundle

Mathematics Subject Classification (2000): 58A50, 16T05, 14M30, $14 \mathrm{~L} 15$.

\section{INTRODUCTION}

1.1. Our aim and achievements. Varadarajan [24, Section 11] raised four problems for future research on super-symmetry, one of which is "to extend super-symmetry to $p$-adic Lie groups". This paper aims to answer the problem. Indeed, our answer is not total, but we work in the more general situation that the base field is a complete field of characteristic $\neq 2$. A complete field [18, Part II, Chaper I] is a (necessarily infinite) field which is complete with respect to a non-trivial absolute value. The examples include the real- and the complex number fields $\mathbb{R}$ and $\mathbb{C}$, the $p$-adic fields $\mathbb{Q}_{p}$ and their finite extension fields and the formal power series field $F((T))$ in one variable over an arbitrary field $F$.

Choose such a field $\mathbb{k}$ of characteristic $\neq 2$, as our base field. We will prove basic results on super-manifolds and super Lie groups over $\mathbb{k}$. Our main results are Theorem 6.3 and Theorem 7.3 . The former proves a category equivalence between super Lie groups and Harish-Chandra pairs, and produces some consequences including Corollaries 6.13 and 6.15, and Theorem 6.18. The latter Theorem 7.3 constructs homogeneous super-manifolds, showing their remarkable property which is likely new even when $\mathbb{k}=\mathbb{R}$ or $\mathbb{C}$. Preliminaries include discussion of super-manifolds from the scratch since even such basics on those over a complete field cannot be found in the literature. Our method is Hopf-algebraic, featured by (1) frequent use of discrete and topological Hopf algebras together with duality between them, and (2) applications of well-known results on (discrete) Hopf algebras, such as those on Hopf modules; the latter applications in such analytic situation 
of ours might have been unexpected so far. Apart from Hopf algebra another feature is (3) careful treatment of sheaves with close investigation of stalks or their completions. Especially on Hopf (super-)algebras we will give enough details, since they are highly expected to play an important role in further investigations of super Lie groups, as well.

1.2. Basic results on super-manifolds. These will be presented in Section 4. A super-manifold (see Definition 4.1) is a kind of a super-ringed space $X=\left(|X|, \mathcal{O}_{X}\right)$, that is, a topological space $|X|$ equipped with a structure sheaf $\mathcal{O}=\mathcal{O}_{X}$ of super-algebras; this $|X|$, equipped with the associated sheaf $\mathcal{O}_{\text {red }}$ of reduced algebras, turns into an analytic manifold, $X_{\text {red }}=\left(|X|, \mathcal{O}_{\text {red }}\right)$. (Super-algebras are supposed to be super-commutative unless otherwise stated.) Theorem 4.5 is the Inverse Function Theorem proved in our generalized situation; an idea of the proof is to focus on the stalks $\mathcal{O}_{x}, x \in X$, or rather on the associated graded super-algebras $\operatorname{gr} \mathcal{O}_{x}$, and it is used in some other places as well. Proposition 4.6 shows that the category SMFD of super-manifolds has finite products, whence group objects in SMFD are defined. Such a group object is called a super Lie group; see Definition [5.1]

1.3. Super Lie groups and Harish-Chandra pairs. These will be discussed in Sections 56 6. The notion of Harish-Chandra pairs and their category equivalence with super Lie groups in the $C^{\infty}$-situation are attributed to Kostant [7] and Koszul [8; see also [1, Section 7.4]. The analogous category equivalences are proved by Vishnyakova [25], and Carmeli and Fioresi [2] in the complex analytic situation, and by the second-named author [11] (see also [12, 5, 13, 14]) in the algebraic situation; the group objects in the last algebraic situation are affine algebraic super-group schemes over an arbitrary field of characteristic $\neq 2$; see Section 2.2.

Given a super Lie group $G$, the associated analytic manifold $G_{\text {red }}$ naturally turns into a Lie group, which acts on the Lie super-algebra $\operatorname{Lie}(G)$ of $G$ by the right adjoint action. Regard the odd part $\operatorname{Lie}(G)_{1}$ of $\operatorname{Lie}(G)$ as

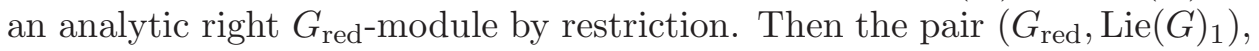
equipped with the bracket [ , ] : $\operatorname{Lie}(G)_{1} \otimes \operatorname{Lie}(G)_{1} \rightarrow \operatorname{Lie}\left(G_{\text {red }}\right)\left(=\operatorname{Lie}(G)_{0}\right)$ restricted from that of $\operatorname{Lie}(G)$, is a Harish-Chandra pair as we define; in fact, our definition of the notion (see Definition 6.1) is different from (though equivalent to) the standard one. The first main theorem of ours, Theorem 6.3, states that $\Phi: G \mapsto\left(G_{\text {red }}, \operatorname{Lie}(G)_{1}\right)$ gives an equivalence from the category SLG of super Lie groups to the category HCP of Harish-Chandra pairs. To prove this, essential is to construct a super Lie group from a given Harish-Chandra pair, which shall lead to a quasi-inverse of the functor $\Phi$. Although the super Lie group we construct turns out to be essentially the same as the ones constructed by Carmeli et al. [1, 2] in the $C^{\infty}$ - and the complex analytic situations, we choose the (hopefully, rigorous) manner of constructing it from a certain Hopf super-algebra $\mathcal{B}$ associated with the given Harish-Chandra pair; see Section 6.2. The Hopf super-algebra $\mathcal{B}$ was already constructed in [13, 11]. But we will re-produce the construction because one could then realize the construction (of $\mathcal{B}$, and hence of the super Lie group as well) to be quite natural, and because it is used to prove the subsequent 
Theorem 6.18, this theorem shows that given a super Lie group $G$, the associated $\mathcal{B}$ is the Hopf super-algebra of analytic representative functions on $G$ in the sense that the category $G$-SMod of analytic $G$-super-modules is identified with the category $\mathrm{SComod}-\mathcal{B}$ of $\mathcal{B}$-super-comodules. We would emphasize: once one defines the notion of Harish-Chandra pairs as we do, it is quite natural to formulate and to prove the theorem.

Corollary 6.15, a corollary to Theorem 6.3, constructs a natural functor from the category of affine algebraic super-group schemes satisfying some additional condition to the category of super Lie groups. One sometimes encounters such presentations of real or complex super Lie groups that look presenting, by use of matrices, affine algebraic super-group schemes. The last mentioned functor justifies the presentations (under some mild restriction in the real case), since they can be understood to indicate the super Lie groups which arise through the functor from the actually presented supergroup schemes.

1.4. Quotients $G / H$. Their construction will be done in the final Section 7

The second main theorem, Theorem [7.3, constructs the quotient supermanifold $G / H$, where $G$ is a super Lie group and $H$ is a super Lie subgroup of $G$. The construction of ours is rather Hopf-algebraic, and should be new. Moreover, we will show a remarkable property of $G / H$, which is likely new even when $\mathbb{k}=\mathbb{R}$ or $\mathbb{C}$. To describe the property we remark that the Lie group $H_{\text {red }}$ associated with $H$ is a Lie subgroup of the Lie group $G_{\text {red }}$ associated with $G$, so that the quotient manifold $G_{\text {red }} / H_{\text {red }}$ is constructed. In addition, the underlying topological space $|G / H|$ of $G / H$ coincides with that space $\left|G_{\text {red }} / H_{\text {red }}\right|$ of $G_{\text {red }} / H_{\text {red }}$. It is known that the classical quotient $\pi: G_{\text {red }} \rightarrow G_{\text {red }} / H_{\text {red }}$ is a principal $H_{\text {red-bundle. This means every point }}$ of $\left|G_{\text {red }} / H_{\text {red }}\right|$ is contained in an open set $U$ such that there exists a right

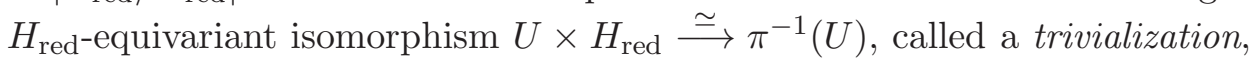
which is compatible with the natural epimorphisms onto $U$. The property we will show is that every trivialization such as above can lift to one in the super context. Or more precisely, there exists a right $H$-equivariant isomorphism $\left(U,\left.\mathcal{O}_{G / H}\right|_{U}\right) \times H \stackrel{\simeq}{\longrightarrow}\left(\pi^{-1}(U),\left.\mathcal{O}_{G}\right|_{\pi^{-1}(U)}\right)$ of super-manifolds which is compatible with the natural epimorphisms onto $\left(U,\left.\mathcal{O}_{G / H}\right|_{U}\right)$ and which is reduced to the original trivialization; note that $U$ is here regarded an open set of $|G / H|$. To prove this, Hopf modules as well as the construction of the quasi-inverse of the functor $\Phi$ above play a role. The proof is given by analogous argument of proving [15, Theorem 4.12], a very recent result by the second- and the third-named authors; it turns to have proved an analogous result to our Theorem 7.3 in the algebraic situation mentioned above at the first paragraph of Section 1.2. See Remark 7.4,

1.5. Key duality. The next Section 2 is devoted to preliminaries, which include introducing topological super-(co)modules; see Section 2.4. The following Section 3 discusses duality between complete local super-algebras and connected super-coalgebras. Theorem 3.15 proves an anti-equivalence between the category of Noetherian complete (topological) Hopf super-algebras and the category of connected Hopf super-algebras of finite type. (The latter connected Hopf super-algebras may not be super-commutative, and are 
called hyper-super-algebras; see Definition 3.7.) An important consequence of this and Proposition 2.3 is that a topological super-comodule over a Noetherian complete Hopf super-algebra is identified with a topological supermodule over the corresponding connected Hopf super-algebra of finite type. Let $G$ be a super Lie group with structure sheaf $\mathcal{O}$. Then the completion $\hat{\mathcal{O}}_{e}$ of the stalk $\mathcal{O}_{e}$ at the identity element $e$ is a Noetherian complete Hopf super-algebra. The corresponding finite-type connected Hopf super-algebra is the hyper-super-algebra hy $(G)$ of $G$ (see Definition [5.6); it coincides with the universal envelope of $\operatorname{Lie}(G)$ in characteristic zero. The identification mentioned above is used effectively, for example, when we prove in Corollary 5.15 that $\mathcal{O}$ is a sheaf of hy $(G)$-super-module super-algebras.

\section{Preliminaries}

Suppose that $\mathbb{k}$ is a field of characteristic $\neq 2$. Throughout we work over it. Vector spaces and linear maps are thus those over $\mathbb{k} ; \otimes$ denotes the tensor product over $\mathbb{k}$; Hom indicates the vector space of all $\mathbb{k}$-linear maps.

2.1. Super-vector spaces. A super-vector space is a vector space $V=$ $\mathrm{V}_{0} \oplus \mathrm{V}_{1}$ graded by the order 2 group $\mathbb{Z}_{2}=\{0,1\}$ which consists of the even 0 and the odd 1. It is said to be purely even (resp., purely odd) if $\mathrm{V}=\mathrm{V}_{0}$ (resp., if $\mathrm{V}=\mathrm{V}_{1}$ ). The super-vector spaces and the super- (or $\mathbb{Z}_{2}$-graded) linear maps form a symmetric tensor category, SMod $d_{\mathbb{k}}$, with respect to the obvious tensor product $\mathrm{V} \otimes \mathrm{W}$ and the so-called super-symmetry

$$
\tau=\tau_{\mathrm{V}, \mathrm{W}}: \mathrm{V} \otimes \mathrm{W} \rightarrow \mathrm{W} \otimes \mathrm{V}, \tau(v \otimes w)=(-1)^{|v||w|} w \otimes v,
$$

where $v \in \mathrm{V}$ and $w \in \mathrm{W}$ are homogeneous elements of degree $|v|$ and $|w|$, respectively. The unit object is the purely even $\mathbb{k}$. The ordinary objects, such as Lie algebra or Hopf algebra, defined in the tensor category of vector spaces based on its trivial symmetry are generalized by the "super" objects defined in SMod ${ }_{\mathbb{k}}$, which are called with the adjective "super" attached, so as Lie super-algebra or Hopf super-algebra. Ordinary objects are precisely purely even super-objects.

Let $\mathfrak{g}=\mathfrak{g}_{0} \oplus \mathfrak{g}_{1}$ be a Lie super-algebra, to give a remark on its definition. If char $\mathbb{k}=3$, we require the associated bracket [, ]: $\mathfrak{g} \otimes \mathfrak{g} \rightarrow \mathfrak{g}$ to satisfy (B3) in addition to (B1) and (B2), below.

(B1) $[,] \circ\left(\mathrm{id}_{\mathfrak{g} \otimes \mathfrak{g}}+\tau_{\mathfrak{g}, \mathfrak{g}}\right)=0$,

(B2) $[[],] \circ\left(\operatorname{id}_{\mathfrak{g} \otimes \mathfrak{g} \otimes \mathfrak{g}}+\tau_{\mathfrak{g}, \mathfrak{g} \otimes \mathfrak{g}}+\tau_{\mathfrak{g} \otimes \mathfrak{g}, \mathfrak{g}}\right)=0$,

(B3) $[[v, v], v]=0, v \in \mathfrak{g}_{1}$.

See [13, Section 3.1], for example. If char $\mathbb{k} \neq 3$, then (B2) implies (B3).

2.2. Hopf super-algebras and affine super-group schemes. A super(co) algebra is precisely a $\mathbb{Z}_{2}$-graded (co)algebra.

A super-algebra $A=A_{0} \oplus A_{1}$ is said to be super-commutative if the product $A \otimes A \rightarrow A, a \otimes b \mapsto a b$ is unchanged, composed with $\tau_{A, A}$, or more explicitly, if $A_{0}$ is central in $A$ and if arbitrary two elements $a, b \in A_{1}$ are anti-commutative, or $a b=-b a$. We set the following.

Convention 2.1. Super-algebras are supposed to be super-commutative unless otherwise stated. 
Accordingly, algebras are supposed to be commutative.

For a super-coalgebra $C$, the coproduct and the counit are denoted by

$$
\Delta=\Delta_{C}: C \rightarrow C \otimes C, \Delta(c)=c_{(1)} \otimes c_{(2)} \text { and } \epsilon=\epsilon_{C}: C \rightarrow \mathbb{k},
$$

respectively. We use the sigma notation [21, Section 1.2] as above, to present $\Delta(c)$. A super-coalgebra is said to be super-cocommutative if $\Delta=\tau_{C, C} \circ \Delta$.

The Hopf super-algebras we will consider are super-commutative or supercocommutative; we emphasize that they may not be super-commutative. The antipode of a Hopf super-algebra, say $C$, is denoted by $S=S_{C}: C \rightarrow C$. We let $\mathbb{N}=\{0,1,2, \ldots\}$ denote the monoid of non-negative integers. An $\mathbb{N}$ graded Hopf super-algebra is an algebra and coalgebra graded by $\mathbb{N}$ which, regarded by the mod 2 reduction as a super-algebra and super-coalgebra, is a Hopf super-algebra.

An affine super-group scheme is a representable group-valued functor G defined on the category of super-algebras. It is uniquely represented by a super-commutative Hopf super-algebra, which we denote by $O_{\mathrm{G}}$. Thus, G is the functor which associates to each super-algebra $R$, the group $\operatorname{SAlg}\left(O_{\mathrm{G}}, R\right)$ of all super-algebra maps $O_{\mathrm{G}} \rightarrow R$ with respect to the convolution-product [17, Definition 1.4.1]. This $\mathrm{G}$ is called an affine algebraic super-group scheme if $O_{\mathrm{G}}$ is finitely generated.

Suppose that $C$ is a Hopf super-algebra. We let

$$
C \text {-SMod, SMod- } C \text { (resp., } C \text {-SComod, SComod- } C \text { ) }
$$

denote the tensor categories of left $C$-super-modules and of right $C$-supermodules (resp., of left $C$-super-comodules and of right $C$-super-comodules). A (co)algebra object in $C$-SMod is called a left $C$-super-module super-(co)algebra; cf. [17, p.203]. Note that the last definition involves the tensor product of super-(co)algebras, and hence the super-symmetry. The categories above are symmetric with respect to the super-symmetry if $C$ is super-cocommutative (resp., super-commutative); in fact, the symbols will be used only when that is the case, and in addition, $C$ is purely even. Note that if $C$ is purely even, then a $C$-super-(co)module is precisely a super-vector space whose homogeneous components are both $C$-(co)modules.

Given a super-vector space $\mathrm{V}=\mathrm{V}_{0} \oplus \mathrm{V}_{1}$, we let $\mathrm{V}^{*}=\operatorname{Hom}(\mathrm{V}, \mathbb{k})$ denote the dual vector space; it is a super-vector space with respect to the parity $\left(\mathrm{V}^{*}\right)_{i}=$ $\left(\mathrm{V}_{i}\right)^{*}, i=0,1$. When we discuss duality concerning Hopf super-algebras there are two choices, which are, however, equivalent (more precisely, the resulting difference is made up by category equivalences), as was shown in [14. Let $W$ be another super-vector space, and suppose that you have to define a pairing $\langle\rangle:, \mathrm{V}^{*} \otimes \mathrm{W}^{*} \times \mathrm{V} \otimes \mathrm{W} \rightarrow \mathbb{k}$. The choices depend on whether you define $\left\langle v^{*} \otimes w^{*}, v \otimes w\right\rangle$ to be

$$
v^{*}(v) w^{*}(w) \quad \text { or } \quad(-1)^{|v||w|} v^{*}(v) w^{*}(w),
$$

where $v \in \mathrm{V}, w \in \mathbf{W}, v^{*} \in \mathrm{V}^{*}$ and $w^{*} \in \mathbf{W}^{*}$. We choose the first, simpler one, as the article [11] does with explicit citation. Consequently, for example, the dual super-algebra $C^{*}$ of a super-coalgebra $C$ coincides with the dual algebra [21, Section 1.1] constructed as usually, when one regards $C$ as an ordinary coalgebra. 
2.3. (Graded) algebras associated with a super-algebra. Let $A=$ $A_{0} \oplus A_{1}$ be a super-algebra, which is super-commutative by Convention 2.1 .

Let $I_{A}=\left(A_{1}\right)$ denote the super- (or homogeneous) ideal generated by the odd component $A_{1}$. Define

$$
\bar{A}:=A / I_{A}, \quad \operatorname{gr} A:=\bigoplus_{n \geq 0} I_{A}^{n} / I_{A}^{n+1}\left(=\bar{A} \oplus I_{A} / I_{A}^{2} \oplus \ldots\right) .
$$

Then $\bar{A}$ is the largest purely even quotient super-algebra of $A$, and $\operatorname{gr} A$ is an $\mathbb{N}$-graded algebra, which is super-commutative, regarded as a super-algebra by the mod 2 reduction. Moreover, gr gives rise to a functor from the category of (super-commutative) super-algebras to the category of supercommutative $\mathbb{N}$-graded algebras.

Since every odd element of $A$ is square-zero, it follows that the set $\sqrt{0}$ of all nilpotent elements in $A$ turns into a super-ideal including $I_{A}$. Define

$$
A_{\text {red }}:=A / \sqrt{0} \text {. }
$$

One sees that $A_{\text {red }}$ is the largest reduced quotient algebra of $A$, and that $A \mapsto A_{\text {red }}$ gives rise to a functor. In the (mostly, geometric) circumstances where we will apply the construction, $\bar{A}$ is already reduced, whence $\bar{A}=$ $A_{\text {red. }}$. But we then prefer to use the notation (2.3), following custom.

Remark 2.2. In fact, the construction of gr will be used in the special situation that $A=\operatorname{gr} A$, and $I_{A}\left(=\bigoplus_{n>0} A(n)\right)$ is nilpotent, whence $A(n)=$ 0 for $n \gg 0$. For another such super-algebra $B$, we will consider a superalgebra map $f: A \rightarrow B$. Note that gr $f: A \rightarrow B$ may be different from $f$, but it is an isomorphism if and only if $f$ is.

2.4. Topological super-vector spaces. An important role will be played by an obvious super-analogue of the concept of topological vector spaces [23, p.507], defined as follows. A topological super-vector space is a super-vector space $\mathrm{V}$ equipped with a topology such that

(i) for every $v \in \mathrm{V}$, the translation $w \mapsto v+w$ is continuous, and

(ii) there exists a base $\left(V_{\alpha}\right)_{\alpha}$ for neighborhoods of 0 which consists of super-vector subspaces $\mathrm{V}_{\alpha}$ of $\mathrm{V}$; such a base will be called a topological base.

Let $\mathrm{V}$ and $\left(\mathrm{V}_{\alpha}\right)_{\alpha}$ be as above. The completion of $\mathrm{V}$ is defined by

$$
\hat{\mathrm{V}}=\lim _{\longleftarrow \alpha} \mathrm{V} / \mathrm{V}_{\alpha} \text {. }
$$

The definition does not depend on choice of topological bases. This $\hat{V}$ is a topological super-vector space with the topological base consisting of all

$$
\hat{\mathrm{V}}_{\alpha}=\lim _{\longleftarrow}\left(\mathrm{V}_{\alpha}+\mathrm{V}_{\beta}\right) / \mathrm{V}_{\beta} \text {. }
$$

Alternatively, $\hat{V}$ is the projective limit of the discrete super-vector spaces $\mathrm{V} / \mathrm{V}_{\alpha}$. We have a canonical, continuous super-linear map $\mathrm{V} \rightarrow \hat{\mathrm{V}}$. If this is an isomorphism, then $\mathrm{V}$ is said to be complete. For example, every discrete topological super-vector space, that has the single $\{0\}$ as its topological base, is complete.

Given another topological super-vector space $W$ with a topological base $\left(\mathrm{W}_{\beta}\right)_{\beta}$, the tensor product $\mathrm{V} \otimes \mathrm{W}$ is a topological super-vector space with 
the topological base $\left(\mathrm{V}_{\alpha} \otimes \mathrm{W}+\mathrm{V} \otimes \mathrm{W}_{\beta}\right)_{\alpha, \beta}$; see [23, p.509]. The topology does not depend of choice of topological bases. The completion of $\mathrm{V} \otimes \mathrm{W}$ is denoted by $\mathrm{V} \hat{\otimes} \mathrm{W}$, and is called the complete tensor product of $\mathrm{V}$ and $\mathrm{W}$. We thus have

$$
\mathrm{V} \hat{\otimes} \mathrm{W}=\lim _{\alpha, \beta} \mathrm{V} / \mathrm{V}_{\alpha} \otimes \mathrm{W} / \mathrm{W}_{\beta} .
$$

Note that $\mathrm{V} / \mathrm{V}_{\alpha}=\hat{\mathrm{V}} / \hat{\mathrm{V}}_{\alpha}$, whence $\mathrm{V} \hat{\otimes} \mathrm{W}=\hat{\mathrm{V}} \hat{\otimes} \hat{\mathrm{W}}$. The complete tensor product $f \hat{\otimes} g$ of continuous super-linear maps $f$ and $g$ is the completion of the continuous super-linear map $f \otimes g$.

If $\mathrm{W}$ is discrete and finite-dimensional, then we have

$$
\mathrm{V} \hat{\otimes} \mathrm{W}=\hat{\mathrm{V}} \otimes \mathrm{W} .
$$

When regarded as a (purely even) super-vector space, $\mathbb{k}$ is always supposed to be discrete. Hence, in particular,

$$
\mathrm{V} \hat{\otimes} \mathbb{k}=\hat{\mathrm{V}}=\mathbb{k} \hat{\otimes} \mathrm{V} \text {. }
$$

See [23, (1.9), p.510].

The notion of complete topological coalgebras defined in [23, p.510] is directly generalized to the super context as follows. A complete topological super-vector space $C$ equipped with continuous super-linear maps $\Delta: C \rightarrow$ $C \hat{\otimes} C, \epsilon: C \rightarrow \mathbb{k}$ is called a complete topological super-coalgebra, if $\Delta$ and $\epsilon$ satisfy the coalgebra axioms which are modified from the ordinary, discrete situation with $\otimes$ replaced by $\hat{\otimes}$. Note that the counit property then makes sense since we have $C \hat{\otimes} \mathbb{k}=C=\mathbb{k} \hat{\otimes} C$ by (2.5).

Let $R$ be a super-algebra which may not be super-commutative, and let $m: R \otimes R \rightarrow R$ and $u: \mathbb{k} \rightarrow R$ denote the product and the unit, respectively. Let $\left(R_{\gamma}\right)_{\gamma}$ denote the system of all finite-dimensional super-subspaces. Then $R^{*}$ is a complete topological super-vector space with the topological base $\left(\left(R / R_{\gamma}\right)^{*}\right)_{\gamma}$, so that we have

$$
R^{*} \hat{\otimes} R^{*}=\lim _{\longleftarrow} R_{\gamma}^{*} \otimes R_{\gamma}^{*}=(R \otimes R)^{*} .
$$

Moreover, $m^{*}: R^{*} \rightarrow(R \otimes R)^{*}=R^{*} \hat{\otimes} R^{*}$ and $u^{*}: R^{*} \rightarrow \mathbb{k}$ are continuous maps, with which $R^{*}$ is a complete topological super-coalgebra; see [23, Example 1.15].

Let $\mathrm{V}$ be a complete topological super-vector space with a topological base $\left(\mathrm{V}_{\alpha}\right)_{\alpha}$. A topological right $R^{*}$-super-comodule structure on $\mathrm{V}$ is a continuous super-linear map $\rho: \mathrm{V} \rightarrow \mathrm{V} \hat{\otimes} R^{*}$ that satisfies the the right-comodule axioms modified as before; see [23, p.512]. Note that

$$
\mathrm{V} \hat{\otimes} R^{*}=\lim _{\leftarrow, \gamma} \operatorname{Hom}\left(R_{\gamma}, \mathrm{V} / \mathrm{V}_{\alpha}\right)=\operatorname{Hom}(R, \mathrm{~V})
$$

Therefore, given a topological right $R^{*}$-super-comodule structure $\rho$ on $\mathrm{V}$ as above, there arises a super-linear map $\rho^{\prime}: R \otimes \mathrm{V} \rightarrow \mathrm{V}$ which is adjoint to the $\rho: \mathrm{V} \rightarrow \operatorname{Hom}(R, \mathrm{~V})$ above.

Proposition 2.3 ([23, Theorem 1.19]). This $\rho^{\prime}$ is a left $R$-super-module structure on $\mathrm{V}$ which is topological in the sense that every element of $R$ acts on $\mathrm{V}$ as a continuous super-linear endomorphism. Moreover, $\rho \mapsto \rho^{\prime}$ gives a bijection from the set of all topological right $R^{*}$-super-comodule structures on $\mathrm{V}$ to the set of all topological left $R$-super-module structures on $\mathrm{V}$. 


\section{Complete Hopf Super-Algebras AND HYPer-Super-Algebras}

We continue to suppose that $\mathbb{k}$ is a field of characteristic $\neq 2$.

3.1. Local super-algebras. Let $A=A_{0} \oplus A_{1}$ be a super-algebra. Recall the following definitions; see [16, Appendix], for example. A is Noetherian, if the super-ideals of $A$ satisfies the $\mathrm{ACC}$, or equivalently, if the algebra $A_{0}$ is Noetherian and the $A_{0}$-algebra $A$ is generated by finitely many odd elements. $A$ is smooth, if given a super-algebra surjection $B \rightarrow C$ with nilpotent kernel, every super-algebra map $A \rightarrow C$ factors through the given surjection. $A$ is local, if it is non-zero and has a unique maximal super-deal; we denote this maximal by $\mathfrak{m}_{A}$. The condition is equivalent to that the algebra $A_{0}$ is local, and as well, to that $\bar{A}$ (see (2.2) ) is local. By convention a local super-algebra $A$ is regarded as a topological super-vector space with the topological base $\left\{\mathfrak{m}_{A}^{n}\right\}_{n \geq 0}$, or namely, with the $\mathfrak{m}_{A}$-adic topology. It is, therefore, complete if and only if the canonical map

$$
A \rightarrow \hat{A}=\lim _{\longleftarrow} A / \mathfrak{m}_{A}^{n}
$$

is isomorphic. In general, this canonical map is injective if $A$ is Noetherian. In addition, $\hat{A}$ is a complete local super-algebra with the maximal superideal

$$
\hat{\mathfrak{m}}_{A}=\lim _{\longleftarrow} \mathfrak{m}_{A} / \mathfrak{m}_{A}^{n} .
$$

By a connected super-algebra we mean a local super-algebra $A$ with the residue field $A / \mathfrak{m}_{A}=\mathbb{k}$.

Remark 3.1. A super-algebra map $f: A \rightarrow B$ between local super-algebras is continuous if and only if $f\left(\mathfrak{m}_{A}\right) \subset \mathfrak{m}_{B}$. This is necessarily the case if $B$ is connected. In fact, the composite $A \rightarrow B / \mathfrak{m}_{B}=\mathbb{k}$ of $f$ with the projection $B \rightarrow B / \mathfrak{m}_{B}$ then must be a surjection with kernel $\mathfrak{m}_{A}$, whence $A$ is connected, too.

Given non-negative integers $m$ and $n$, the formal power series superalgebra

$$
\mathbb{k}\left[\left[\mathrm{T}_{1}, \ldots, \mathrm{T}_{m+n}\right]\right]
$$

in $m$ even and $n$ odd variables is the tensor product

$$
\mathbb{k}\left[\left[\mathrm{T}_{1}, \ldots, \mathrm{T}_{m}\right]\right] \otimes \wedge\left(\mathrm{T}_{m+1}, \ldots, \mathrm{T}_{m+n}\right)
$$

of the formal power series algebra $\mathbb{k}\left[\left[\mathrm{T}_{1}, \ldots, \mathrm{T}_{m}\right]\right]$ in even variables $\mathrm{T}_{1}, \ldots, \mathrm{T}_{m}$ and the exterior algebra $\wedge\left(\mathrm{T}_{m+1}, \ldots, \mathrm{T}_{m+n}\right)$ on the purely odd super-vector space with a basis $\mathrm{T}_{m+1}, \ldots, \mathrm{T}_{m+n}$.

Proposition 3.2. $\mathbb{k}\left[\left[\mathrm{T}_{1}, \ldots, \mathrm{T}_{m+n}\right]\right]$ is a smooth Noetherian complete connected super-algebra. Conversely, a super-algebra A with these properties is isomorphic to such a formal power series super-algebra for some $m, n \geq 0$.

Proof. This follows easily from [19, Proposition on Page 81]; see also [16, Appendix].

Given local super-algebras $A$ and $B$, their complete tensor product is the super-algebra

$$
A \hat{\otimes} B=\lim _{\longleftarrow} A / \mathfrak{m}_{A}^{n} \otimes B / \mathfrak{m}_{B}^{n} .
$$


If $A$ and $B$ are connected, then $A \hat{\otimes} B$ is, too.

Given continuous super-algebra maps $f: A \rightarrow A^{\prime}$ and $g: B \rightarrow B^{\prime}$ between local super-algebras, the compete tensor product $f \hat{\otimes} g: A \hat{\otimes} B \rightarrow A^{\prime} \hat{\otimes} B^{\prime}$ is a continuous super-algebra map.

3.2. Complete Hopf super-algebras. Let $A$ be a complete connected super-algebra, and let $\epsilon: A \rightarrow A / \mathfrak{m}_{A}=\mathbb{k}$ denote the natural projection.

Definition 3.3. Given a (necessarily continuous) super-algebra map $\Delta$ : $A \rightarrow A \hat{\otimes} A$, we say that $A=(A, \Delta, \epsilon)$ is a complete (topological) Hopf super-algebra, if $\Delta$ and $\epsilon$, as coproduct and counit, respectively, satisfy the coalgebra axioms modified as before.

Remark 3.4. (1) A complete Hopf super-algebra is a complete topological super-coalgebra, in particular.

(2) Every complete Hopf super-algebra has a unique super-algebra involution that acts as antipode; see [9, Proposition 3.4]. A map of complete Hopf super-algebras is a super-algebra map compatible with the coproduct, which is necessarily compatible with the counit and the antipode.

(3) For simplicity and most of the needs of the paper, the above definition of complete Hopf super-algebras, assuming that the underlying complete super-algebras are connected, was made more restrictive than would be expected; indeed, it excludes discrete Hopf super-algebras, for example. The expected one would be a complete super-vector space $A$ equipped with continuous super-linear maps

$$
m: A \hat{\otimes} A \rightarrow A, u: \mathbb{k} \rightarrow A, \Delta: A \rightarrow A \hat{\otimes} A, \epsilon: A \rightarrow \mathbb{k}, S: A \rightarrow A
$$

which satisfy the Hopf-algebra axioms. This version of the notion will be exceptionally used only in a few places, referred to as a complete Hopf superalgebra in the wider sense. A Hopf super-algebra map between those objects is a continuous super-algebra map compatible with the coproduct and the counit; it is necessarily compatible with the antipode.

We see easily the following.

Lemma 3.5. Complete connected super-algebras and super-algebra maps form a category, which has finite coproducts given by $\hat{\otimes}$ and the initial object $\mathbb{k}$. Co-group objects in the category are precisely complete Hopf superalgebras.

Remark 3.6. A smooth Noetherian complete Hopf super-algebra $A$ is defined on some formal power series super-algebra $\mathbb{k}\left[\left[\mathrm{T}_{1}, \ldots, \mathrm{T}_{m+n}\right]\right]$ in $m$ even and $n$ odd variables, as in (3.1). Note that $\epsilon\left(\mathbf{T}_{i}\right)=0,1 \leq i \leq m+n$. We write simply as $A=\mathbb{k}[[\mathrm{T}]]$ with $\mathrm{T}=\left(\mathrm{T}_{1}, \ldots, \mathrm{T}_{m+n}\right)$. Then we can write as $A \hat{\otimes} A=\mathbb{k}[[\mathrm{T}, \mathrm{P}]]$, where $\mathrm{T}$ is as above, and $\mathrm{P}=\left(\mathrm{P}_{1}, \ldots, \mathrm{P}_{m+n}\right)$ is another sequence of $m$ even and $n$ odd variables. Defining $\Delta$ on $A$ is the same as giving, as values $\left(\Delta\left(\mathrm{T}_{1}\right), \ldots, \Delta\left(\mathrm{T}_{m+n}\right)\right)$, a sequence

$$
F(\mathrm{~T}, \mathrm{P})=\left(F_{1}(\mathrm{~T}, \mathrm{P}), \ldots, F_{m+n}(\mathrm{~T}, \mathrm{P})\right)
$$

of elements $F_{i}(\mathrm{~T}, \mathrm{P}) \in \mathbb{k}[[\mathrm{T}, \mathrm{P}]]$ which are even for $i \leq m$, and odd for $i>m$, such that

(i) $F(\mathrm{~T}, 0)=\mathrm{T}, F(0, \mathrm{P})=\mathrm{P}$, 
(ii) $F(F(\mathrm{~T}, \mathrm{P}), \mathrm{Q})=F(\mathrm{~T}, F(\mathrm{P}, \mathrm{Q}))$ in $\mathbb{k}[[\mathrm{T}, \mathrm{P}, \mathrm{Q}]]$.

In view of the definition of formal group laws [18, Page 111], a sequence $F(\mathrm{~T})$ as above may be called a formal super-group law.

3.3. Connected super-coalgebras and hyper-super-algebras. Let $C$ be a super-cocommutative super-coalgebra. Regarded as an ordinary coalgebra, it has the coradical filtration [21, p.185]

$$
C_{(0)} \subset C_{(1)} \subset C_{(2)} \subset \ldots
$$

which starts with the coradical $C_{(0)}=\operatorname{Corad} C$ of $C$, and amounts to $\bigcup_{n>0} C_{(n)}=C$. By definition $C_{(0)}$ is the direct sum of all simple subcoalgebras, each of which is the dual $L^{*}$ of some finite field extension $L / \mathbb{k}$; it is, therefore, purely even. For $n>0, C_{(n)}$ is the kernel of the composite

$$
C \stackrel{\Delta_{n}}{\longrightarrow} C^{\otimes(n+1)} \longrightarrow\left(C / C_{(0)}\right)^{\otimes(n+1)}
$$

of the $n$-times iterated coproduct $\Delta_{n}$ with the natural projection; it is a super-subcoalgebra of $C$. Moreover, we have $\Delta\left(C_{(n)}\right) \subset \sum_{i=0}^{n} C_{(i)} \otimes C_{(n-i)}$. Note that the composite above induces injections

$$
C_{(n)} / C_{(n-1)} \hookrightarrow\left(C_{(1)} / C_{(0)}\right)^{\otimes n}, \quad n>0 .
$$

We say that $C$ is smooth, if given an inclusion $D \subset E$ of super-cocommutative super-coalgebras such that $D \supset$ Corad $E$, every super-coalgebra map $D \rightarrow C$ extends to such a map $E \rightarrow C$.

We say that $C$ is connected, if $\operatorname{dim} C_{(0)}=1$. Suppose that this is the case. Then $C_{(0)}$ is uniquely spanned by a group-like; we denote this grouplike by $1_{C}$. Every super-coalgebra map between connected super-coalgebras preserves the group-like. Let

$$
P(C)=\left\{x \in C \mid \Delta(x)=1_{C} \otimes x+x \otimes 1_{C}\right\}
$$

denote the super-vector subspace of $C$ consisting of all primitives. Then we have

$$
C_{(1)}=\mathbb{k} 1_{C} \oplus P(C) .
$$

See [21, Proposition 10.0.1].

A connected super-coalgebra $C$ is said to be of finite type, if $\operatorname{dim} P(C)<$ $\infty$, or equivalently, if $\operatorname{dim} C_{(n)}<\infty$ for all $n>0$; see (3.3).

Definition 3.7. A hyper-super-algebra is a super-bialgebra which is connected as a super-coalgebra. It may not be super-commutative as a superalgebra.

Remark 3.8 (added in revision). Historically, hyper-algebras, which were eariler called hyper-Lie-algebras, were first intensively studied by M. Takeuchi 22 as objects which play a generalized role of Lie algebras in arbitrary characteristic. The super analogue defined as above has been already studied by several authors (see [11, for example), but in most cases unlike in the present paper, the super objects associated with super Lie (or algebraic) groups (see Definition 5.6 below) are only studied under the alternative name (Hopf) super-algebras of distributions supported at the identity; see [1], for example. 
Making a connected super-coalgebra $C$ into a hyper-super-algebra is the same as giving a super-coalgebra map $C \otimes C \rightarrow C$ which together with the group-like $1_{C}$ make $C$ into an algebra as its product and identity element, respectively.

Every hyper-super-algebra has an antipode, and is, therefore, a Hopf super-algebra. We now see from [21, Corollary 11.0.6] the following.

Lemma 3.9. The connected super-coalgebras and the super-coalgebra maps form a category, which has finite products given by $\otimes$ and the terminal object $\mathbb{k}$. Group objects in the category are precisely hyper-super-algebras.

Let $C$ be a hyper-super-algebra. Set $\mathfrak{g}=P(C)$. Then this super-vector subspace of $C$ is closed under the super-commutator

$$
[x, y]=x y-(-1)^{|x||y|} y x,
$$

with which $\mathfrak{g}$ forms a Lie super-algebra. The universal envelope $\mathfrak{U}(\mathfrak{g})$ of $\mathfrak{g}$ is a hyper-super-algebra in which every element of $\mathfrak{g}$ is primitive. The embedding $\mathfrak{g} \rightarrow C$ uniquely extends to a map

$$
\mathfrak{U}(\mathfrak{g}) \rightarrow C
$$

of hyper-super-algebras, which is an isomorphism if char $\mathbb{k}=0$; this last fact will be re-stated in Proposition 3.13 .

Example 3.10. The exterior algebra $\wedge(\mathrm{V})$ on a vector space $\mathrm{V}$ is a supercommutative hyper-super-algebra in which every element of $V$ is an odd primitive; it is in fact an $\mathbb{N}$-graded Hopf super-algebra with respect to the natural grading. If $\operatorname{dim} \mathrm{V}<\infty$, then the natural parings $\langle\rangle:, \wedge^{n}\left(\mathrm{~V}^{*}\right) \times$ $\wedge^{n}(\mathrm{~V}) \rightarrow \mathbb{k}, n \geq 0$, given by $\langle 1,1\rangle=1$ and

$$
\left\langle v_{1}^{*} \wedge \cdots \wedge v_{n}^{*}, v_{1} \wedge \cdots \wedge v_{n}\right\rangle=\operatorname{det}\left(v_{i}^{*}\left(v_{j}\right)\right),
$$

where $n>0$ and $v_{i}^{*} \in \mathrm{V}^{*}, v_{i} \in \mathrm{V}, 1 \leq i \leq n$, induce an isomorphism

$$
\wedge\left(\mathrm{V}^{*}\right) \simeq \wedge(\mathrm{V})^{*}
$$

of $\mathbb{N}$-graded Hopf super-algebras, through which we will identify the two objects; see [11, Eq.(5), Remark 1], for example.

3.4. Smoothness criteria. Let $U=U_{0} \oplus U_{1}$ be a super-vector space. A connected super-coalgebra on $\mathrm{U}$ is a pair $(C, \pi)$ of a connected supercoalgebra $C$ and a super-linear map $\pi: C \rightarrow \mathrm{U}$ such that $\pi\left(1_{C}\right)=0$. The connected super-coalgebras on $\mathrm{U}$ form a category; morphisms are supercoalgebra maps on $\mathrm{U}$, by which we mean super-coalgebra maps compatible with the maps to U. A terminal object is said to be cofree; see [21, Chapter $\mathrm{XII}$.

Proposition 3.11. There exists uniquely, up to isomorphism, a cofree connected super-coalgebra, $(\mathfrak{B}(\mathrm{U}), \pi)$, on $\mathrm{U}$.

Proof. The uniqueness is obvious. To prove the existence, suppose $\mathrm{U}=$ $\mathrm{U}^{\prime} \oplus \mathrm{U}^{\prime \prime}$, and that $\left(\mathfrak{B}\left(\mathrm{U}^{\prime}\right), \pi^{\prime}\right)$ and $\left(\mathfrak{B}\left(\mathrm{U}^{\prime \prime}\right), \pi^{\prime \prime}\right)$ are found. Then one sees that $\left(\mathfrak{B}\left(\mathrm{U}^{\prime}\right) \otimes \mathfrak{B}\left(\mathrm{U}^{\prime \prime}\right), \pi^{\prime} \otimes \epsilon+\epsilon \otimes \pi^{\prime \prime}\right)$ is a cofree connected super-coalgebra on $\mathrm{U}$. Therefore, it suffices to prove the existence for $\mathrm{U}_{0}$ and $\mathrm{U}_{1}$.

As for $\mathrm{U}_{0}$, note that given a super-coalgebra $C, C_{1}$ is a super-coideal of $C$, and every super-coalgebra map from $C$ to an ordinary coalgebra uniquely 
factors through the quotient coalgebra $C_{0}=C / C_{1}$. Then we see that $(C, \pi) \mapsto\left(C_{0},\left.\pi\right|_{C_{0}}\right)$ gives a functor from the category of connected supercoalgebras on $\mathrm{U}_{0}$ to the full subcategory consisting of all purely even objects, which is left adjoint to the inclusion functor. Therefore, the terminal object $\left(\mathfrak{B}\left(\mathrm{U}_{0}\right), \pi\right)$ of the full subcategory, which exists by [21, Theorem 12.2.5], is the desired object.

As for $U_{1}$, the exterior algebra $\wedge\left(U_{1}\right)$, regarded as a hyper-super-algebra as in Example 3.10, and the natural projection $\pi: \wedge\left(\mathrm{U}_{1}\right) \rightarrow \wedge^{1}\left(\mathrm{U}_{1}\right)=\mathrm{U}_{1}$ give the desired object. Given a connected super-coalgebra $(C, \pi)$ on $\mathrm{U}_{1}$, the composites

$$
C \stackrel{\Delta_{n-1}}{\longrightarrow} C^{\otimes n} \stackrel{\pi^{\otimes n}}{\longrightarrow} \mathrm{U}_{1}^{\otimes n} \stackrel{\text { cano }}{\longrightarrow} \wedge^{n}\left(\mathrm{U}_{1}\right), \quad n>0,
$$

and $\epsilon: C \rightarrow \mathbb{k}=\wedge^{0}\left(\mathrm{U}_{1}\right)$ amount to the unique super-coalgebra map $C \rightarrow$ $\wedge\left(\mathrm{U}_{1}\right)$ on $\mathrm{U}_{1}$. This is shown by modifying the proof of the last cited theorem; one can suppose $\operatorname{dim} U_{1}<\infty$, and reduce to the fact that the dual connected super-algebra $\wedge\left(U_{1}^{*}\right)\left(=\wedge\left(U_{1}\right)^{*}\right)$ is universal among those connected superalgebras each of which is equipped with a super-linear map from $U_{1}^{*}$ to the maximal super-ideal; see also Proposition 3.14 below.

As is just proved, we have

$$
\mathfrak{B}(\mathrm{U})=\mathfrak{B}\left(\mathrm{U}_{0}\right) \otimes \wedge\left(\mathrm{U}_{1}\right) .
$$

Recall from [21, Section 12.3] that $\mathfrak{B}\left(\mathrm{U}_{0}\right)$ includes $\mathrm{U}_{0}$ so that $P\left(\mathfrak{B}\left(\mathrm{U}_{0}\right)\right)=\mathrm{U}_{0}$ and $\left.\pi\right|_{\cup_{0}}=\operatorname{id}_{\cup_{0}}$. Moreover, $\mathfrak{B}\left(\mathrm{U}_{0}\right)$ is a commutative hyper-algebra which has as its product, the unique super-coalgebra map $\mu: \mathfrak{B}\left(\mathrm{U}_{0}\right) \otimes \mathfrak{B}\left(\mathrm{U}_{0}\right) \rightarrow$ $\mathfrak{B}\left(\mathrm{U}_{0}\right)$ that makes the diagram

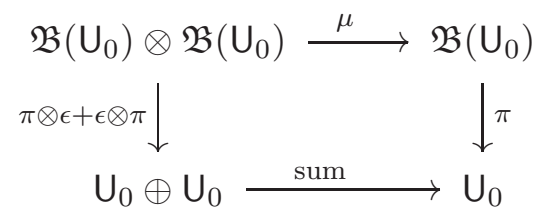

commutative. Given $0 \neq b \in \mathrm{U}_{0}, \mathfrak{B}\left(\mathrm{U}_{0}\right)$ includes uniquely a hyper-subalgebra $\mathfrak{B}_{b}$ which has a basis $b_{0}=1, b_{1}=b, b_{2}, b_{3}, \ldots$ of infinite length, such that $\pi\left(b_{r}\right)=\delta_{1, r} b$ and

$$
\Delta\left(b_{r}\right)=\sum_{k=0}^{r} b_{k} \otimes b_{r-k}, \quad \epsilon\left(b_{r}\right)=\delta_{0, r}, \quad b_{r} b_{s}=\left(\begin{array}{c}
r+s \\
r
\end{array}\right) b_{r+s},
$$

where $r, s \geq 0$. Given a basis $\left(b^{(i)}\right)_{i \in I}$ of $\mathbf{U}_{0}, \mathfrak{B}\left(\mathrm{U}_{0}\right)$ is the tensor product of $\mathfrak{B}_{b^{(i)}}, i \in I$.

With $\wedge\left(\mathrm{U}_{1}\right)$ as in Example 3.10, $\mathfrak{B}(\mathrm{U})=\mathfrak{B}\left(\mathrm{U}_{0}\right) \otimes \wedge\left(\mathrm{U}_{1}\right)$ is a supercommutative hyper-super-algebra. The product $\mathfrak{B}(U) \otimes \mathfrak{B}(U) \rightarrow \mathfrak{B}(U)$ on $\mathfrak{B}(\mathrm{U})$ gives a commutative diagram analogous to the last one. If $\operatorname{dim} \mathrm{U}_{0}=$ $m<\infty$ and $\operatorname{dim} \mathrm{U}_{1}=n<\infty$, then one obtains from the description $\mathfrak{B}\left(\mathrm{U}_{0}\right)=\bigotimes_{i} \mathfrak{B}_{b^{(i)}}$ above and (3.5), an isomorphism of super-algebras

$$
\mathfrak{B}(\mathrm{U})^{*} \simeq \mathbb{k}\left[\left[\mathrm{T}_{1}, \ldots, \mathrm{T}_{m+n}\right]\right],
$$

where the variables $\mathrm{T}_{1}, \ldots \mathrm{T}_{m}$ are even, and $\mathrm{T}_{m+1}, \ldots, \mathrm{T}_{m+n}$ odd. 
Let $C$ be a connected super-coalgebra, and set $\mathrm{U}=P(C)$. Choose a super-linear map $\pi: C \rightarrow \mathrm{U}$ such that $\pi\left(1_{C}\right)=0$ and $\left.\pi\right|_{\mathrm{U}}=\mathrm{id} \mathrm{U}$. We then have a unique super-coalgebra map

$$
f: C \rightarrow \mathfrak{B}(\mathrm{U})
$$

on $\mathrm{U}$. This is injective by [21, Lemma 11.0.1], since it is identical on $P(C)$.

Proposition 3.12. $C$ is smooth if and only if $f$ is an isomorphism.

Proof. If $C$ is smooth, then $f$ has a retraction, which is necessarily injective again by [21, Lemma 11.0.1]. Therefore, $f$ is an isomorphism.

Conversely, suppose $C=\mathfrak{B}(\mathrm{U})$. To see that $C$ is smooth, it suffices to prove the following, as is seen by a familiar argument using push-outs: an inclusion $C \hookrightarrow D$ into a super-cocommutative super-coalgebra $D$ such that $C \supset \operatorname{Corad} D$, or $D$ is connected, necessarily splits. To regard $D$ as a connected super-coalgebra on $\mathrm{U}$, extend the super-linear map $\pi: C=$ $\mathfrak{B}(\mathrm{U}) \rightarrow \mathrm{U}$ to $D \rightarrow \mathrm{U}$. Then the unique super-coalgebra map $D \rightarrow C$ on $\mathrm{U}$ is seen to be the desired retraction.

Let $C$ be a hyper-super-algebra. The pull-back of $C_{0} \otimes C_{0}$ along $\Delta$

$$
\underline{C}=\Delta^{-1}\left(C_{0} \otimes C_{0}\right)
$$

is the largest purely even super-subcoalgebra of $C$, and is in fact a Hopf subalgebra; see [10, p.291]. This $\underline{C}$ is now a hyper-algebra. Let $\mathfrak{g}=P(C)$. Then $\mathfrak{g}_{0}=P(\underline{C})$. Choose arbitrarily a totally ordered basis $\left(v_{i}\right)_{i \in I}$ of $\mathfrak{g}_{1}$. Then in $C$, the products $v_{i_{1}} \ldots v_{i_{n}}$ of increasing basis elements $v_{i_{1}}<\cdots<v_{i_{n}}$ of arbitrary length $n \geq 0$ form a left $\underline{C}$-free basis. Hence we have the unitpreserving isomorphism of left $\underline{C}$-super-module super-coalgebras

$$
\underline{C} \otimes \wedge\left(\mathfrak{g}_{1}\right) \stackrel{\simeq}{\longrightarrow} C
$$

which sends $v_{i_{1}} \wedge \cdots \wedge v_{i_{n}}$ in $\wedge\left(\mathfrak{g}_{1}\right)$ to the product $v_{i_{1}} \ldots v_{i_{n}}$ above; see [10, Theorem 3.6].

Proposition 3.13. For a hyper-super-algebra $C$, the following are equivalent:

(a) $C$ is smooth as a super-commutative super-coalgebra;

(b) $\underline{C}$ is smooth as a cocommutative coalgebra;

(c) $\underline{C}$ is isomorphic to $\mathfrak{B}\left(\mathfrak{g}_{0}\right)$ as a coalgebra.

If char $\mathbb{k}=0$, then we have

$$
C=\mathfrak{U}(\mathfrak{g}), \quad \underline{C}=\mathfrak{U}\left(\mathfrak{g}_{0}\right),
$$

and the equivalent conditions above are necessarily satisfied.

Proof. The last assertion in characteristic zero follows from Kostant's Theorem [21, Theorem 13.0.1] and the PBW Theorem; note that if $0 \neq x \in \mathfrak{g}_{0}$, the sequence $1, x, \ldots, x^{n} / n !, \ldots$ in $\mathfrak{U}\left(\mathfrak{g}_{0}\right)$ satisfies the first two equalities of (3.6).

(a) $\Rightarrow(b)$. This follows from the definition of smoothness since a supercoalgebra map from an ordinary coalgebra to $C$ takes values in $\underline{C}$.

(b) $\Leftrightarrow(\mathrm{c})$. This follows by Proposition 3.12 applied in the non-super situation. See also [22, Theorem 1.8.1, p.48] for a more generalized result. 
(c) $\Rightarrow$ (a). Suppose $\underline{C} \simeq \mathfrak{B}\left(\mathfrak{g}_{0}\right)$. Then by (3.10) we have an isomorphism $C \simeq \mathfrak{B}\left(\mathfrak{g}_{0}\right) \otimes \wedge\left(\mathfrak{g}_{1}\right)=\mathfrak{B}(\mathfrak{g})$ of connected super-coalgebras, which may be supposed to be an isomorphism on $\mathfrak{g}$. Proposition 3.12 ensures (a).

3.5. Duality theorem. Let $C$ be a connected super-coalgebra of finite type, and let $\mathbb{k} 1_{C}=C_{(0)} \subset C_{(1)} \subset \ldots$ be the coradical filtration on it. Set $\mathrm{U}=P(C)$, and choose a super-coalgebra injection $f: C \rightarrow \mathfrak{B}(\mathrm{U})=$ $\mathfrak{B}\left(\mathrm{U}_{0}\right) \otimes \wedge\left(\mathrm{U}_{1}\right)$ on $\mathrm{U}$ as in (3.8). Let $A=C^{*}$ denote the dual super-algebra. By definition of $C_{(n)}$ (see (3.2) ), the restriction maps $A=C^{*} \rightarrow\left(C_{(n)}\right)^{*}$ induce isomorphisms

$$
A / \mathfrak{m}^{n+1} \simeq\left(C_{(n)}\right)^{*}, \quad n>0,
$$

where $\mathfrak{m}$ denotes the kernel of $A \rightarrow\left(C_{(0)}\right)^{*}=\mathbb{k}$. Therefore, we have $A \simeq \lim _{\longleftarrow n} A / \mathfrak{m}^{n}$, whence $A$ is a complete connected super-algebra with the maximal $\mathfrak{m}$. Since $\left(C_{(1)} / \mathbb{k} 1_{C}\right)^{*} \simeq \mathfrak{m} / \mathfrak{m}^{2}$, it follows from (3.7) that $f$ is dualized to a super-algebra surjection

$$
\mathbb{k}\left[\left[\mathrm{T}_{1}, \ldots, \mathrm{T}_{m+n}\right]\right] \rightarrow A,
$$

which maps $\mathrm{T}_{1}, \ldots, \mathrm{T}_{m+n}$ to a basis of $\mathfrak{m}$ modulo $\mathfrak{m}^{2}$. Therefore, $A$ is Noetherian. It follows from Propositions 3.2 and 3.12 that the surjection is an isomorphism, if and only if $A$ is smooth, if and only if $C$ is smooth.

Let $A$ be a Noetherian complete connected super-algebra. Since $\mathfrak{m}_{A}$ is then finitely generated, $\mathfrak{m}_{A}^{n} / \mathfrak{m}_{A}^{n+1}, n \geq 0$, are all finite-dimensional. Therefore, $A / \mathfrak{m}_{A}^{n+1}, n \geq 0$, are finite-dimensional super-algebras. Let $C_{(n)}=$ $\left(A / \mathfrak{m}_{A}^{n+1}\right)^{*}$ be the dual super-coalgebras. One sees that $\operatorname{dim} C_{(0)}=1$, and $C_{(0)} \subset C_{(1)} \subset \ldots$ in $A^{*}$. We have the filtered union

$$
A^{\star}=\bigcup_{n \geq 0} C_{(n)}
$$

of the super-coalgebras. This $A^{\star}$, consisting of all continuous linear maps $A \rightarrow \mathbb{k}$, may be called the continuous dual of $A$. The composite

$$
A \rightarrow\left(A^{\star}\right)^{*} \simeq \lim _{\longleftarrow n}\left(C_{(n)}\right)^{*} \simeq \lim _{\longleftarrow n} A / \mathfrak{m}_{A}^{n}
$$

of the canonical map $A \rightarrow\left(A^{\star}\right)^{*}$ with natural isomorphisms coincides with the canonical isomorphism $A \simeq \lim _{\longleftarrow n} A / \mathfrak{m}_{A}^{n}$. Therefore, the canonical $A \rightarrow$ $\left(A^{\star}\right)^{*}$ is an isomorphism. The sequence $C_{(n)} \hookrightarrow A^{\star} \rightarrow\left(A^{\star} / C_{(0)}\right)^{\otimes(n+1)}$ given by the iterated coproduct is exact, since it is dualized to the exact sequence $A / \mathfrak{m}_{A}^{n+1} \leftarrow A \leftarrow \mathfrak{m}_{A}^{\hat{\otimes}(n+1)}$ given by the iterated product. Therefore, $C_{(0)} \subset C_{(1)} \subset \ldots$ is the coradical filtration on $A^{\star}$, whence $A^{\star}$ is a connected super-coalgebra of finite type.

One sees that the assignments $C \mapsto C^{*}$ and $A \mapsto A^{\star}$ above give rise to contra-variant functors. Moreover, we have the following.

Proposition 3.14. $C \mapsto C^{*}$ and $A \mapsto A^{\star}$ give an anti-equivalence between

- the category of Noetherian complete connected super-algebras, and

- the category of connected super-coalgebras of finite type.

This restricts to an anti-equivalence between the full subcategories consisting of the smooth objects. 
Proof. Since we have seen that given an $A$, we have a natural isomorphism $A \simeq\left(A^{\star}\right)^{*}$, it remains to prove that given a $C$, we have a natural isomorphism $C \simeq\left(C^{*}\right)^{\star}$; recall we have seen that $C$ is smooth if and only if $C^{*}$ is. Indeed, the isomorphisms $C_{(n)} \simeq\left(A / \mathfrak{m}^{n+1}\right)^{*}, n>0$, dual to (3.11) amount to a desired isomorphism.

Theorem 3.15. The anti-equivalence above induces an anti-equivalence between

- the category of (smooth) Noetherian complete Hopf super-algebras $A$ and

- the category of (smooth) hyper-super-algebras $C$ of finite type.

Proof. Suppose that we are in the situation of Proposition 3.14. In the former (resp., latter) category, objects are closed under the coproduct $\hat{\otimes}$ (resp., the product $\otimes)$. The proved anti-equivalence induces an anti-equivalence between the co-group objects and the group objects; this proves the theorem.

Note that $C \mapsto \underline{C}$ gives a functor from the category of hyper-superalgebras (of finite type) to the category of hyper-algebras (of finite type).

Corollary 3.16. We have the following.

(1) If $A$ is a Noetherian complete Hopf super-algebra, then $\bar{A}$ is naturally a Noetherian complete Hopf algebra; $A$ is smooth if and only if $\bar{A}$ is. The construction gives rise to a functor $A \mapsto \bar{A}$.

(2) $A \mapsto \bar{A}$ and $C \mapsto \underline{C}$ are compatible with the anti-equivalence of Theorem 3.15 and the restricted one between the purely even objects. To be more explicit, if $A=C^{*}$ or $A^{\star}=C$, then $\bar{A}=\underline{C}^{*}$ or $\bar{A}^{\star}=\underline{C}$.

Proof. (1) By Theorem 3.15 we have $A=C^{*}$, where $C$ is a hyper-superalgebra of finite type. We see from (3.10) that $\bar{A}=\underline{C}^{*}$, and this $\bar{A}$ is a Noetherian complete Hopf algebra such that the canonical $A \rightarrow \bar{A}$ preserves the coproduct. The assertion on smoothness follows from Propositions 3.13 and 3.14. The functoriality is easy to see.

(2) This is seen from the argument above.

Corollary 3.17. Assume char $\mathbb{k}=0$. Then every Noetherian complete Hopf super-algebra is smooth, and we have a natural anti-equivalence between

- the category of Noetherian complete Hopf super-algebras and

- the category of finite-dimensional Lie super-algebras.

Proof. This follows from Theorem 3.15, since if char $\mathbb{k}=0$, then $\mathfrak{g} \mapsto \mathfrak{U}(\mathfrak{g})$ gives an equivalence from the category of (finite-dimensional) Lie superalgebras to the category of hyper-super-algebras (of finite-type), and hence every hyper-super-algebra is smooth; see Proposition 3.13.

Let $A$ be a Noetherian complete Hopf super-algebra, and let $C=A^{\star}$ be the corresponding hyper-super-algebra; Note that the complete topological right $A$-super-comodules and the complete topological left $C$-super-modules (see Proposition 2.3 for definition) respectively form symmetric tensor categories; for both the tensor product is given by $\hat{\otimes}$, and the unit object is the discrete $\mathbb{k}$ equipped with the trivial structure. 
Proposition 3.18. The two symmetric tensor categories are isomorphic to each other.

Proof. This follows from Proposition 2.3 for $R$ applied to the present $C$.

\section{SUPER-MANIFOLDS}

In what follows, $\mathbb{k}$ is supposed to be a complete field (see Section 1.1) of characteristic $\neq 2$, unless otherwise stated.

4.1. Convergent power series super-algebra. Given non-negative integers $m$ and $n$, the convergent power series super-algebra

$$
\mathbb{k}\left\{\mathrm{T}_{1}, \ldots, \mathrm{T}_{m+n}\right\}
$$

in $m$ even and $n$ odd variables is the tensor product

$$
\mathbb{k}\left\{\mathrm{T}_{1}, \ldots, \mathrm{T}_{m}\right\} \otimes \wedge\left(\mathrm{T}_{m+1}, \ldots, \mathrm{T}_{m+n}\right)
$$

of the convergent power series algebra $\mathbb{k}\left\{\mathrm{T}_{1}, \ldots, \mathrm{T}_{m}\right\}$ and the exterior algebra $\wedge\left(\mathrm{T}_{m+1}, \ldots, \mathrm{T}_{m+n}\right)$ as in (3.1). This is a smooth (or geometrically regular [16, Theorem A.2]) Noetherian connected super-algebra, whose completion is the formal power series super-algebra $\mathbb{k}\left[\left[\mathrm{T}_{1}, \ldots, \mathrm{T}_{m+n}\right]\right]$. Note that the variables $\mathrm{T}_{1}, \ldots, \mathrm{T}_{m+n}$ give a basis of the maximal super-ideal $\mathfrak{m}$ of $\mathbb{k}\left\{\mathrm{T}_{1}, \ldots \mathrm{T}_{m+n}\right\}$ modulo $\mathfrak{m}^{2}$.

4.2. Super-manifolds. By a manifold we mean an analytic manifold $X$ over $\mathbb{k}$ as defined in [18, Part II, Chapter III, Section 2]. Thus a chart on $X$ is a triple $(U, \psi, n)$ consisting of an open sub-set $U$, a non-negative integer $n$ and a homeomorphism $\psi: U \rightarrow \psi(U)$ onto an open set of $\mathbb{k}^{n}$, where $n$ is not required to be constant. In addition, the underlying topological space $|X|$ of $X$ is not assumed to be Hausdorff or second countable.

Let $X$ be a manifold. We let $\mathcal{F}=\mathcal{F}_{X}$ denote the associated sheaf of analytic functions. Let $\mathrm{W}$ be a finite-dimensional vector space. We let

$$
\mathcal{F} \otimes \wedge(\mathrm{W})
$$

denote the sheaf of super-algebras on $|X|$ which associates $\mathcal{F}(U) \otimes \wedge(\mathrm{W})$ to every open set $U$.

Definition 4.1. (1) A super-ringed space is a pair $X=\left(|X|, \mathcal{O}_{X}\right)$ of a topological space $|X|$ and a sheaf $\mathcal{O}_{X}$ of super-algebras on $|X|$; by convention, $\mathcal{O}(\emptyset)=0$. We will often write $\mathcal{O}$ for $\mathcal{O}_{X}$. The super-ringed spaces form a category SRS. A morphism $\phi=\left(|\phi|, \phi^{*}\right): X \rightarrow Y$ in SRS consists of a continuous map $|\phi|:|X| \rightarrow|Y|$ and a sheaf-morphism $\phi^{*}: \mathcal{O}_{Y} \rightarrow \phi_{*} \mathcal{O}_{X}$ of super-algebras.

(2) The pair $(|X|, \mathcal{F} \otimes \wedge(\mathrm{W}))$ obtained as above from a manifold $X$ is a super-ringed space. A super-ringed space of this form is called a strongly split super-manifold, or an s-split super-manifold, in short; compare with the notion of split super-manifold (see [25, for example), whose structure sheaf is only required to be the exterior algebra on some locally free $\mathcal{F}$ module. Let SSMFD denote the full subcategory of SRS which consists of all s-split super-manifolds; it includes the category MFD of manifolds as a full subcategory. 
(3) A super-manifold is a super-ringed space $X=(|X|, \mathcal{O})$ which is locally isomorphic to an s-split super-manifold, or namely, which has an open covering $|X|=\bigcup_{i \in I} U_{i}$ such that each $\left(U_{i},\left.\mathcal{O}\right|_{U_{i}}\right)$ is isomorphic to an s-split super-manifold. For a non-empty open set $U$ of $|X|,\left(U,\left.\mathcal{O}\right|_{U}\right)$ is obviously a super-manifold, and such is called an open super-submanifold of $X$. Let SMFD denote the full subcategory SRS which consists of all super-manifolds; it includes SSMFD and MFD as full subcategories.

We thus have the chain of categories

$$
\mathrm{MFD} \subset \mathrm{SSMFD} \subset \mathrm{SMFD} \subset \mathrm{SRS} .
$$

The category SRS has the terminal object $(\{*\}, \mathbb{k})$, the one-point set $\{*\}$ equipped with the sheaf $\{*\} \mapsto \mathbb{k}$. Contained in MFD, this is necessarily a terminal object of each of the rest.

Given a super-manifold $X=(|X|, \mathcal{O})$, let $\mathcal{O}_{\text {red }}$ denote the sheafification of the presheaf on $|X|$ which associates $\mathcal{O}(U)_{\text {red }}(=\overline{\mathcal{O}(U)})$ to an open set $U$; see (2.3). If $U$ is included in an open set $V$ such that $\left(V,\left.\mathcal{O}\right|_{V}\right)$ is an s-split super-manifold, then $\mathcal{O}_{\text {red }}(U)=\mathcal{O}(U)_{\text {red }}$. One sees that $\left(|X|, \mathcal{O}_{\text {red }}\right)$ is a manifold, which will be presented as

$$
X_{\text {red }}=\left(|X|, \mathcal{O}_{\text {red }}\right) .
$$

We see easily the following.

Lemma 4.2. $X \mapsto X_{\text {red }}$ gives rise to a functor

$$
\text { ( ) } \text { red }: \text { SMFD } \rightarrow \text { MFD, }
$$

which, restricted to MFD, is the identity functor. The functor is right adjoint to the inclusion functor MFD $\rightarrow$ SMFD, or explicitly, we have

$$
\operatorname{Mor}(Y, X)=\operatorname{Mor}\left(Y, X_{\text {red }}\right)
$$

if $Y \in$ MFD.

Here and in what follows we let $\operatorname{Mor}(Y, X)$ denote the set of all morphisms $Y \rightarrow X$ in SMFD.

Let $X=(|X|, \mathcal{O})$ be a super-manifold, and let $x \in|X|$. If one chooses an s-split open super-submanifold $(U, \mathcal{F} \otimes \wedge(\mathrm{W}))$ of $X$ such that $x \in U$, then the stalk $\mathcal{O}_{x}$ at $x$ is identified with $\mathcal{F}_{x} \otimes \wedge(\mathrm{W})$, which is isomorphic to the convergent power series super-algebra $\mathbb{k}\left\{\mathrm{T}_{1}, \ldots, \mathrm{T}_{m+n}\right\}$ in $m$ even and $n$ odd variables (see (4.1)), where

$$
m=\operatorname{Kdim} \mathcal{F}_{x}, \quad n=\operatorname{dim} \mathbf{W}
$$

Kdim indicates the Krull dimension. One sees easily

$$
\begin{aligned}
& \left(\mathcal{O}_{x}\right)_{\text {red }}=\left(\mathcal{O}_{\text {red }}\right)_{x} \simeq \mathbb{k}\left\{\mathrm{T}_{1}, \ldots, \mathbf{T}_{m}\right\}, \\
& \left(\hat{\mathcal{O}}_{x}\right)_{\text {red }}=\left(\hat{\mathcal{O}}_{\text {red }}\right)_{x} \simeq \mathbb{k}\left[\left[\mathrm{T}_{1}, \ldots, \mathbf{T}_{m}\right]\right] .
\end{aligned}
$$

Let $\mathfrak{m}_{x}\left(\right.$ or $\left.\mathfrak{m}_{X, x}\right)$ denote the maximal super-ideal of $\mathcal{O}_{x}$. The tangent superspace $T_{x} X$ of $X$ at $x$ is the dual super-vector space

$$
T_{x} X=\left(\mathfrak{m}_{x} / \mathfrak{m}_{x}^{2}\right)^{*}
$$

of $\mathfrak{m}_{x} / \mathfrak{m}_{x}^{2}$, the cotangent super-vector space. We have

$$
m=\operatorname{dim}\left(T_{x} X\right)_{0}, \quad n=\operatorname{dim}\left(T_{x} X\right)_{1} .
$$


Definition 4.3. We present as

$$
m \mid n=\operatorname{sdim}_{x} X,
$$

and call this the super-dimension of $X$ at $x$.

One sees that $x \mapsto \operatorname{sdim}_{x} X$ is locally constant; see [18, Part II, Chapter 3 , Section 2]. We have

$$
\left(T_{x} X\right)_{0}=T_{x}\left(X_{\text {red }}\right)
$$

and so the dimension $m$ equals the dimension $\operatorname{dim}_{x}\left(X_{\text {red }}\right)$ of the manifold $X_{\text {red }}$ at $x$, as defined in [18.

We see easily the following.

Lemma 4.4. A super-manifold $X=(|X|, \mathcal{O})$ is s-split if and only if $\mathcal{O} \simeq$ $\mathcal{O}_{\text {red }} \otimes \wedge(\mathrm{W})$, where $\mathrm{W}=\left(T_{x} X\right)_{1}^{*}$ for some/any point $x \in|X|$.

If these equivalent conditions are satisfied, we will often write $\mathcal{F} \otimes \wedge(\mathrm{W})$ for $\mathcal{O}=\mathcal{O}_{X}$ (as we have done so far), supposing $\mathcal{F}=\mathcal{F}_{X_{\text {red }}}\left(=\mathcal{O}_{\text {red }}\right)$.

Let $\phi: X \rightarrow Y$ be a morphism of super-manifolds. Let $x \in|X|$, and set $y=|\phi|(x)$. The sheaf-morphism $\phi^{*}: \mathcal{O}_{Y} \rightarrow \phi_{*} \mathcal{O}_{X}$ induces a super-algebra map $\phi_{x}^{*}: \mathcal{O}_{Y, y} \rightarrow \mathcal{O}_{X, x}$. This $\phi_{x}^{*}$ is necessarily local in the sense $\phi_{x}^{*}\left(\mathfrak{m}_{Y, y}\right) \subset$ $\mathfrak{m}_{X, x}$. Therefore, it induces a super-linear map $\mathfrak{m}_{Y, y} / \mathfrak{m}_{Y, y}^{2} \rightarrow \mathfrak{m}_{X, x} / \mathfrak{m}_{X, x}^{2}$. The dual super-linear map is denoted by

$$
d \phi_{x}: T_{x} X \rightarrow T_{y} Y .
$$

The following is the Inverse Function Theorem for super-manifolds; cf 1 , Proposition 5.1.1]. This basic result will not be used in what follows, but may be worth to record here. In addition, the same argument as proving the theorem will be used to prove Lemma [5.9, which will be used at the final step of proving Theorem 6.3.

Theorem 4.5. Let $\phi: X \rightarrow Y$ be a morphism of super-manifolds. Let $x \in$ $|X|$, and set $y=|\phi|(x)$. Assume that $d \phi_{x}: T_{x} X \rightarrow T_{y} Y$ is an isomorphism. Then there exist open neighborhoods $U$ of $x$ and $V$ of $y$, such that $\phi$ restricts to an isomorphism $U \rightarrow V$ of super-manifolds.

Proof. Note that $\left(d \phi_{x}\right)_{0}$ coincides with $d\left(\phi_{\text {red }}\right)_{x}: T_{x}\left(X_{\text {red }}\right) \rightarrow T_{y}\left(Y_{\text {red }}\right)$. The Inverse Function Theorem [18, Theorem 2, Page 83] for manifolds, applied to this now isomorphism, allows us to suppose, replacing $|X|$ and $|Y|$ with some open sub-sets, that $\phi_{\text {red }}: X_{\text {red }} \rightarrow Y_{\text {red }}$ is an isomorphism of manifolds and, moreover, $X$ and $Y$ are s-split so that

$$
\mathcal{O}_{X}=\mathcal{F}_{X} \otimes \wedge\left(\mathrm{W}_{X}\right), \quad \mathcal{O}_{Y}=\mathcal{F}_{Y} \otimes \wedge\left(\mathrm{W}_{Y}\right) .
$$

(For simplicity we write $\mathcal{F}_{X}, \mathcal{F}_{Y}$ as above, which should be denoted by $\mathcal{F}_{X_{\text {red }}}, \mathcal{F}_{Y_{\text {red }}}$, to be precise; see the paragraph following Lemma 4.4.)

We wish to prove the sheaf-morphism $\phi^{*}: \mathcal{O}_{Y} \rightarrow \phi_{*} \mathcal{O}_{X}$, restricted to $V=|\phi|(U)$, is an isomorphism, where $U \subset|X|$ is some open neighborhood of $x$. This is equivalent to proving that the map $\phi_{z}^{*}: \mathcal{O}_{Y, w} \rightarrow \mathcal{O}_{X, z}$ of connected super-algebras is an isomorphism for each $z \in U$ with $w=|\phi|(z)$. Applying the functor gr given in Section 2.3, we have an $\mathbb{N}$-graded algebra map $\operatorname{gr}\left(\phi_{z}^{*}\right): \mathcal{F}_{Y, w} \otimes \wedge\left(\mathrm{W}_{Y}\right) \rightarrow \mathcal{F}_{X, z} \otimes \wedge\left(\mathrm{W}_{X}\right)$; note that we are in the special situation as noted in Remark 2.2. We see that $\phi_{z}^{*}$ is isomorphic if and only 
if $\operatorname{gr}\left(\phi_{z}^{*}\right)$ is isomorphic if and only if the maps $\operatorname{gr}\left(\phi_{z}^{*}\right)(0)$ and $\operatorname{gr}\left(\phi_{z}^{*}\right)(1)$ in degree 0 and 1 are isomorphic. The first map in degree 0 is identified with $\left(\phi_{\text {red }}\right)_{z}^{*}$, and is, therefore, isomorphic.

Let us turn to the second map $\operatorname{gr}\left(\phi_{z}^{*}\right)(1): \mathcal{F}_{Y, w} \otimes \mathrm{W}_{Y} \rightarrow \mathcal{F}_{X, z} \otimes \mathrm{W}_{X}$ in degree 1 . This is regarded as a morphism of finitely generated free modules over a connected algebra, when we identify the connected algebra $\mathcal{F}_{Y, w}$ with $\mathcal{F}_{X, z}$ via $\operatorname{gr}\left(\phi_{z}^{*}\right)(0)$. When $z=x$, this $\operatorname{gr}\left(\phi_{x}^{*}\right)(1)$ is isomorphic, since the map modulo the maximal ideal is dual to the isomorphism $\left(d \phi_{x}\right)_{1}$, and is, therefore, isomorphic. In particular, it follows that $\operatorname{dim} \mathrm{W}_{X}=\operatorname{dim} \mathrm{W}_{Y}$; we denote this dimension by $n$.

Associated with the $\phi^{*}$ above is a sheaf-morphism $\operatorname{gr}\left(\phi^{*}\right): \mathcal{F}_{Y} \otimes \wedge\left(\mathrm{W}_{Y}\right) \rightarrow$ $\left(\phi_{\text {red }}\right)_{*} \mathcal{F}_{X} \otimes \wedge\left(\mathrm{W}_{X}\right)$ of (super-commutative) $\mathbb{N}$-graded algebras. Passing to stalks we have the $\mathbb{N}$-graded algebra maps $\operatorname{gr}\left(\phi^{*}\right)_{z}, z \in|X|$. They coincide with the $\operatorname{gr}\left(\phi_{z}^{*}\right)$ before, since in the present situation, taking gr commutes with the construction of stalks. The morphism $\operatorname{gr}\left(\phi^{*}\right)(0)$ in degree 0 , coinciding with $\left(\phi_{\text {red }}\right)^{*}$, is isomorphic. We suppose that $\left|\phi_{\text {red }}\right|$ is the identity map, and identify the two sheaves so that $\mathcal{F}_{X}=\left(\phi_{\text {red }}\right)_{*} \mathcal{F}_{X}=\mathcal{F}_{Y}$. By choosing bases of $\mathrm{W}_{X}$ and of $\mathrm{W}_{Y}$, the morphism $\operatorname{gr}\left(\phi^{*}\right)(1): \mathcal{F}_{Y} \otimes \mathrm{W}_{Y} \rightarrow \mathcal{F}_{X} \otimes \mathrm{W}_{X}$ in degree 1 is presented by an $n \times n$ matrix with entries in $\mathcal{F}_{X}$. Let $\operatorname{det} \in \mathcal{F}_{X}(|X|)$ denote the determinant of the matrix evaluated in $|X|$. Given $z \in|X|$, we see that $\operatorname{gr}\left(\phi_{z}^{*}\right)(1)$ is isomorphic if and only if the natural image of det in $\mathcal{F}_{X, z}$ is invertible if and only if the analytic function det : $|X| \rightarrow \mathbb{k}$ does not vanish at $z$. Since this is the case for $x$, there exists an open neighborhood $U$ of $x$ such that for every $z \in U$, the last condition and hence the equivalent other conditions are all satisfied. This completes the proof.

The following is crucial to define super Lie groups; see Definition 5.1 .

Proposition 4.6. The category SMFD of super-manifolds has finite products.

Proof. This follows, by modifying the proof of the result [3, Remark 2.6 (iv)] in the $C^{\infty}$ situation, so as to fit in with our analytic situation including the positive characteristic case. Let us give some details below.

The category contains the terminal object $(\{*\}, \mathbb{k})$, as was seen before.

It remains to show the existence of the product $X_{1} \times X_{2}$ of two supermanifolds $X_{1}, X_{2}$. One sees that the s-split super-manifold $(|X|, \mathcal{F} \otimes \wedge(\mathrm{W}))$ decomposes into the (categorical) product

$$
(|X|, \mathcal{F} \otimes \wedge(\mathrm{W}))=(|X|, \mathcal{F}) \times(\{*\}, \wedge(\mathrm{W})),
$$

where $Y:=(\{*\}, \wedge(\mathrm{W}))$ is the one-point set $\{*\}$ equipped with the sheaf $\{*\} \mapsto \wedge(\mathrm{W})$. Essential for this is to see that given a super-manifold $Z=(|Z|, \mathcal{O})$, the set $\operatorname{Mor}(Z, Y)$ of the morphisms is identified with the set $\operatorname{Hom}\left(\mathrm{W}, \mathcal{O}(|Z|)_{1}\right)$ of all linear maps from $W$ to the odd component of $\mathcal{O}(|Z|)$. This also shows that the product $\left(\{*\}, \wedge\left(\mathrm{W}_{1}\right)\right) \times\left(\{*\}, \wedge\left(\mathrm{W}_{2}\right)\right)$ is given by $\left(\{*\}, \wedge\left(\mathrm{W}_{1} \oplus \mathrm{W}_{2}\right)\right)$. Therefore, we may suppose that $X_{i}, i=1,2$, are manifolds, and are, moreover (by gluing), open sub-manifolds of some $\mathbb{k}^{m_{i}}$, and it suffices to show that their product $X_{1} \times X_{2}$ in MFD gives the desired product.

Suppose that $X$ is a non-empty open sub-manifold of $\mathbb{k}^{m}$, and let $Z$ be as above. The desired result follows from the following. 
Claim. $\operatorname{Mor}(Z, X)$ is in a natural one-to-one correspondence with the set

$$
\left\{\left(f_{1}, \ldots, f_{m}\right) \in \mathcal{O}(|Z|)_{0}^{m}\left|\left(\bar{f}_{1}(z), \ldots, \bar{f}_{m}(z)\right) \in\right| X \mid \text { for all } z \in|Z|\right\},
$$

where $\bar{f}_{i}$ denotes the natural image of $f_{i}$ in $\mathcal{O}_{\text {red }}(|Z|)$.

This is proved by [3, Proposition 2.4] in the $C^{\infty}$ situation. Choose $\left(f_{1}, \ldots, f_{m}\right)$ from the set above. Note from [3] that the main point of the proof of Claim is to construct the corresponding morphism $Z \rightarrow X$. Let us confirm only that the construction indeed works in our situation. We have a morphism of manifolds,

$$
\phi: Z_{\mathrm{red}} \rightarrow X, \quad \phi(z)=\left(\bar{f}_{1}(z), \ldots, \bar{f}_{m}(z)\right) .
$$

The associated sheaf-morphism $\phi^{*}: \mathcal{F} \rightarrow \phi_{*} \mathcal{O}_{\text {red }}$ is given by

$$
\phi^{*}: \mathcal{F}(U) \rightarrow \mathcal{O}_{\text {red }}(V), \quad \phi^{*}(F)=F\left(\bar{f}_{1}, \ldots, \bar{f}_{m}\right),
$$

where $U \subset|X|$ and $V \subset|Z|$ are open with $\phi(V) \subset U$. The desired morphism $\left(\phi, \widetilde{\phi^{*}}\right)$ consists of the same continuous map $\phi$ as above, and the sheafmorphism $\widetilde{\phi^{*}}: \mathcal{F} \rightarrow \phi_{*} \mathcal{O}$ given by

$$
\widetilde{\phi^{*}}: \mathcal{F}(U) \rightarrow \mathcal{O}(V), \quad \widetilde{\phi^{*}}(F)=F\left(f_{1}, \ldots, f_{m}\right)
$$

where $U$ and $V$ are as above. In (4.7), (4.8) and in what follows we wrote and will write $f_{i}, \bar{f}_{i}$ for $\left.f_{i}\right|_{V},\left.\bar{f}_{i}\right|_{V}$. Our task is to clarify what the composite $F\left(f_{1}, \ldots, f_{m}\right)$ in (4.8) is.

Now, we assume in addition that $V$ is s-split as a super-manifold, and proceed, depending on [18, Part II, Chaper II] for basic results and the notation which uses multi-indexes, $\boldsymbol{k}=\left(k_{1}, \ldots, k_{m}\right), k_{i} \geq 0$. We see from [18, Lemma on Page 70] the following.

Fact. For each $\boldsymbol{k}$, we have a unique operator $\Delta^{k}: \mathcal{F}(U) \rightarrow \mathcal{F}(U)$ that gives rise to the operator

$$
\Delta^{k}\left(\sum_{i} c_{i} T^{i}\right)=\sum_{i \geq k}\left(\begin{array}{l}
i \\
k
\end{array}\right) c_{i} T^{i-k}
$$

on $\mathcal{F}_{x}=\mathbb{k}\left\{\mathrm{T}_{1}, \ldots, \mathrm{T}_{m}\right\}$ at every $x \in U$, where $\mathrm{T}^{\boldsymbol{i}}=\mathrm{T}_{1}^{i_{1}} \ldots \mathrm{T}_{m}^{i_{m}}$ and $\left(\begin{array}{l}\boldsymbol{i} \\ j\end{array}\right)=$ $\left(\begin{array}{l}i_{1} \\ j_{1}\end{array}\right) \ldots\left(\begin{array}{l}i_{m} \\ j_{m}\end{array}\right)$; see Example 5.16 .

One may understand that the formula above defines $\Delta^{k} f$ locally, when $f \in \mathcal{F}(U)$ is expressed as a convergent power series in some strict polydisk. Since $V$ is s-split, we have the decomposition

$$
f_{i}=\bar{f}_{i}+g_{i}, \quad 1 \leq i \leq m,
$$

in $\mathcal{O}(V)$, where $g_{i} \in \mathcal{O}(V)$ are nilpotent even elements. Define $F\left(f_{1}, \ldots, f_{m}\right)$ by

$$
F\left(f_{1}, \ldots, f_{m}\right)=\sum_{\boldsymbol{k}} \Delta^{k} F\left(\bar{f}_{1}, \ldots, \bar{f}_{m}\right) g^{k},
$$

where $g^{k}=g_{1}^{k_{1}} \ldots g_{m}^{k_{m}}$. Note that this sum is finite. The formula given in Part 3 of the last cited [18, Lemma on Page 70] shows that $F\left(f_{1}, \ldots, f_{m}\right)$ is indeed the section over $V$ which assigns to every $z \in V$, the composite $F_{x}\left(\left(f_{1}\right)_{z}, \ldots,\left(f_{m}\right)_{z}\right) \in \mathcal{O}_{z}$, where $x=\phi(z)$. 
We see that the last assignment, being of local nature, defines a section without the assumption that $V$ is s-split; apply the result with the assumption to open subsets which are s-split as super-manifolds. In conclusion, $F\left(f_{1}, \ldots, f_{m}\right)$ is the section thus defined over any $V$ with $\phi(V) \subset U$.

Remark 4.7. Let $X$ and $Y$ be super-manifolds, and set $Z=X \times Y$.

(1) We have $Z_{\text {red }}=X_{\text {red }} \times Y_{\text {red }}$ in MFD, as is seen from (4.3). In particular, $|Z|$ is the product $|X| \times|Y|$ of topological spaces.

(2) Suppose that $X=\left(|X|, \mathcal{F}_{X_{\text {red }}} \otimes \wedge\left(\mathrm{W}_{X}\right)\right)$ and $Y=\left(|Y|, \mathcal{F}_{Y_{\text {red }}} \otimes \wedge\left(\mathrm{W}_{Y}\right)\right)$ are s-split. As is seen from the last proof, $Z$ is then s-split so that

$$
Z=\left(|X| \times|Y|, \mathcal{F}_{X_{\text {red }} \times Y_{\text {red }}} \otimes \wedge\left(\mathrm{W}_{X} \oplus \mathrm{W}_{Y}\right)\right) .
$$

In addition, the canonical morphism from $Z$ to $X$, for example, is the projection pr: $|X| \times|Y| \rightarrow|X|$ equipped with the tensor product of (i) the canonical sheaf-morphism $\mathcal{F}_{X_{\text {red }}} \rightarrow \operatorname{pr}_{*} \mathcal{F}_{X_{\text {red }} \times Y_{\text {red }}}$ and (ii) the super-algebra map $\wedge\left(\mathrm{W}_{X}\right) \rightarrow \wedge\left(\mathrm{W}_{X} \oplus \mathrm{W}_{Y}\right)$ induced from the inclusion $\mathrm{W}_{X} \hookrightarrow \mathrm{W}_{X} \oplus \mathrm{W}_{Y}$.

Proposition 4.8. Let $X$ and $Y$ be super-manifolds, and suppose $x \in|X|$ and $y \in|Y|$. The maps

$$
\hat{\mathcal{O}}_{X, x} \rightarrow \hat{\mathcal{O}}_{X \times Y,(x, y)} \leftarrow \hat{\mathcal{O}}_{Y, y}, \quad T_{x} X \leftarrow T_{(x, y)}(X \times Y) \rightarrow T_{y} Y
$$

of complete connected super-algebras, and of super-vector spaces that are induced from the canonical morphisms $X \leftarrow X \times Y \rightarrow Y$ naturally give rise to isomorphisms

$$
\hat{\mathcal{O}}_{X, x} \hat{\otimes} \hat{\mathcal{O}}_{Y, y} \simeq \hat{\mathcal{O}}_{X \times Y,(x, y)}, \quad T_{(x, y)}(X \times Y) \simeq T_{x} X \oplus T_{y} Y .
$$

Proof. We may suppose that $X$ and $Y$ are s-split. The desired result then follows easily from Remark 4.7 (2).

Through the isomorphisms (4.10) we will identify the relevant objects.

\section{Super LiE Groups}

5.1. Super Lie groups and their hyper-super-algebras. The following definition makes sense by Proposition 4.6 .

Definition 5.1. A super Lie group is a group object in SMFD. We let SLG denote the category of those group objects. See Remark 5.5 (2) for the definition of morphisms.

We will see that every super Lie group is s-split as a super manifold; see Corollary 6.13. The underlying topological space of a super Lie group is a group, in particular. The identity element will be denoted by $e$.

By Remark 4.7 (2) the functor ( $)_{\text {red }}:$ SMFD $\rightarrow$ MFD given by Lemma 4.2 preserves finite products, and so it induces a functor between the categories of the group objects. This proves the following.

Lemma 5.2. If $G=(|G|, \mathcal{O})$ is a super Lie group, then

$$
G_{\text {red }}=\left(|G|, \mathcal{O}_{\text {red }}\right)
$$

is a Lie group, that is, a group object in MFD. The assignment $G \mapsto G_{\mathrm{red}}$ gives rise to a functor from SLG to the category of Lie groups.

We see easily the following. 
Lemma 5.3. Let $G=(|G|, \mathcal{O})$ be a super Lie group.

(1) Let $g \in|G|$. The left multiplication by $g$

$$
L_{g}: G \rightarrow G, L_{g}(h)=g h
$$

is an isomorphism in SMFD, which induces isomorphisms

$$
\left(L_{g}^{*}\right)_{e}: \mathcal{O}_{g} \stackrel{\simeq}{\longrightarrow} \mathcal{O}_{e}, \quad\left(d L_{g}\right)_{e}: T_{e} G \stackrel{\simeq}{\longrightarrow} T_{g} G .
$$

(2) $g \mapsto \operatorname{sdim}_{g} G$ is constant; see Definition 4.3 .

The last constant value is denoted by $\operatorname{sdim} G$.

Let $G=(|G|, \mathcal{O})$ be a super Lie group. The product and the inverse on the underlying group $|G|$ give morphisms

$$
\mu: G \times G \rightarrow G, \mu(g, h)=g h ; \quad \iota: G \rightarrow G, \iota(g)=g^{-1}
$$

in SMFD. The associated sheaf-morphisms are denoted by

$$
\Delta=\mu^{*}: \mathcal{O} \rightarrow \mu_{*} \mathcal{O}_{G \times G}, \quad S=\iota^{*}: \mathcal{O} \rightarrow \iota_{*} \mathcal{O} .
$$

Recall that for every $g \in|G|, \mathcal{O}_{g}$ is a smooth Noetherian connected superalgebra with the completion $\hat{\mathcal{O}}_{g}$. We let

$$
\epsilon_{g}: \mathcal{O}_{g} \rightarrow \mathbb{k}, \quad \hat{\epsilon}_{g}: \hat{\mathcal{O}}_{g} \rightarrow \mathbb{k}
$$

denote the projections to the residue field. By (4.10) we have the identification $\hat{\mathcal{O}}_{g} \hat{\otimes} \hat{\mathcal{O}}_{h}=\hat{\mathcal{O}}_{G \times G,(g, h)}$, where $g, h \in|G|$. Using this we have the super-algebra map

$$
\hat{\Delta}_{g, h}: \hat{\mathcal{O}}_{g h} \rightarrow \hat{\mathcal{O}}_{g} \hat{\otimes} \hat{\mathcal{O}}_{h}
$$

The following is easy to see.

Lemma 5.4. $\left(\hat{\mathcal{O}}_{e}, \hat{\Delta}_{e, e}\right)$ is a smooth Noetherian complete Hopf super-algebra (see Definition 3.3), which has $\hat{\epsilon}_{e}$ and $\hat{S}_{e}$ as its counit and antipode, respectively.

Remark 5.5. (1) Let $g, h \in|G|$. It is easy to show the formulas

$$
\begin{gathered}
\left(\widehat{L_{g}^{*}}\right)_{h}=\left(\hat{\epsilon}_{g} \hat{\otimes} \operatorname{id}_{\hat{\mathcal{O}}_{h}}\right) \circ \hat{\Delta}_{g, h}, \quad\left(\widehat{R}_{g}^{*}\right)_{h}=\left(\operatorname{id}_{\hat{\mathcal{O}}_{h}} \hat{\otimes} \hat{\epsilon}_{g}\right) \circ \hat{\Delta}_{h, g}, \\
\left(\widehat{L_{g}^{*}}\right)_{h} \circ \hat{S}_{g h}=\hat{S}_{h} \circ\left(\widehat{R_{g^{-1}}^{*}}\right)_{h^{-1}} .
\end{gathered}
$$

(2) Let $G=(|G|, \mathcal{O})$, and $G^{\prime}=\left(\left|G^{\prime}\right|, \mathcal{O}^{\prime}\right)$ be super Lie groups, and let $\Delta$, $S$ and $\Delta^{\prime}, S^{\prime}$ denote the associated sheaf-morphisms as given by (5.1). A morphism $\phi: G \rightarrow G^{\prime}$ in SLG is a morphism of super manifolds such that $|\phi|$ is a group homomorphism and the sheaf-morphism $\phi^{*}: \mathcal{O}^{\prime} \rightarrow \phi_{*} \mathcal{O}$ is compatible with $\Delta^{\left({ }^{\prime}\right)}$. We claim that this $\phi^{*}$ is necessarily compatible with $S^{\left({ }^{\prime}\right)}$. Let $g^{\prime}$ denote $|\phi|(g)$ for every $g \in|G|$. To prove the claim it suffices, passing to the completed stalks, to prove

$$
\widehat{S}_{g} \circ \hat{\phi}_{g^{-1}}^{*}=\hat{\phi}_{g}^{*} \circ \widehat{S}_{g^{\prime}}^{\prime}, \quad g \in|G| .
$$

When $g=e$, this holds, since the super-algebra map $\hat{\phi}_{e}^{*}: \widehat{\mathcal{O}}_{e}^{\prime} \rightarrow \widehat{\mathcal{O}}_{e}$ between the complete Hopf super-algebras is compatible with the coproducts $\widehat{\Delta}_{e, e}^{(\prime)}$, and hence is necessarily compatible with the antipodes $\widehat{S}_{e}^{(')}$. This, together 
with the formula (5.5) applied when $h=e$, reduces the proof to proving the formulas

$$
\left(\widehat{L_{g}^{*}}\right)_{e} \circ \hat{\phi}_{g}^{*}=\hat{\phi}_{e}^{*} \circ\left(\widehat{L_{g^{\prime}}^{*}}\right)_{e}, \quad\left(\widehat{R_{g}^{*}}\right)_{e} \circ \hat{\phi}_{g}^{*}=\hat{\phi}_{e}^{*} \circ\left(\widehat{R_{g^{\prime}}^{*}}\right)_{e}
$$

for $g \in|G|$. By Remark 3.1 we have $\hat{\epsilon}_{g} \circ \hat{\phi}_{g}^{*}=\hat{\epsilon}_{g^{\prime}}$ for $g \in|G|$. Since in addition, the compatibility of $\phi^{*}$ with $\Delta^{\left({ }^{\prime}\right)}$ is equivalent to

$$
\widehat{\Delta}_{g, h} \circ \hat{\phi}_{g h}^{*}=\left(\hat{\phi}_{g}^{*} \hat{\otimes} \hat{\phi}_{h}^{*}\right) \circ \widehat{\Delta}_{g^{\prime}, h^{\prime}}^{\prime}, \quad g, h \in|G|,
$$

the desired formulas (5.6) follow from (5.4).

An alternative, shorter proof of the claim is the following. Note that $G$ represents the group-valued functor $\operatorname{Mor}(-, G)$ defined on SMFD. Since $\phi^{*}$ is assumed to be compatible with $\Delta^{\left({ }^{\prime}\right)}$, the natural transformation $\operatorname{Mor}(-, \phi)$ : $\operatorname{Mor}(-, G) \rightarrow \operatorname{Mor}\left(-, G^{\prime}\right)$ induced by $\phi$ preserves the product, or namely, for every $X \in \operatorname{SMFD}$, the map $\operatorname{Mor}(X, \phi)$ between groups preserves the product. It necessarily preserves the inverse, and sends in particular, the inverse of $\operatorname{id}_{G}$ to the inverse of $\phi$. This shows the desired compatibility.

Let $G=(|G|, \mathcal{O})$ be a super Lie group. By Lemma 5.4 and Theorem 3.15 , $\left(\hat{\mathcal{O}}_{e}\right)^{\star}$ is a smooth hyper-super-algebra of finite type, and hence $P\left(\left(\hat{\mathcal{O}}_{e}\right)^{\star}\right)$ is a finite-dimensional Lie super-algebra.

Definition 5.6. We call

$$
\operatorname{hy}(G)=\left(\hat{\mathcal{O}}_{e}\right)^{\star}, \quad \operatorname{Lie}(G)=P\left(\left(\hat{\mathcal{O}}_{e}\right)^{\star}\right)
$$

the hyper-super-algebra of $G$, and the Lie super-algebra of $G$, respectively. Note that $\operatorname{Lie}(G)$ is identified with $T_{e} G$.

The following is easy to see.

Proposition 5.7. hy and Lie define functors from SLG to the category of smooth hyper-super-algebras of finite type, and to the category of finitedimensional Lie super-algebras, respectively. Given a morphism $\phi: G \rightarrow G^{\prime}$ of super Lie groups, we have

$$
\operatorname{hy}(\phi)=\left(\phi_{e}^{*}\right)^{\star}, \quad \operatorname{Lie}(\phi)=\left.\operatorname{hy}(\phi)\right|_{\operatorname{Lie}(G)}=d \phi_{e} .
$$

Proposition 5.8. Let $G$ be a super Lie group. The hyper-algebra hy $\left(G_{\mathrm{red}}\right)$ of the Lie group $G_{\mathrm{red}}$ is the largest purely even super-subcoalgebra hy $(G)$ of hy $(G)$, while the Lie algebra $\operatorname{Lie}\left(G_{\mathrm{red}}\right)$ of $G_{\mathrm{red}}$ is the even component $\left.\overline{\operatorname{Lie}(G}\right)_{0}$ of $\operatorname{Lie}(G)$. We thus have

$$
\operatorname{hy}\left(G_{\text {red }}\right)=\underline{\operatorname{hy}(G)}, \quad \operatorname{Lie}\left(G_{\text {red }}\right)=\operatorname{Lie}(G)_{0} .
$$

Proof. This follows from Theorem 3.15 and (4.5).

Lemma 5.9. A morphism $\phi: G \rightarrow G^{\prime}$ of super Lie groups is an isomorphism if and only if the associated morphisms

$$
\phi_{\text {red }}: G_{\text {red }} \rightarrow G_{\text {red }}^{\prime}, \quad \operatorname{Lie}(\phi)_{1}: \operatorname{Lie}(G)_{1} \rightarrow \operatorname{Lie}\left(G^{\prime}\right)_{1}
$$

of Lie groups and of super-vector spaces are both isomorphisms.

Proof. To prove the non-trivial "if" part assume that $\phi_{\text {red }}$ and $\operatorname{Lie}(\phi)_{1}$ are isomorphisms. By the assumption on $\phi_{\text {red }}$ it suffices to prove that the sheafmorphism $\phi^{*}: \mathcal{O}_{G^{\prime}} \rightarrow \phi_{*} \mathcal{O}_{G}$ is locally isomorphic, in fact at $e$ by Lemma 5.3 
(1). The same argument as proving Theorem 4.5 (see the last two paragraphs of the proof) shows: $(1) \operatorname{gr}\left(\phi_{e}^{*}\right): \operatorname{gr}\left(\mathcal{O}_{G^{\prime}, e}\right) \rightarrow \operatorname{gr}\left(\mathcal{O}_{G, e}\right)$ is isomorphic since it is so in degree 0,1 (as for degree 1, this is ensured by the assumption on $\left.\operatorname{Lie}(\phi)_{1}\right)$, and (2) the result implies the desired local-isomorphy.

5.2. The Hopf algebra of representative functions. In this subsection we let $F=(|F|, \mathcal{F})$ be a Lie group.

A matrix representation $\pi: F \rightarrow \mathrm{GL}_{r}(\mathbb{k})$ of $F$ is said to be analytic, if the composites $\operatorname{pr}_{i j} \circ \pi$, where $\operatorname{pr}_{i j}: \mathrm{GL}_{r}(\mathbb{k}) \rightarrow \mathbb{k}$ projects the $(i, j)$-th entry, $1 \leq i, j \leq r$, are all analytic, or are contained in $\mathcal{F}(|F|)$. A finitedimensional left super-module $M$ over the group $F$ is said to be analytic, if the matrix representation associated with $M$ with respect to some/any basis is analytic. A left $F$-super-module is said to be analytic, if it is a union of finite-dimensional $F$-super-submodules which are analytic. An analytic right $F$-super-module is defined analogously.

Convention 5.10. By left or right F-(super-) modules we will mean analytic ones until the end of Section 6 .

Those left (resp., right) $F$-super-modules naturally form a symmetric tensor category, which we denote by

$$
\text { F-SMod (resp., SMod-F). }
$$

Recall the analogous notation (2.1), which will be used soon below.

Let $\mathcal{R}(F)$ denote the (possibly, infinitely generated) Hopf algebra of all analytic representative functions on $F$; this is by definition the $\operatorname{sum} \sum_{\pi} \operatorname{Im} \pi^{*}$ of the images $\operatorname{Im} \pi^{*}$ in $(\mathbb{k} F)^{*}$, where $\pi$ runs over all analytic matrix representations $F \rightarrow \mathrm{GL}_{r}(\mathbb{k})$ of arbitrary degree $r$, and the duals of their linearizations $\mathbb{k} F \rightarrow \mathrm{M}_{r}(\mathbb{k})$ are denoted by $\pi^{*}: \mathrm{M}_{r}(\mathbb{k})^{*} \rightarrow(\mathbb{k} F)^{*}$. This $\mathcal{R}(F)$ is a Hopf subalgebra of the dual Hopf algebra $(\mathbb{k} F)^{\circ}$ of $\mathbb{k} F$; see 21, Section 6.2 . Since $\mathbb{k} F$, being spanned by group-likes, is cocommutative, $\mathcal{R}(F)$ is commutative. We have the canonical isomorphisms

$$
F-\text { SMod } \simeq \operatorname{SComod}-\mathcal{R}(F), \quad \text { SMod- } F \simeq \mathcal{R}(F) \text {-SComod } .
$$

of symmetric tensor categories.

We have $\mathcal{R}(F) \subset \mathcal{F}(|F|)$. This, composed with the canonical map $\mathcal{F}(|F|) \rightarrow$ $\hat{\mathcal{F}}_{e}$, leads to an algebra map $\mathcal{R}(F) \rightarrow \hat{\mathcal{F}}_{e}$.

Lemma 5.11. The thus obtained $\mathcal{R}(F) \rightarrow \hat{\mathcal{F}}_{\text {e }}$ is a Hopf algebra map (see Remark 3.4 (3)), through which a (left or right) $\mathcal{R}(F)$-super-comodule turns into a discrete topological $\hat{\mathcal{F}}_{e}$-super-comodule. The construction is functorial, and results as symmetric tensor functors

$$
F \text {-SMod } \rightarrow \text { hy }(F) \text {-SMod, } \quad \operatorname{SMod}-F \rightarrow \operatorname{SMod-hy}(F) .
$$

Proof. Note that $\mathcal{R}(F) \otimes \mathcal{R}(F)$ is naturally embedded into $\mathcal{F}_{F \times F}(|F| \times|F|)$. Since the coproduct $\Delta(p)$ of $p \in \mathcal{R}(F)$, regarded as an analytic function on $|F| \times|F|$, takes the value $p(g h)$ at $(g, h) \in|F| \times|F|$, the map $\mathcal{R}(F) \rightarrow \hat{\mathcal{F}}_{e}$ preserves the coproduct. It is easily seen to preserve the counit, as well. The functors arise by Proposition [2.3, since $\hat{\mathcal{F}}_{e}=$ hy $(F)^{*}$. 
Remark 5.12. (1) One may present the construction above alternatively, as follows. The map $\mathcal{R}(F) \rightarrow \hat{\mathcal{F}}_{e}=\operatorname{hy}(F)^{*}$ naturally derives a Hopf pairing

$$
\langle,\rangle: \operatorname{hy}(F) \times \mathcal{R}(F) \rightarrow \mathbb{k} .
$$

Thus, this is a bilinear form which satisfies

$$
\langle a, p q\rangle=\left\langle a_{(1)}, p\right\rangle\left\langle a_{(2)}, q\right\rangle, \quad\langle a, 1\rangle=\epsilon(a), \quad a \in \operatorname{hy}(F), p, q \in \mathcal{R}(F)
$$

and the mirror-symmetric conditions. To an object $\mathrm{V} \in \mathrm{SMod}-F$ given by a left $\mathcal{R}(F)$-super-comodule structure

$$
\mathrm{V} \rightarrow \mathcal{R}(F) \otimes \mathrm{V}, v \mapsto v^{(-1)} \otimes v^{(0)},
$$

the second functor above associates the right hy $(F)$-super-module structure

$$
v \triangleleft a=\left\langle a, v^{(-1)}\right\rangle v^{(0)}, \quad v \in \mathrm{V}, a \in \operatorname{hy}(F) .
$$

(2) Let $\mathfrak{f}=\operatorname{Lie}(F)$. We have a canonical hyper-algebra map $\mathfrak{U}(\mathfrak{f}) \rightarrow$ hy $(F)$; see (3.4). The associated, restriction functors extend the symmetric tensor functors (5.10) so as (for the second)

$$
\text { SMod- } F \rightarrow \operatorname{SMod}-\operatorname{hy}(F) \rightarrow \operatorname{SMod}-\mathfrak{U}(\mathfrak{f}) \text {. }
$$

5.3. The hy $(G)$-action on $\mathcal{O}$. Let $G=(|G|, \mathcal{O})$ be a super Lie group. Let $g \in|G|$. By $\hat{\Delta}_{g, e}: \hat{\mathcal{O}}_{g} \rightarrow \hat{\mathcal{O}}_{g} \hat{\otimes} \hat{\mathcal{O}}_{e}, \hat{\mathcal{O}}_{g}$ is a topological right $\hat{\mathcal{O}}_{e^{- \text {super- }}}$ comodule; it is in fact an algebra-object in the tensor category of those super-comodules. By Proposition 3.18 for $A$ applied to $\hat{\mathcal{O}}_{e}$, it follows that $\hat{\mathcal{O}}_{g}$ turns into a left hy $(G)$-super-module super-algebra. Here one should recall hy $(G)=\left(\hat{\mathcal{O}}_{e}\right)^{\star}$, and note that every element $a \in$ hy $(G)$ gives a continuous linear map $\hat{\mathcal{O}}_{e} \rightarrow \mathbb{k}$. The result is formulated as follows.

Lemma 5.13. Let $g \in|G|$. Let every element a of hy $(G)$ act on $\hat{\mathcal{O}}_{g}$ as the continuous super-linear endomorphism

$$
\hat{\mathcal{O}}_{g} \stackrel{\hat{\Delta}_{g, e}}{\longrightarrow} \hat{\mathcal{O}}_{g} \hat{\otimes} \hat{\mathcal{O}}_{e} \stackrel{\operatorname{id} \hat{\otimes} a}{\longrightarrow} \hat{\mathcal{O}}_{g} \hat{\otimes} \mathbb{k}=\hat{\mathcal{O}}_{g} .
$$

Then $\hat{\mathcal{O}}_{g}$ turns into a left hy $(G)$-super-module super-algebra.

Proposition 5.14. Let $U \subset|G|$ be an open set. Through the natural embed$\operatorname{ding} \mathcal{O}(U) \rightarrow \prod_{g \in U} \hat{\mathcal{O}}_{g}, \mathcal{O}(U)$ is a super-submodule of the product $\prod_{g \in U} \hat{\mathcal{O}}_{g}$ of the left hy $(G)$-super-modules $\hat{\mathcal{O}}_{g}, g \in U$. Therefore, $\mathcal{O}(U)$ turns into a left hy $(G)$-super-module super-algebra.

Proof. Suppose $m \mid n=\operatorname{dim} G$. Let $G_{\text {red }}=(|G|, \mathcal{F})$, where $\mathcal{F}=\mathcal{O}_{\text {red }}$, denote the Lie group associated with $G$. We may suppose $\mathcal{O}_{e}=\mathcal{F}_{e} \otimes \wedge(\mathrm{W})$, where $\mathrm{W}$ is a vector space of dimension $n$, and hence hy $(G)=\mathcal{F}_{e}^{\star} \otimes \wedge(\mathrm{W})^{*}$. Let $\mathfrak{n}_{e}$ be the maximal ideal of $\mathcal{F}_{e}$.

Let $a \in \operatorname{hy}(G)$. Then there exists a positive integer $\ell$ such that $a\left(\hat{\mathfrak{n}}_{e}^{\ell} \otimes\right.$ $\wedge(\mathrm{W}))=0$, or in other words, $a \in\left(\mathcal{F}_{e} / \mathfrak{n}_{e}^{\ell} \otimes \wedge(\mathrm{W})\right)^{*}$. Set

$$
\mathrm{M}_{e}:=\mathcal{F}_{e} / \mathfrak{n}_{e}^{\ell} \otimes \wedge(\mathrm{W}) .
$$

This $\mathrm{M}_{e}$ is a finite-dimensional super-vector space. To show that the action by a stabilizes $\mathcal{O}(U)$ in $\prod_{g \in U} \hat{\mathcal{O}}_{g}$, it suffices to prove that the composite

$$
\mathcal{O}(U) \rightarrow \prod_{g \in U} \hat{\mathcal{O}}_{g} \stackrel{\hat{\Delta}_{g, e}}{\longrightarrow} \prod_{g \in U}\left(\hat{\mathcal{O}}_{g} \hat{\otimes} \hat{\mathcal{O}}_{e}\right) \rightarrow \prod_{g \in U}\left(\hat{\mathcal{O}}_{g} \otimes \mathrm{M}_{e}\right)=\left(\prod_{g \in U} \hat{\mathcal{O}}_{g}\right) \otimes \mathrm{M}_{e}
$$


where the last arrow arises from the natural projection $\hat{\mathcal{O}}_{e}=\hat{\mathcal{F}}_{e} \otimes \wedge(\mathrm{W}) \rightarrow$ $\mathcal{F}_{e} / \mathfrak{n}_{e}^{\ell} \otimes \wedge(\mathrm{W})=\mathrm{M}_{e}$ (see (2.4) $)$, takes values in $\mathcal{O}(U) \otimes \mathrm{M}_{e}$.

Let $s=\operatorname{dim} \mathrm{M}_{e}$. Given a basis $m_{1}, \ldots, m_{s}$ of $\mathrm{M}_{e}$, let $p \in \mathcal{O}(U)$, and denote its image in $\prod_{g \in U} \hat{\mathcal{O}}_{g} \otimes \mathrm{M}_{e}$ by $\sum_{i=1}^{s}\left(p_{g, i}\right)_{g \in U} \otimes m_{i}$ with $p_{g, i} \in \hat{\mathcal{O}}_{g}$. We wish to show that every $g_{0} \in U$ has an open neighborhood $V(\subset U)$ such that for every $i$, the elements $p_{g, i}, g \in V$, arise (necessarily uniquely) from some $p_{V, i}$ in $\mathcal{O}(V)$.

Let $g_{0} \in U$. There exist neighborhoods $V$ of $g_{0}$ and $V^{\prime}$ of $e$, such that $V \subset U$ and $V V^{\prime} \subset U$. The desired result will follow if we prove that the map

$$
\mathcal{O}(U) \rightarrow \prod_{g \in V} \hat{\mathcal{O}}_{g} \otimes \mathrm{M}_{e}
$$

analogous to the composite above takes values in $\mathcal{O}(V) \otimes \mathrm{M}_{e}$. Since the map naturally factors through

$$
\mathcal{O}_{G \times G}\left(V \times V^{\prime}\right) \rightarrow \prod_{g \in V} \hat{\mathcal{O}}_{G \times G,(g, e)}=\prod_{g \in V} \hat{\mathcal{O}}_{g} \hat{\otimes} \hat{\mathcal{O}}_{e} \rightarrow \prod_{g \in V} \hat{\mathcal{O}}_{g} \otimes \mathrm{M}_{e}
$$

it suffices to prove that this last composite takes values in $\mathcal{O}(V) \otimes \mathrm{M}_{e}$. We may re-choose $V$ and $V^{\prime}$ so that they are open sub-manifolds of $\mathbb{k}^{m}$, and $\left(V,\left.\mathcal{O}\right|_{V}\right)$ and $\left(V^{\prime},\left.\mathcal{O}\right|_{V^{\prime}}\right)$ are s-split, whence in particular,

$$
\mathcal{O}(V)=\mathcal{F}(V) \otimes \wedge(\mathrm{W})
$$

where $\mathrm{W}$ is as before.

We use the operators $\Delta^{k}$ on $\mathcal{F}\left(V \times V^{\prime}\right)\left(=\mathcal{F}_{\mathbb{R}^{2 m}}\left(V \times V^{\prime}\right)\right)$ used in the proof of Proposition 4.6. As $\boldsymbol{k}$ we use only the multi-indexes of the form $(\mathbf{0}, \boldsymbol{i})$, where $\mathbf{0}=(0, \ldots, 0)$ and $\boldsymbol{i}=\left(i_{1}, \ldots, i_{m}\right)$ both have $m$ entries. Suppose $\mathcal{F}_{e}=\mathbb{k}\left\{\mathrm{T}_{1}, \ldots, \mathrm{T}_{m}\right\}$ naturally. Then $\mathcal{F}_{e} / \mathfrak{n}_{e}^{\ell}$ has $\mathrm{T}^{i} \bmod \mathfrak{n}_{e}^{\ell},|\boldsymbol{i}|<\ell$, as a basis, where $T^{i}=T_{1}^{i_{1}} \ldots T_{m}^{i_{m}}$. Let

$$
\sigma: \mathcal{F}\left(V \times V^{\prime}\right) \rightarrow \mathcal{F}(V) \otimes \mathcal{F}_{e} / \mathfrak{n}_{e}^{\ell}
$$

be the map which associates to each $f=f(g, h) \in \mathcal{F}\left(V \times V^{\prime}\right)$, the sum

$$
\left.\sum_{|i|<\ell} \Delta^{(\mathbf{0}, i)} f\right|_{h=e} \otimes T^{i} \bmod \mathfrak{n}_{e}^{\ell}
$$

As is seen from (4.9), if $\left(\left.\Delta^{(\mathbf{0}, i)} f\right|_{h=e}\right)_{g}\left(\in \mathcal{F}_{g}\right)$ denotes the natural image of $\left.\Delta^{(\mathbf{0}, i)} f\right|_{h=e}(\in \mathcal{F}(V))$, then the natural image $f_{(g, e)}\left(\in \mathcal{F}_{(g, e)}\right)$ of $f$ equals the sum $\sum_{i}\left(\left.\Delta^{(\mathbf{0}, i)} f\right|_{h=e}\right)_{g} \top^{i}$. We have proved that the natural map $\mathcal{F}\left(V \times V^{\prime}\right) \rightarrow$ $\prod_{g \in V} \hat{\mathcal{F}}_{g} \otimes \mathcal{F}_{e} / \mathfrak{n}_{e}^{\ell}$ factors through $\sigma$, whence it takes values in $\mathcal{F}(V) \otimes \mathcal{F}_{e} / \mathfrak{n}_{e}^{\ell}$.

The composite (5.15) is identified with

$$
\begin{aligned}
\mathcal{F}\left(V \times V^{\prime}\right) \otimes \wedge(\mathrm{W}) \otimes \wedge(\mathrm{W}) \stackrel{\sigma \otimes \mathrm{id}}{\longrightarrow} & \mathcal{F}(V) \otimes \mathcal{F}_{e} / \mathfrak{n}_{e}^{\ell} \otimes \wedge(\mathrm{W}) \otimes \wedge(\mathrm{W}) \\
& =\mathcal{O}(V) \otimes \mathrm{M}_{e} \rightarrow \prod_{g \in V} \hat{\mathcal{O}}_{g} \otimes \mathrm{M}_{e},
\end{aligned}
$$

where the last equality is given by (5.16) and (5.14). Therefore, the map (5.15) takes values in $\mathcal{O}(V) \otimes \mathcal{F}_{e} / \mathfrak{n}_{e}^{\ell}$, as desired.

Corollary 5.15. With each $\mathcal{O}(U)$ given the structure of a left hy $(G)$-supermodule super-algebra as above, $\mathcal{O}$ is a sheaf of such super-algebras. 
Proof. Indeed, the given structure is compatible with the restriction maps $\mathcal{O}(U) \rightarrow \mathcal{O}(V)$ associated with an inclusion $U \supset V$ of open sets.

Example 5.16. Let $m$ be a positive integer. Let $F=\left(\mathbb{k}^{m}, \mathcal{F}\right)$ be the Lie group constructed on $\mathbb{k}^{m}$ with respect to the addition. Note that $\hat{\mathcal{F}}_{e}=$ $\mathbb{k}\left[\left[\mathrm{P}_{1}, \ldots, \mathrm{P}_{m}\right]\right]$ with $\mathrm{P}_{i}$ primitive, and the hyper-algebra hy $(F)$ of $F$ is the commutative hyper-algebra with basis

$$
b_{\boldsymbol{k}}=b_{k_{1}}^{(1)} \ldots b_{k_{m}}^{(m)}, \quad \boldsymbol{k}=\left(k_{1}, \ldots, k_{m}\right), k_{i} \geq 0
$$

such that

$$
b_{\boldsymbol{k}}\left(\mathrm{P}^{j}\right)=\delta_{\boldsymbol{k}, \boldsymbol{j}}, \text { where } \mathrm{P}^{j}=\mathrm{P}_{1}^{j_{1}} \ldots \mathrm{P}_{m}^{j_{m}},
$$

and each sequence $b_{0}^{(i)}, b_{1}^{(i)}, b_{2}^{(i)}, \ldots, 1 \leq i \leq m$, satisfies (3.6). By Corollary 5.15 applied in the purely even situation, $\mathcal{F}$ is a sheaf of left hy $(F)$-module algebras. The action $b_{\boldsymbol{k}}: \mathcal{F} \rightarrow \mathcal{F}$ by the basis element coincides with the operator $\Delta^{k}$, as is seen from the formula

$$
\hat{\Delta}_{g, e}\left(\mathrm{~T}^{i}\right)=\sum_{i \geq j}\left(\begin{array}{l}
\boldsymbol{i} \\
\boldsymbol{j}
\end{array}\right) \mathrm{T}^{i-j} \otimes \mathrm{P}^{j}, \quad g \in \mathbb{k}^{m}
$$

compared with (4.9), where we suppose $\hat{\mathcal{F}}_{g}=\mathbb{k}\left[\left[\mathrm{T}_{1}, \ldots, \mathrm{T}_{m}\right]\right]$ naturally.

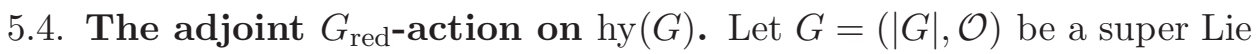
group, and let $G_{\text {red }}=(|G|, \mathcal{F})$, where $\mathcal{F}=\mathcal{O}_{\text {red }}$, denote the associated Lie group. Suppose $g \in|G|$. Recall from (4.4) that $\left(\hat{\mathcal{O}}_{g}\right)_{\text {red }}=\hat{\mathcal{F}}_{g}$, and let

$$
\widehat{\operatorname{red}}_{g}: \hat{\mathcal{O}}_{g} \rightarrow \hat{\mathcal{F}}_{g}
$$

denote the natural projection.

The right $G$-adjoint action $G \times G \rightarrow G,(h, g) \mapsto g^{-1} h g$ on $G$ is a morphism in SMFD, which induces a super-algebra map $\hat{\mathcal{O}}_{e} \rightarrow \hat{\mathcal{O}}_{e} \hat{\otimes} \hat{\mathcal{O}}_{g}$ for each $g \in|G|$. The composite of the last map with id $\hat{\otimes} \widehat{\operatorname{red}}_{g}$ is denoted by

$$
\alpha_{g}: \hat{\mathcal{O}}_{e} \rightarrow \hat{\mathcal{O}}_{e} \hat{\otimes} \hat{\mathcal{F}}_{g},
$$

whose composite with id $\otimes \hat{\epsilon}_{g}: \hat{\mathcal{O}}_{e} \hat{\otimes} \hat{\mathcal{F}}_{g} \rightarrow \hat{\mathcal{O}}_{e} \hat{\otimes} \mathbb{k}=\hat{\mathcal{O}}_{e}$ is denoted by

$$
\beta_{g}: \hat{\mathcal{O}}_{e} \rightarrow \hat{\mathcal{O}}_{e} \text {. }
$$

Since one sees that $\alpha_{g}$ is compatible with $\hat{\Delta}_{e, e}$ on $\hat{\mathcal{O}}_{e}$, it follows that $\beta_{g}$ is an endomorphism of the Noetherian complete Hopf algebra $\hat{\mathcal{O}}_{e}$. Let

$$
\gamma_{g}=\left(\beta_{g}\right)^{\star}: \operatorname{hy}(G) \rightarrow \operatorname{hy}(G)
$$

denote the corresponding endomorphism of the hyper-super-algebra hy $(G)$.

Proposition 5.17. We have the following.

(1) By $\beta_{g}, g \in|G|$, the abstract group $G$ acts on $\hat{\mathcal{O}}_{e}$ from the left as automorphisms of the complete Hopf algebra.

(2) By $\gamma_{g}, g \in|G|$, the Lie group $G_{\text {red }}$ acts analytically on $\operatorname{hy}(G)$ from the right as automorphisms of the hyper-super-algebra. Thus, hy $(G)$ turns into a Hopf-algebra object in SMod-Gred. 
(3) By Lemma5.11 there is induced a right hy $\left(G_{\mathrm{red}}\right)$-super-module structure on hy $(G)$, by which hy $(G)$ turns, moreover, into a Hopf-algebra object in $\mathrm{SMod}-\mathrm{hy}\left(G_{\mathrm{red}}\right)$. The induced action is precisely the right adjoint action $\operatorname{ad}_{r}(a), a \in$ hy $\left(G_{\text {red }}\right)$, given by

$$
\operatorname{ad}_{r}(a)(b)=S\left(a_{(1)}\right) b a_{(2)}, \quad b \in \operatorname{hy}(G) .
$$

Proof. (1) We see

$$
\beta_{g}=\left(\widehat{L_{g^{-1}}^{*}}\right)_{e} \circ\left(\widehat{R_{g}^{*}}\right)_{e}=\left(\widehat{R_{g}^{*}}\right)_{e} \circ\left(\widehat{L_{g^{-1}}^{*}}\right)_{e},
$$

which implies that $\beta_{e}=$ id and $\beta_{g h}=\beta_{g} \circ \beta_{h}, g, h \in|G|$. This proves Part 1.

(2) The last equalities shows that $\gamma_{e}=\mathrm{id}$ and $\gamma_{g h}=\gamma_{h} \circ \gamma_{g}, g, h \in|G|$.

It remains to prove that the $G_{\mathrm{red}}$-action on hy $(G)$ is analytic. Fix $\ell>0$. Then maps $\alpha_{g}, g \in|G|$, induce super-algebra maps

$$
\alpha_{g}^{\prime}: \mathcal{O}_{e} / \mathfrak{m}_{e}^{\ell} \rightarrow \mathcal{O}_{e} / \mathfrak{m}_{e}^{\ell} \otimes \hat{\mathcal{F}}_{g}, \quad g \in|G| .
$$

Let $s=\operatorname{dim} \mathcal{O}_{e} / \mathfrak{m}_{e}^{\ell}$. Choose a basis $z_{1}, \ldots, z_{s}$ of $\mathcal{O}_{e} / \mathfrak{m}_{e}^{\ell}$, and write $\alpha_{g}^{\prime}\left(z_{j}\right)=$ $\sum_{i=1}^{s} z_{i} \otimes p_{g, i j}$ with $p_{g, i j} \in \hat{\mathcal{F}}_{g}$. We have an open neighborhood $U$ of $e$ such that $z_{j}, 1 \leq j \leq s$, are all contained in the image of the natural composite $\mathcal{O}(U) \rightarrow \hat{\mathcal{O}}_{e} \rightarrow \mathcal{O}_{e} / \mathfrak{m}_{e}^{\ell}$. Let $g_{0} \in|G|$. Then there exist neighborhoods $U^{\prime}$ of $e$ and $V$ of $g_{0}$, such that $U^{\prime} \times V$ is mapped to $U$ by the $G$-adjoint action, $\left(U^{\prime},\left.\mathcal{O}\right|_{U^{\prime}}\right)$ is s-split, and $U_{\text {red }}^{\prime}$ and $V$ are open sub-manifolds of $\mathbb{k}^{m}$, $m=\operatorname{dim} G_{\text {red }}$. We see as in the proof of Proposition 5.14 that the natural $\operatorname{map} \mathcal{O}_{G \times G_{\text {red }}}\left(U^{\prime} \times V\right) \rightarrow \mathcal{O}_{e} / \mathfrak{m}_{e}^{\ell} \otimes \prod_{g \in V} \hat{\mathcal{F}}_{g}$ takes values in $\mathcal{O}_{e} / \mathfrak{m}_{e}^{\ell} \otimes \mathcal{F}(V)$. It follows that the composite

$$
\mathcal{O}(U) \rightarrow \hat{\mathcal{O}}_{e} \rightarrow \mathcal{O}_{e} / \mathfrak{m}_{e}^{\ell} \stackrel{\left(\alpha_{g}^{\prime}\right)_{g \in V}}{\longrightarrow} \prod_{g \in V} \mathcal{O}_{e} / \mathfrak{m}_{e}^{\ell} \otimes \hat{\mathcal{F}}_{g}=\mathcal{O}_{e} / \mathfrak{m}_{e}^{\ell} \otimes \prod_{g \in V} \hat{\mathcal{F}}_{g}
$$

takes values in $\mathcal{O}_{e} / \mathfrak{m}_{e}^{\ell} \otimes \mathcal{F}(V)$. Fix $1 \leq i, j \leq s$. It follows that $p_{g, i j}, g \in V$, uniquely arise from some $p_{V, i j} \in \mathcal{F}(V)$. Note that $V$ can run over an open covering of $|G|$. All $p_{V, i j}$ are then glued together into an analytic function, say $p_{i j}$, on the whole $|G|$. Since

$$
\beta_{g}\left(z_{j}\right)=\sum_{i=1}^{s} \hat{\epsilon}_{g}\left(p_{g, i j}\right) z_{i}=\sum_{i=1}^{s} p_{i j}(g) z_{i},
$$

the $G_{\text {red-action on }} \mathcal{O}_{e} / \mathfrak{m}_{e}^{\ell}$ is given by the matrix representation $g \mapsto\left(p_{i j}(g)\right)$,

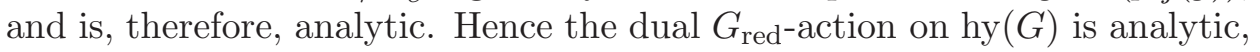
as desired.

(3) Let $F=G_{\text {red }}$. We aim to prove that the induced hy $(F)$-action hy $(G) \otimes$ hy $(F) \rightarrow$ hy $(G)$ coincides with $\left(\alpha_{e}\right)^{\star}$, which implies Part 3. With the notation above, restrict the left $\mathcal{R}(F)$-coaction on $\operatorname{hy}(G)$ to $\left(\mathcal{O}_{e} / \mathfrak{m}_{e}^{\ell}\right)^{*}$. One then sees that the dual right $\mathcal{R}(F)$-coaction $\mathcal{O}_{e} / \mathfrak{m}_{e}^{\ell} \rightarrow \mathcal{O}_{e} / \mathfrak{m}_{e}^{\ell} \otimes \mathcal{R}(F)$ induces, through the natural map $\mathcal{R}(F) \rightarrow \hat{\mathcal{F}}_{e}$, the $\alpha_{e}^{\prime}$ above. Therefore, the induced hy $(F)$-action restricted to $\left(\mathcal{O}_{e} / \mathfrak{m}_{e}^{\ell}\right)^{*}$ coincides with $\left(\alpha_{e}^{\prime}\right)^{*}$. This implies the desired result.

Remark 5.18. We call the $G_{\mathrm{red}}$-action on hy $(G)$ above, the right adjoint action. Note that by restriction, $\operatorname{Lie}(G)=P($ hy $(G))$ turns into a Lie-algebra object in SMod- $G_{\text {red }}$. The restricted action as well is called the right adjoint 
action. It follow by Proposition 5.17 (3) that the induced Lie $\left(G_{\text {red }}\right)$-action on $\operatorname{Lie}(G)$ coincides with the right adjoint action for Lie super-algebras

$$
\operatorname{ad}_{r}(x)(y)=[y, x], \quad x \in \operatorname{Lie}\left(G_{\text {red }}\right), y \in \operatorname{Lie}(G) .
$$

\section{Category equivalence SLG $\approx \mathrm{HCP}$}

6.1. Functor to Harish-Chandra pairs. The concept of Harish-Chandra pairs goes back to Kostant [7. The following version of definition is a reformulation of [12, Definition 6.4] into our analytic situation.

Let $(F, \mathrm{~V})$ be a pair of a Lie group $F$ and a finite-dimensional right $F$ module $\mathrm{V}$; see Convention 5.10. The action by $F$ on $\mathrm{V}$ (or any action by a Lie group on a super-module) will be presented as $v^{g}$, where $g \in F, v \in \mathrm{V}$. There is induced a right hy $(F)$-module structure on $\mathrm{V}$; this action as well as the restricted one by $\operatorname{Lie}(F)=P($ hy $(F)$ ) (see (P2) below) will be indicated by $\triangleleft$, as in (5.13).

Definition 6.1. (1) The pair $(F, \mathrm{~V})$ is called a Harish-Chandra pair, if it is equipped with an $F$-equivariant linear map [, ] : $\mathrm{V} \otimes \mathrm{V} \rightarrow \operatorname{Lie}(F)$, where $\operatorname{Lie}(F)$ is supposed to be given the right adjoint $F$-action, such that

$(\mathrm{P} 1)[v, w]=[w, v], \quad v, w \in \mathrm{V}$,

(P2) $v \triangleleft[v, v]=0, \quad v \in \mathrm{V}$.

(2) A morphism $(\phi, \psi):\left(F_{1}, \bigvee_{1}\right) \rightarrow\left(F_{2}, \vee_{2}\right)$ of Harish-Chandra pairs consists of a morphism $\phi: F_{1} \rightarrow F_{2}$ of Lie groups and a linear map $\psi: \bigvee_{1} \rightarrow$ $\mathrm{V}_{2}$ such that

(P3) $\psi\left(v^{g}\right)=\psi(v)^{\phi(g)}, \quad v \in \mathrm{V}_{1}, g \in F_{1}$,

(P4) $[\psi(v), \psi(w)]=\operatorname{Lie}(\phi)([v, w]), \quad v, w \in \mathrm{V}_{1}$.

Here, Lie $(\phi)$ in $(\mathrm{P} 4)$ may be replaced by hy $(\phi)$; see Remark $5.12(2)$. The composite of morphisms is defined in the obvious manner.

(3) HCP denotes the category of Harish-Chandra pairs.

Recall that SLG denotes the category of super Lie groups. Suppose $G=$ $(|G|, \mathcal{O}) \in \mathrm{SLG}$, and let $\mathfrak{g}=\operatorname{Lie}(G)$ denote the Lie super-algebra of $G$. Recall from Remark 5.18 that $\mathfrak{g}$ is a Lie-algebra object in $\mathrm{SMod}-G_{\text {red }}$ with respect to the right adjoint action by the associated Lie group $G_{\text {red }}$. Regard the odd

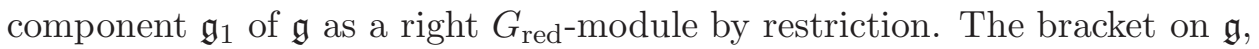
restricted to $\mathfrak{g}_{1}$, is a $G_{\text {red }}$-equivariant linear map which takes values in $\mathfrak{g}_{0}$, the Lie algebra of $G_{\text {red }}$; see (5.7).

Proposition 6.2. The pair $\left(G_{\mathrm{red}}, \mathfrak{g}_{1}\right)$, equipped with the restricted bracket, turns into a Harish-Chandra pair. Moreover, this construction gives rise to the functor

$$
\Phi: \mathrm{SLG} \rightarrow \mathrm{HCP}, \Phi(G)=\left(G_{\mathrm{red}}, \operatorname{Lie}(G)_{1}\right) .
$$

Proof. Obviously, $(\mathrm{P} 1)$ is satisfied. By Remark 5.18 , (P2) now reads $[v,[v, v]]=$ 0 , which holds in $\operatorname{Lie}(G)$. Since the assignment $G \mapsto\left(\operatorname{Lie}(G)\right.$ in $\left.\operatorname{SMod}-G_{\text {red }}\right)$ is functorial, so is $\Phi$ as well.

Theorem 6.3. The functor $\Phi: \mathrm{SLG} \rightarrow \mathrm{HCP}$ is an equivalence.

Modifying the construction in [11] we will construct an explicit quasiinverse functor. 
6.2. Construction of a quasi-inverse. Let $(F, \mathrm{~V}) \in \mathrm{HCP}$. Let $\mathcal{F}$ and $D=\operatorname{hy}(F)$ denote the structure sheaf of $F$ and the hyper-algebra of $F$, respectively.

By [11, Theorem 10] we have a unique hyper-super-algebra $C$ with the properties: (1) $\underline{C}=D, P(C)_{1}=\mathrm{V}$, and (2) $C$ is generated by $D$ and $\mathrm{V}$ (to be more precise, $C$ is generated by $\vee$ as a $D$-ring) and is defined by the relations

$$
v a=a_{(1)}\left(v \triangleleft a_{(2)}\right), \quad v w+w v=[v, w],
$$

where $v, w \in \mathrm{V}$ and $a \in D$. Choosing arbitrarily a basis $\left(v_{i}\right)_{1 \leq i \leq n}, n=$ $\operatorname{dim} \mathrm{V}$, of $\mathrm{V}$, we have as in (3.10), the unit-preserving isomorphism of left $D$-super-module super-coalgebras

$$
D \otimes \wedge(\mathrm{V}) \stackrel{\simeq}{\longrightarrow} C, \quad a \otimes\left(v_{i_{1}} \wedge v_{i_{2}} \wedge \cdots \wedge v_{i_{r}}\right) \mapsto a v_{i_{1}} v_{i_{2}} \ldots v_{i_{r}}
$$

where $1 \leq i_{1}<i_{2}<\cdots<i_{r} \leq n, 0 \leq r \leq n$. Fixing an isomorphism as above we will identify so as

$$
C=D \otimes \wedge(\mathrm{V})
$$

By Proposition 5.17 (2) applied in the purely even situation, $F$ acts analytically on $D$ from the right as Hopf-algebra automorphisms.

Lemma 6.4. This $F$-action on $D$, together with the original one on $\mathrm{V}$, uniquely extend onto $C$ so that $C$ turns into a Hopf-algebra object in SMod-F.

The extended action as well will be presented as $a^{g}$, where $a \in C, g \in F$.

Recall from (5.9) that $\mathrm{V}$ is a left comodule over the Hopf algebra $\mathcal{R}(F)$ of analytic representative functions on $F$, whose structure we present as $v \mapsto v^{(-1)} \otimes v^{(0)} ;$ see (5.12).

Proof. It suffices to show that the relations given in (6.1) are compatible with the $F$-action. For the second relation this follows from the $F$-equivariance of [ , ]. We wish to prove $(v \triangleleft a)^{g}=v^{g} \triangleleft a^{g}$ for $g \in F, a \in D$ and $v \in \mathrm{V}$; this implies the compatibility for the first relation. Given $p \in \mathcal{R}(F)$, Lemma 5.11 (see also (6.16) below) shows

$$
\begin{aligned}
& \left\langle a^{g}, p\right\rangle=p_{(1)}\left(g^{-1}\right)\left\langle a, p_{(2)}\right\rangle p_{(3)}(g) \\
& \text { or } p_{(1)}(g)\left\langle a^{g}, p_{(2)}\right\rangle=\left\langle a, p_{(1)}\right\rangle p_{(2)}(g),
\end{aligned}
$$

where $\langle$,$\rangle denotes the pairing (5.11). If we write v^{(-2)} \otimes v^{(-1)} \otimes v^{(0)}$ for $\Delta\left(v^{(-1)}\right) \otimes v^{(0)}$, then the desired equality follows from the last equation and (5.13), so as

$$
\begin{aligned}
v^{g} \triangleleft a^{g} & =v^{(-2)}(g)\left\langle a^{g}, v^{(-1)}\right\rangle v^{(0)}=\left\langle a, v^{(-2)}\right\rangle v^{(-1)}(g) v^{(0)} \\
& =\left\langle a, v^{(-1)}\right\rangle\left(v^{(0)}\right)^{g}=(v \triangleleft a)^{g} .
\end{aligned}
$$

By Corollary [5.15, $\mathcal{F}$ is a sheaf of left $D$-module algebras. Given an open set $U \subset|F|$, let $\mathcal{P}(U)=\operatorname{Hom}_{D}(C, \mathcal{F}(U))$ denote the super-vector space of all left $D$-module maps $C \rightarrow \mathcal{F}(U)$, which is given the parity $\mathcal{P}(U)_{i}=$ $\operatorname{Hom}_{D}\left(C_{i}, \mathcal{F}(U)\right), i=0,1$. This forms a super-algebra with respect to the convolution-product [17, p.6], and gives rise to a presheaf

$$
\mathcal{P}=\operatorname{Hom}_{D}(C, \mathcal{F})
$$


of super-algebras on $|F|$. By (6.2) this $\mathcal{P}$ is identified with $\mathcal{F} \otimes \wedge\left(\mathrm{V}^{*}\right.$ ) (see (4.2)), so that $\mathcal{P}$ is indeed a sheaf, and $(|F|, \mathcal{P})$ is an s-split super-manifold. Given $g \in|F|, \mathcal{F}_{g}$ and $\hat{\mathcal{F}}_{g}$ are naturally (topological) left $D$-module algebra (see Lemma 5.13), and we have

$$
\mathcal{P}_{g}=\operatorname{Hom}_{D}\left(C, \mathcal{F}_{g}\right), \quad \hat{\mathcal{P}}_{g}=\operatorname{Hom}_{D}\left(C, \hat{\mathcal{F}}_{g}\right) .
$$

Let $n>0$ be an integer, and let $\mathcal{F}^{(n)}=\mathcal{F}_{F^{n}}$ denote the structure sheaf of the product $F^{n}$ of $n$ copies of the Lie group $F=(|F|, \mathcal{F})$. It is a sheaf of left $D^{\otimes n}$-module algebra, since hy $\left(F^{n}\right)=D^{\otimes n}$. The construction of $\mathcal{P}$ is generalized so that the super-algebra associated with every open set $U \subset|F|^{n}$

$$
\mathcal{P}^{(n)}(U)=\operatorname{Hom}_{D \otimes n}\left(C^{\otimes n}, \mathcal{F}^{(n)}(U)\right)
$$

which consists of all left $D^{\otimes n}$-module maps gives rise to a sheaf $\mathcal{P}^{(n)}$; by (6.2), this is identified with $\mathcal{F}^{(n)} \otimes \wedge\left(\mathrm{V}^{*}\right)^{\otimes n}\left(=\mathcal{F}^{(n)} \otimes \wedge\left(\left(\mathrm{V}^{*}\right)^{\oplus n}\right)\right)$, and is, therefore, regarded as the structure sheaf of the product $(|F|, \mathcal{P})^{n}$ of $n$ copies of $(|F|, \mathcal{P})$; see Remark $4.7(2)$.

Let $\mu: F \times F \rightarrow F, \mu(g, h)=g h$ and $\iota: F \rightarrow F, \iota(g)=g^{-1}$ denote the product and the inverse of the underlying group of $F$. The first step of making $(|F|, \mathcal{P})$ into a super Lie group is to give to it the group structure the same as $\mu, \iota$. For any open set $V \subset|F|$ we let $\langle\rangle:, V \times \mathcal{F}(V) \rightarrow \mathbb{k}$, $\langle g, p\rangle=p(g)$ denote the evaluation map. The next step is to define sheafmorphisms

$$
\Delta: \mathcal{P} \rightarrow \mu_{*} \mathcal{P}^{(2)}, \quad S: \mathcal{P} \rightarrow \iota_{*} \mathcal{P}
$$

which shall satisfy $\Delta=\mu^{*}, S=\iota^{*}$. Given open sets $U \subset|F| \times|F|, V \subset|F|$ with $\mu(U) \subset V$, and an element $p \in \mathcal{P}(V)$, we define the element $\Delta(p)$ of $\mathcal{P}^{(2)}(U)=\operatorname{Hom}_{D \otimes D}\left(C \otimes C, \mathcal{F}^{(2)}(U)\right)$ to be the map which sends $a \otimes b \in C \otimes C$ to the analytic function on $U$ with the value

$$
\Delta(p)(a \otimes b)(g, h):=\left\langle g h, p\left(a^{h} b\right)\right\rangle \text { at }(g, h) \in U .
$$

Given open sets $U, V \subset|F|$ with $\iota(U) \subset V$, and an element $p \in \mathcal{P}(V)$, we define the element $S(p)$ of $\mathcal{P}(U)=\operatorname{Hom}_{D}(C, \mathcal{F}(U))$ to be the map which sends $a \in C$ to the analytic function on $U$ with the value

$$
S(p)(a)(g):=\left\langle g^{-1}, p\left(S_{C}(a)^{g^{-1}}\right)\right\rangle \text { at } g \in U .
$$

Proposition 6.5. These indeed define sheaf-morphisms $\Delta, S$ of superalgebras, with which $(|F|, \mathcal{P})$, given the group structure $\mu, \iota$, is a super Lie group such that $\Delta=\mu^{*}, S=\iota^{*}$.

In the algebraic situation treated by [11, 13], every affine super-group scheme (see Section 2.2) is constructed uniquely from an (algebraic) HarishChandra pair. The construction is conceptual, involving quite similar expressions to (6.6) and (6.7). To prove Proposition 6.5 we choose to deduce it from the algebraic counterpart just cited, because the resulting construction is conceptual, again, and because a Hopf super-algebra, $\mathcal{B}$, which we will construct in the course, will play a role in Section 6.5.

We apply the algebraic construction of [13, Section 4.4] to the Hopf algebra $\mathcal{R}(F)$ and the hyper-super-algebra $C$ as above. The Hopf pairing 
(5.11) makes $\mathcal{R}(F)$ into a left module algebra over $D=$ hy $(F)$, so that $a \triangleright p=p_{(1)}\left\langle a, p_{(2)}\right\rangle$, where $a \in D, p \in \mathcal{R}(F)$. Let

$$
\mathcal{B}=\operatorname{Hom}_{D}(C, \mathcal{R}(F))
$$

denote the super-algebra (with respect to the convolution-product and the parity induced from $C$, as before) consisting of all left $D$-module maps $D \rightarrow$ $\mathcal{R}(F)$. Note that

$$
\mathcal{B}^{\otimes n}=\operatorname{Hom}_{D^{\otimes n}}\left(C^{\otimes n}, \mathcal{R}(F)^{\otimes n}\right), \quad n \geq 1 .
$$

Proposition 6.6. $\mathcal{B}$ forms a Hopf super-algebra with respect to the counit

$$
\epsilon: \mathcal{B} \rightarrow \mathbb{k}, \quad \epsilon(p)=\epsilon_{\mathcal{R}(F)}(p(1))
$$

together with the coproduct and the antipode

$$
\Delta: \mathcal{B} \rightarrow \mathcal{B} \otimes \mathcal{B} \quad \text { and } \quad S: \mathcal{B} \rightarrow \mathcal{B}
$$

given by formulas analogous to (6.6) and (6.7).

To describe explicitly the formulas last mentioned, let

$$
F^{\mathrm{al}}=\operatorname{Sp}(\mathcal{R}(F))
$$

denote the affine group scheme represented by $\mathcal{R}(F)$; as in [6, p.1141] it may be called the universal algebraic hull of $F$. By definition $F^{\text {al }}$ is the functor which associates to each algebra $R$, the group $F^{\text {al }}(R)=\operatorname{Alg}(\mathcal{R}(F), R)$ of all algebra maps $\mathcal{R}(F) \rightarrow R$. This group scheme may not be algebraic, that is, $\mathcal{R}(F)$ may not be finitely generated. A right (resp., left) $F^{\text {al }}$ (super-) module is identified with a left (resp., right) $\mathcal{R}(F)$-(super-)comodule, and so by (5.9), with a right (resp., left) $F$-(super-)module. For example, the $\checkmark$ of the Harish-Chandra pair is a right $F^{\text {al }}$-module, on which each point $g \in F^{\mathrm{al}}(R)$ acts as the $R$-linear automorphism of $\mathrm{V} \otimes R$ determined by

$$
v \otimes 1 \mapsto v^{g}:=v^{(0)} \otimes g\left(v^{(-1)}\right),
$$

where we have used the notation (5.12). Let $p \in \mathcal{B}$. The value $\Delta(p)(a \otimes b)$ of the element $\Delta(p)$ in $\mathcal{B} \otimes \mathcal{B}=\operatorname{Hom}_{D \otimes D}(C \otimes C, \mathcal{R}(F) \otimes \mathcal{R}(F))$ at $a \otimes b \in C \otimes C$ is an element of the Hopf algebra $\mathcal{R}(F) \otimes \mathcal{R}(F)$ representing $F^{\text {al }} \times F^{\text {al }}$; it takes at every $R$-point $(g, h) \in F^{\text {al }}(R) \times F^{\text {al }}(R)$ the value

$$
\langle(g, h), \Delta(p)(a \otimes b)\rangle:=\left\langle g h, p\left(a^{h} b\right)\right\rangle,
$$

which is, more precisely, the value of $\left(p \otimes \operatorname{id}_{R}\right)\left(a^{h}(b \otimes 1)\right)$ at $g h \in F_{R}^{\mathrm{al}}(R)$. For $a \in C$ the element $S(p)(a)$ in $\mathcal{R}(F)$ tales at every $R$-point $g \in F^{\mathrm{al}}(R)$ the value

$$
\langle g, S(p)(a)\rangle:=\left\langle g^{-1}, p\left(S_{C}(a)^{g^{-1}}\right)\right\rangle,
$$

which is, more precisely, the value of $\left(p \otimes \operatorname{id}_{R}\right)\left(S_{C}(a)^{g^{-1}}\right)$ at $g^{-1} \in F_{R}^{\text {al }}(R)$.

The formulas (6.9) and (6.10) are seen to be quite similar to (6.6) and (6.7), respectively.

Proof of Proposition 6.6. The formulas (6.8)-(6.10) are the same as those given by [13, Lemma 4.14] as the structure maps of a Hopf super-algebra $\mathbf{A}$; see also [11, Lemma 26]. This construction of $\mathbf{A}$ can be done in a slightly more generalized situation, as is remarked by [13, Remark 4.21]. Proposition 6.6 follows from the last cited, generalized construction, with 
careful treatment. Indeed, we must be careful to apply those Lemma 4.14 and Remark 4.21 of [13], since there, the Hopf super-algebra is represented alternatively as

$$
\operatorname{Hom}_{\mathfrak{U}\left(\mathfrak{g}_{0}\right)}(\mathfrak{U}(\mathfrak{g}), \mathcal{R}(F)),
$$

where $\mathfrak{g}=P(C)$, if we modify the notation so as to fit with the present situation; on the other hand, in [11] it is represented essentially in the same way as our $\mathcal{B}$ is. (Working over a commutative ring in general, the article [13] must work with universal envelopes of Lie super-algebras. But, when working over a field of characteristic $\neq 2$ as we are doing, it is more natural to work with hyper-super-algebras.) Recall from Lemma 6.4 that $C$ is a Hopfalgebra object in SMod- $F$ including $D$ as a sub-object. One can restrict the structure to $\mathfrak{g}$, and then extend to $\mathfrak{U}(\mathfrak{g})$, so that $\mathfrak{g}$ turns into a Lie-algebra object, and $\mathfrak{U}(\mathfrak{g})$ turns into a Hopf-algebra object including $\mathfrak{U}\left(\mathfrak{g}_{0}\right)$ as a subobject. Note that the Hopf super-algebra map $\mathfrak{U}(\mathfrak{g}) \rightarrow C$ extending $\mathfrak{g} \subset C$ is a morphism in SMod- $F$ which maps $\mathfrak{U}\left(\mathfrak{g}_{0}\right)$ into $D$. Moreover, the supervector space given in (6.11) is identified with our $\mathcal{B}=\operatorname{Hom}(\wedge(\mathrm{V}), \mathcal{R}(F))$, since $\mathfrak{U}(\mathfrak{g}) \simeq \mathfrak{U}\left(\mathfrak{g}_{0}\right) \otimes \wedge(\vee)$ as left $\mathfrak{U}\left(\mathfrak{g}_{0}\right)$-super-module super-coalgebras. Then we can apply the cited results to prove the proposition; choose as the paring $\mathfrak{g}_{0} \times \mathcal{R}(F) \rightarrow \mathbb{k}$ required in [13, Remark 4.21], the restriction of the pairing $D \times \mathcal{R}(F) \rightarrow \mathbb{k}$ in (5.11).

Remark 6.7. We record here how we should modify [13, Remark 4.21], which was applied above, so as to completely fit in with our situation.

Suppose that the base field $\mathbb{k}$ is an arbitrary field of characteristic $\neq 2$. Let $\mathrm{F}$ be an affine group scheme represented by a Hopf algebra $O_{\mathrm{F}}$, and let $\mathfrak{C}$ be a hyper-super-algebra of finite type with $\mathfrak{D}=\underline{\mathfrak{C}}$. One sees that the same formulas as (6.8)-(6.10) construct a Hopf super-algebra on $\mathrm{Hom}_{\mathfrak{D}}\left(\mathfrak{C}, O_{\mathrm{F}}\right)$, if the following are satisfied (which are in fact modified from the assumptions posed in [13, Remark 4.21] in order to construct a Hopf super-algebra on $\left.\operatorname{Hom}_{\mathfrak{U}\left(\mathfrak{g}_{0}\right)}\left(\mathfrak{U}(\mathfrak{g}), O_{\mathrm{F}}\right)\right)$ :

(1) $\mathfrak{C}$ is a Hopf-algebra object in the category SMod- $\mathrm{F}$ of right F-supermodules; it then necessarily includes $\mathfrak{D}$ as a purely even sub-object;

(2) We are given a Hopf pairing $\langle\rangle:, \mathfrak{D} \times O_{\mathrm{F}} \rightarrow \mathbb{k}$ such that

(2a) $\left\langle a^{g}, p\right\rangle_{R}=\left\langle a,{ }^{g} p\right\rangle_{R}$ for all $a \in \mathfrak{D}, p \in O_{\mathrm{F}}$ and $g \in \mathrm{F}(R)$, where $R$ is an arbitrary algebra, and

(2b) through the pairing, the right F-action on $\mathfrak{D}$ or the associated

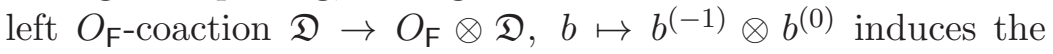
right adjoint action (see (5.19) $)$ in the sense that $\operatorname{ad}_{r}(a)(b)=$ $\left\langle a, b^{(-1)}\right\rangle b^{(0)}$ for all $a, b \in \mathfrak{D}$.

In $(2 \mathrm{a}),{ }^{g} p=p_{(2)} \otimes g\left(S\left(p_{(1)}\right) p_{(3)}\right)$ denotes the left F-adjoint action on $O_{\mathrm{F}}$, and $\langle,\rangle_{R}$ denotes the base extension of $\langle$,$\rangle to R$.

In our situation we see easily that the affine group scheme $F^{\text {al }}$ and the hyper-super-algebra $C$ satisfy the assumptions (1), (2) above, whence Proposition 6.6 follows. The Hopf pairing (5.11) is chosen as the one required by (2). We remark that $(2 \mathrm{a})$ is now reduced to

$$
\left\langle a^{g}, p\right\rangle=\left\langle a,{ }^{g} p\right\rangle, \quad a \in D, p \in \mathcal{R}(F), g \in F,
$$

which is satisfied by (6.3). 
Let us recall from [13, Section 4.4] (see also [11, Section 4.6]) how naturally the structure maps of $\mathcal{B}$ arise. The process will be used when we prove Proposition 6.5 and Theorem 6.18.

Step 1. Construction of $\mathcal{A}$ as a smash coproduct. Let $\mathrm{W}=\mathrm{V}^{*}$ denote the dual vector space of $\mathrm{V}$; these are both regarded as purely odd super-vector spaces. Let $\mathfrak{T}(\mathrm{V})=\bigoplus_{n>0} \mathfrak{T}^{n}(\mathrm{~V})$ denote the tensor algebra on $\checkmark$. This is an $\mathbb{N}$-graded Hopf super-algebra (see Section 2.2) in which every element in $\mathrm{V}$ is supposed to be an odd primitive; it is super-cocommutative, but is not super-commutative unless $\mathrm{V}=0$. The tensor coalgebra $\mathfrak{T}_{\mathrm{c}}(\mathrm{W})$ on $\mathrm{W}$ is the graded dual [21, Section 11.2]

$$
\mathfrak{T}_{\mathrm{c}}(\mathrm{W})=\bigoplus_{n \geq 0} \mathfrak{T}^{n}(\mathrm{~V})^{*}\left(=\bigoplus_{n \geq 0} \mathfrak{T}^{n}(\mathrm{~W})\right)
$$

of $\mathfrak{T}(\mathrm{V})$; it is, therefore, a super-commutative Hopf super-algebra.

The left $\mathcal{R}(F)$-coaction on $\mathrm{V}$ uniquely extends onto $\mathfrak{T}(\mathrm{V})$ so that $\mathfrak{T}(\mathrm{V})$ turns into an $\mathbb{N}$-graded Hopf-algebra object in $\mathcal{R}(F)$-SComod (or namely, an $\mathbb{N}$-graded Hopf super-algebra such that each $\mathbb{N}$-homogeneous component is a left $\mathcal{R}(F)$-comodule and the structure maps are all $\mathcal{R}(F)$-colinear). By duality $\mathfrak{T}_{\mathrm{c}}(\mathrm{W})$ turns into an $\mathbb{N}$-graded Hopf-algebra object in SComod- $\mathcal{R}(F)$. The associated smash coproduct [13, Page 3455, line-1] is denoted by

$$
\mathcal{A}=\mathcal{R}(F) \ltimes \mathfrak{T}_{\mathrm{c}}(\mathrm{W}) .
$$

This is a super-commutative $\mathbb{N}$-graded Hopf super-algebra, being the tensor product $\mathcal{R}(F) \otimes \mathfrak{T}_{\mathrm{c}}(\mathrm{W})$ as a super-algebra. The $n$-th component is $\mathcal{A}(n)=$ $\mathcal{R}(F) \otimes \mathfrak{T}^{n}(\mathrm{~W})$.

Step 2. Completion of $\mathcal{A}$. The projection $\mathcal{A} \rightarrow \mathcal{A}(0)=\mathcal{R}(F)$ is a Hopf super-algebra map, and so the kernel $\mathcal{A}^{+}:=\bigoplus_{n>0} \mathcal{A}(n)$ is a homogeneous Hopf super-ideal. Therefore, the $\mathcal{A}^{+}$-adic completion $\hat{\mathcal{A}}$ of $\mathcal{A}$ turns into a complete Hopf super-algebra. The last word is used in the wider sense as noted in Remark 3.4 (3). But this $\hat{\mathcal{A}}$ is just the direct product $\prod_{n \geq 0} \mathcal{R}(F) \otimes$ $\mathfrak{T}^{n}(\mathrm{~W})$, and $\hat{\mathcal{A}} \hat{\otimes} \hat{\mathcal{A}}=\prod_{m, n \geq 0} \mathcal{R}(F) \otimes \mathfrak{T}^{m}(\mathrm{~W}) \otimes \mathcal{R}(F) \otimes \mathfrak{T}^{n}(\mathrm{~W})$; the structure maps

$$
\hat{\Delta}: \hat{\mathcal{A}} \rightarrow \hat{\mathcal{A}} \hat{\otimes} \hat{\mathcal{A}}, \quad \hat{\epsilon}: \hat{\mathcal{A}} \rightarrow \mathbb{k}, \quad \hat{S}: \hat{\mathcal{A}} \rightarrow \hat{\mathcal{A}}
$$

only extend those of $\mathcal{A}$ to the relevant direct products in the obvious manner.

Remark 6.8. The right $F$-action on $\mathrm{V}$ uniquely extends onto $\mathfrak{T}(\mathrm{V})$ so that $\mathfrak{T}(\mathrm{V})$ turns into an $\mathbb{N}$-graded Hopf-algebra object in SMod- $F$. The associated smash product $F \ltimes \mathfrak{T}(\mathrm{V})$ is a (not necessarily super-commutative) Hopf super-algebra, in which every element of $F$ is supposed to be grouplike. The dual $(F \ltimes \mathfrak{T}(\mathrm{V}))^{*}$ is a complete Hopf super-algebra in the wider sense; see Remark 3.4 (3), again. See also Proposition 2.3 for the complete topological dual (super-)coalgebra structure. The natural pairings $\left(F \times \mathfrak{T}^{n}(\mathrm{~V})\right) \times\left(\mathcal{R}(F) \otimes \mathfrak{T}^{n}(\mathrm{~W})\right) \rightarrow \mathbb{k}, n \geq 0$, amount to $(F \ltimes \mathfrak{T}(\mathrm{V})) \times \hat{\mathcal{A}} \rightarrow \mathbb{k}$, which induces a continuous injection $\hat{\mathcal{A}} \rightarrow(F \ltimes \mathfrak{T}(\mathrm{V}))^{*}$ of Hopf superalgebras, as is seen from the construction above. 
Step 3. Transfer of the completed structure. Note from Lemma 5.11 that $\mathfrak{T}(\mathrm{V})$ is an $\mathbb{N}$-graded Hopf-algebra object in $\mathrm{SMod}-D$, as well. The associated smash product

$$
\mathcal{C}:=D \ltimes \mathfrak{T}(\mathrm{V}) .
$$

is a (not necessarily super-commutative) $\mathbb{N}$-graded Hopf super-algebra, being the tensor product $D \otimes \mathfrak{T}(\mathrm{V})$ as a super-coalgebra. The $n$-th component is $\mathcal{C}(n)=D \otimes \mathfrak{T}^{n}(\mathrm{~V})$. Note that $\mathcal{C}$ is a Hopf-algebra object in SMod- $F$, just as $C$ is by Lemma 6.4. We have the natural identification

$$
\hat{\mathcal{A}}=\operatorname{Hom}_{D}(\mathcal{C}, \mathcal{R}(F))(=\operatorname{Hom}(\mathfrak{T}(\mathrm{V}), \mathcal{R}(F))
$$

and, more generally,

$$
\hat{\mathcal{A}}^{\hat{\otimes} n}=\operatorname{Hom}_{D^{\otimes n}}\left(\mathcal{C}^{\otimes n}, \mathcal{R}(F)^{\otimes n}\right), \quad n \geq 1 .
$$

Transfer the structure maps (6.13) of $\hat{\mathcal{A}}$ to $\operatorname{Hom}_{D}(\mathcal{C}, \mathcal{R}(F)$ ). As was proved by [13, Proposition 4.11], the resulting maps are given by the formulas (6.8)(6.10) modified so that $a, b \in \mathcal{C}, p \in \operatorname{Hom}_{D}(\mathcal{C}, \mathcal{R}(F))$, and $S_{C}$ is replaced with $S_{\mathcal{C}}$.

Step 4. Restriction of the transferred structure. Note that $C$ is a quotient of $\mathcal{C}$, as a Hopf-algebra object in SMod- $F$ including $D$ as a subobject. We then see that $\hat{\mathcal{A}}^{\otimes n}$ naturally includes $\mathcal{B}^{\otimes n}=\operatorname{Hom}_{D \otimes n}\left(C^{\otimes n}\right.$, $\left.\mathcal{R}(F)^{\otimes n}\right)$. Moreover, the transferred structure maps of $\operatorname{Hom}_{D}(\mathcal{C}, \mathcal{R}(F))$ restrict to those of $\mathcal{B}$ given by (6.8)-(6.10), which, therefore, satisfy the Hopfalgebra axioms. Thus $\mathcal{B}$ turns into a Hopf super-algebra; see [13, Lemma 4.14].

Proof of Proposition [6.5. Given open sets $U \subset|F| \times|F|, V \subset|F|$ with $\mu(U) \subset V$, we have the push-outs

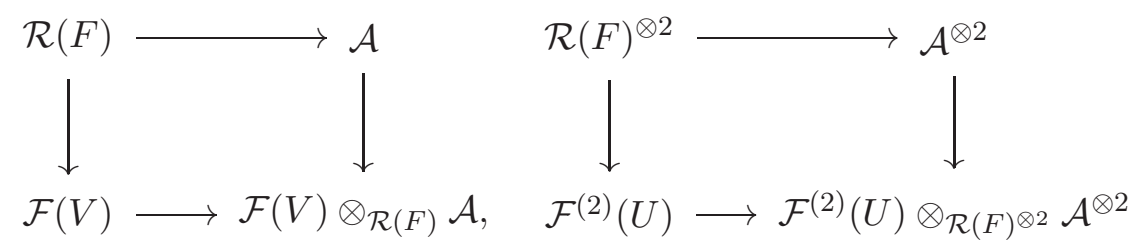

of super-algebras, where the vertical arrows on the left-hand side are the inclusions $\mathcal{R}(F)^{\otimes n} \hookrightarrow \mathcal{F}(|F|)^{\otimes n} \subset \mathcal{F}^{(n)}\left(|F|^{n}\right), n=1,2$, followed by the restriction maps. The two tensor products are identified with $\mathcal{F}(V) \otimes \mathfrak{T}_{\mathrm{c}}(\mathrm{W})$ and $\mathcal{F}^{(2)}(U) \otimes \mathfrak{T}_{\mathrm{c}}(\mathrm{W}) \otimes \mathfrak{T}_{\mathrm{c}}(\mathrm{W})$, respectively. Since the coproducts $\Delta_{\mathcal{R}(F)}$ of $\mathcal{R}(F)$ and $\Delta_{\mathcal{A}}$ of $\mathcal{A}$ and the sheaf-morphism $\mu^{*}: \mathcal{F}(V) \rightarrow \mathcal{F}^{(2)}(U)$ associated with the product $\mu$ on $F$ are compatible with the relevant arrows, we have uniquely a map

$$
\Delta^{\prime}: \mathcal{F}(V) \otimes \mathfrak{T}_{\mathrm{c}}(\mathrm{W}) \rightarrow \mathcal{F}^{(2)}(U) \otimes \mathfrak{T}_{\mathrm{c}}(\mathrm{W}) \otimes \mathfrak{T}_{\mathrm{c}}(\mathrm{W})
$$

which, together with the preceding three, constitutes a map between the two push-outs. By completion (to direct products) followed by an identification just as for $\hat{\mathcal{A}}$, it derives

$$
\hat{\Delta}^{\prime}: \operatorname{Hom}_{D}(\mathcal{C}, \mathcal{F}(V)) \rightarrow \operatorname{Hom}_{D^{\otimes 2}}\left(\mathcal{C}^{\otimes 2}, \mathcal{F}^{(2)}(U)\right) .
$$


Similarly, given open sets $U, V \subset|F|$ with $\iota(U) \subset V$, we have the push-out

$$
S^{\prime}: \mathcal{F}(V) \otimes \mathfrak{T}_{\mathrm{c}}(\mathrm{W}) \rightarrow \mathcal{F}(U) \otimes \mathfrak{T}_{\mathrm{c}}(\mathrm{W})
$$

of $\iota^{*}$ and the antipode $S_{\mathcal{A}}$ of $\mathcal{A}$ over the antipode $S_{\mathcal{R}(F)}$ of $\mathcal{R}(F)$, which derives

$$
\hat{S}^{\prime}: \operatorname{Hom}_{D}(\mathcal{C}, \mathcal{F}(V)) \rightarrow \operatorname{Hom}_{D}(\mathcal{C}, \mathcal{F}(U)) .
$$

We have the sheaf of super-algebras on $|F|$

$$
\operatorname{Hom}_{D}(\mathcal{C}, \mathcal{F})
$$

which associates $\operatorname{Hom}_{D}(\mathcal{C}, \mathcal{F}(U))$ to every open set $U \subset|F|$. For $n>0$ the sheaves $\operatorname{Hom}_{D^{\otimes n}}\left(\mathcal{C}^{\otimes n}, \mathcal{F}^{(n)}\right)$ on $|F|^{n}$ are analogously defined. One sees that the $\hat{\Delta}^{\prime}$ and $\hat{S}^{\prime}$ above give rise to sheaf-morphisms $\hat{\Delta}^{\prime}: \operatorname{Hom}_{D}(\mathcal{C}, \mathcal{F}) \rightarrow$ $\mu_{*} \operatorname{Hom}_{D^{\otimes 2}}\left(\mathcal{C}^{\otimes 2}, \mathcal{F}^{(2)}\right)$ and $\hat{S}^{\prime}: \operatorname{Hom}_{D}(\mathcal{C}, \mathcal{F}) \rightarrow \iota_{*} \operatorname{Hom}_{D}(\mathcal{C}, \mathcal{F})$. These are presented by formulas analogous to (6.6) and (6.7), just as the structure maps of $\hat{\mathcal{A}}$ are given by formulas analogous to (6.8)- 6 (6.10).

Recall from Step 4 above that $C$ is a quotient of $\mathcal{C}$ as a Hopf-algebra object in SMod- $F$ including $D$. One then sees that $\mathcal{P}$ is a sub-sheaf of the sheaf $\operatorname{Hom}_{D}(\mathcal{C}, \mathcal{F})$, and the sheaf-morphisms $\hat{\Delta}^{\prime}$ and $\hat{S}^{\prime}$ for the last sheaf restrict to the sheaf-morphisms $\Delta$ and $S$ for $\mathcal{P}$ which are presented by (6.6) and (6.7).

In view of Proposition 4.8 it remains to prove, passing to the completed stalks, that

$$
\hat{\Delta}_{g, h}: \hat{\mathcal{P}}_{g h} \rightarrow \hat{\mathcal{P}}_{g} \hat{\otimes} \hat{\mathcal{P}}_{h}, \quad \hat{S}_{g}: \hat{\mathcal{P}}_{g^{-1}} \rightarrow \hat{\mathcal{P}}_{g}, \quad g, h \in|F|
$$

satisfy the axioms of coalgebra and of antipode. For example, the coassociativity means $\hat{\Delta}_{g, h} \hat{\otimes} \operatorname{id}_{\hat{\mathcal{P}}_{\ell}}=\mathrm{id}_{\hat{\mathcal{P}}_{g}} \hat{\otimes} \hat{\Delta}_{h, \ell}$, and the left counit-property means that the composite

$$
\hat{\mathcal{P}}_{g} \stackrel{\hat{\Delta}_{e, g}}{\longrightarrow} \hat{\mathcal{P}}_{e} \hat{\otimes} \hat{\mathcal{P}}_{g} \stackrel{\hat{\epsilon}_{e} \hat{\otimes} \mathrm{id}}{\longrightarrow} \mathbb{k} \hat{\otimes} \hat{\mathcal{P}}_{g}=\hat{\mathcal{P}}_{g}
$$

coincides with the identity, where $\hat{\epsilon}_{e}: \hat{\mathcal{P}}_{e} \rightarrow \mathbb{k}$ denotes the projection to the residue field; see (5.2). But one sees from the argument of the last paragraph that it suffices to prove the analogous assertions for the sheaf $\operatorname{Hom}_{D}(\mathcal{C}, \mathcal{F})$, or even for the sheaf $\mathcal{F} \otimes \mathfrak{T}_{\mathrm{c}}(\mathrm{W})$ which associates $\mathcal{F}(U) \otimes \mathfrak{T}_{\mathrm{c}}(\mathrm{W})$ to every open set $U \subset|F|$. To be explicit the last mentioned, analogous assertion is the following: the unique maps $\hat{\mathcal{F}}_{g h} \otimes \mathfrak{T}_{\mathrm{c}}(\mathrm{W}) \rightarrow \hat{\mathcal{F}}_{g} \hat{\otimes} \hat{\mathcal{F}}_{h} \otimes \mathfrak{T}_{\mathrm{c}}(\mathrm{W}) \otimes \mathfrak{T}_{\mathrm{c}}(\mathrm{W})$ and $\hat{\mathcal{F}}_{g^{-1}} \otimes \mathfrak{T}_{\mathrm{c}}(\mathrm{W}) \rightarrow \hat{\mathcal{F}}_{g} \otimes \mathfrak{T}_{\mathrm{c}}(\mathrm{W})$ that together with

$\widehat{\mu}^{*}{ }_{g, h}: \hat{\mathcal{F}}_{g h} \rightarrow \hat{\mathcal{F}}_{g} \hat{\otimes} \hat{\mathcal{F}}_{h}, \quad \Delta_{\mathcal{A}}: \mathcal{A} \rightarrow \mathcal{A} \otimes \mathcal{A}, \quad \Delta_{\mathcal{R}(F)}: \mathcal{R}(F) \rightarrow \mathcal{R}(F) \otimes \mathcal{R}(F)$, and respectively with

$$
\widehat{\iota}^{*}: \hat{\mathcal{F}}_{g^{-1}} \rightarrow \hat{\mathcal{F}}_{g}, \quad S_{\mathcal{A}}: \mathcal{A} \rightarrow \mathcal{A}, \quad S_{\mathcal{R}(F)}: \mathcal{R}(F) \rightarrow \mathcal{R}(F)
$$

constitute maps of push-outs satisfy the axioms of coalgebra and of antipode. Indeed, the assertion is true since the displayed six maps satisfy the axioms. Note that as the counit on $\hat{\mathcal{F}}_{e} \otimes \mathfrak{T}_{\mathrm{c}}(\mathrm{W})$ one should choose the tensor product $\hat{\epsilon}_{e} \otimes \epsilon_{0}: \hat{\mathcal{F}}_{e} \otimes \mathfrak{T}_{\mathrm{c}}(\mathrm{W}) \rightarrow \mathbb{k} \otimes \mathbb{k}=\mathbb{k}$, where $\epsilon_{0}: \mathfrak{T}_{\mathrm{c}}(\mathrm{W}) \rightarrow \mathfrak{T}^{0}(\mathrm{~W})=\mathbb{k}$ denotes the projection; it constitutes a map of push-outs, together with $\hat{\epsilon}_{e}: \hat{\mathcal{F}}_{e} \rightarrow \mathbb{k}$, $\epsilon_{\mathcal{A}}: \mathcal{A} \rightarrow \mathbb{k}$ and $\epsilon_{\mathcal{R}(F)}: \mathcal{R}(F) \rightarrow \mathbb{k}$. 
Lemma 6.9. Let $\Psi(F, \mathrm{~V})$ denote the super Lie group $(|F|, \mathcal{P})$ which is thus constructed from a Harish-Chandra pair $(F, \mathrm{~V})$. The assignment gives rise to a functor

$$
\Psi: \mathrm{HCP} \rightarrow \mathrm{SLG}, \Psi(F, \mathrm{~V})=(|F|, \mathcal{P}) .
$$

Proof. With the notation above one sees easily that the constructions of

(i) the inclusion $C \supset D$ of Hopf-algebra objects in $\mathrm{SMod}-F$, and

(ii) the sheaf $\mathcal{F}$ of left $D$-module algebras

from $(F, \vee)$ are both functorial. This implies that $\Psi$ is functorial.

Proof of Theorem 6.3 (First half). We shall prove that $\Psi$ is a quasi-inverse of $\Phi$. But our aim here is only to prove $\Phi \circ \Psi \simeq$ id. The following subsection is devoted to proving $\Psi \circ \Phi \simeq$ id.

Given $(F, \mathrm{~V}) \in \mathrm{HCP}$, let $C$ and $G:=(|F|, \mathcal{P})$ be the hyper-super-algebra and the super Lie group, respectively, constructed as above. For every open set $U \subset|F|$ we have $\mathcal{P}_{\text {red }}(U)=\operatorname{Hom}_{D}(D, \mathcal{F}(U))=\mathcal{F}(U)$ so that $G_{\text {red }}=$ $F(=(|F|, \mathcal{F}))$. It remains to show that the right $F$-module $\mathrm{V}$ and the bracket [, ] of the Harish-Chandra pair recover from $G$. The former $\mathrm{V}$ appears as the odd component $P(C)_{1}$ of $P(C)$; recall from Lemma 6.4 that $F$ acts on $C$ from the right, whence $P(C)$ and $P(C)_{1}$ are right $F$-modules by restriction. In view of (6.1) it suffices to prove that there is a natural $F$-equivariant isomorphism $C \stackrel{\simeq}{\longrightarrow}$ hy $(G)$ which is identical on $D=\operatorname{hy}(F)$, or equivalently, there is such an isomorphism $\hat{\mathcal{P}}_{e} \stackrel{\simeq}{\longrightarrow} C^{*}$ which is compatible with the natural maps to $\hat{\mathcal{F}}_{e}$. Recall from (6.5) and Proposition 5.17 that $\hat{\mathcal{P}}_{e}=\operatorname{Hom}_{D}\left(C, \hat{\mathcal{F}}_{e}\right)$, and it is equipped with such a left $F$-action that induces the right $F$-adjoint action on hy $(G)$; see also Remark 5.18.

Let $\hat{\Delta}_{g^{-1}, h, g}^{(2)}: \hat{\mathcal{P}}_{g^{-1} h g} \rightarrow \hat{\mathcal{P}}_{g^{-1}} \hat{\otimes} \hat{\mathcal{P}}_{h} \hat{\otimes} \hat{\mathcal{P}}_{g}$ represent (id $\left.\hat{\otimes} \hat{\Delta}_{h, g}\right) \circ \hat{\Delta}_{g^{-1}, h g}(=$ $\left.\left(\hat{\Delta}_{g^{-1}, h} \hat{\otimes} \mathrm{id}\right) \circ \hat{\Delta}_{g^{-1} h, g}\right)$. Then we see from (6.6) that

$$
\left(\hat{\epsilon}_{g^{-1}} \hat{\otimes} \hat{\epsilon}_{h} \hat{\otimes} \hat{\epsilon}_{g}\right)\left(\hat{\Delta}_{g^{-1}, h, g}^{(2)}(p)(a \otimes b \otimes c)\right)=\left\langle g^{-1} h g, p\left(a^{h g} b^{g} c\right)\right\rangle,
$$

where $g, h \in F, p \in \hat{\mathcal{P}}_{g^{-1} h g}$ and $a, b, c \in C$. (We remark that to be precise, the $p$ on the right-hand side should read an element of some/any $\mathcal{P}(U)$ with $g^{-1} h g \in U$, whose germ is the $p \in \hat{\mathcal{P}}_{g^{-1} h g}$.) It follows that the $F$-action on $\hat{\mathcal{P}}_{e}$ is given by

$$
\left.{ }^{g} p\right)(b)={ }^{g}\left(p\left(b^{g}\right)\right), \quad g \in F, p \in \hat{\mathcal{P}}_{e}, b \in C .
$$

Define $\xi: \hat{\mathcal{P}}_{e} \rightarrow C^{*}$ by

$$
\xi(p)(a)=\hat{\epsilon}_{e}(p(a)), \quad p \in \hat{\mathcal{P}}_{e}, a \in C .
$$

This $\xi$ is seen to be $F$-equivariant. Since one sees that $\hat{\mathcal{F}}_{e}=D^{*}$ as a left $D$-module algebra, it follows that $\xi$ is a natural super-algebra isomorphism with the desired compatibility; indeed, it is identified with the canonical isomorphism $\operatorname{Hom}_{D}\left(C, D^{*}\right) \simeq C^{*}$. Moreover, it preserves the coproduct since $(\xi \hat{\otimes} \xi) \circ \hat{\Delta}_{e, e}(p)(a \otimes b)$ coincides with the value $\Delta(p)(a \otimes b)(e, e)=$ $\langle e, p(a b)\rangle$ given by (6.6), where $p \in \hat{\mathcal{P}}_{e}$ and $a, b \in C$. (To be precise the same remark as above should apply to the $p$ in the last equation.) 
6.3. Completion of the proof. Let $G=(|G|, \mathcal{O}) \in \mathrm{SLG}$, and set

$$
(F, \mathrm{~V})=\Phi(G) \in \mathrm{HCP},
$$

or explicitly, $(F, \mathrm{~V})=\left(G_{\text {red }}, \operatorname{Lie}(G)_{1}\right)$. Let $\mathcal{F}=\mathcal{O}_{\text {red }}$ be the structure sheaf of the Lie group $F=G_{\text {red }}$, and set $D=\operatorname{hy}(F)(=$ hy $(G))$.

Let $C$ denote the hyper-super-algebra constructed from $(F, \mathrm{~V})$ as in the preceding subsection; see (6.1), 6.2).

Lemma 6.10. Recall from Lemma 6.4 and Proposition 5.17 (2) that the hyper-super-algebras $C$ and hy $(G)$ are both Hopf-algebra objects in SMod-F. These Hopf-algebra objects are naturally identified.

Proof. By (6.1) the inclusions $D=\operatorname{hy}(G) \hookrightarrow \operatorname{hy}(G) \hookleftarrow \operatorname{Lie}(G)_{1}=\mathrm{V}$ amount

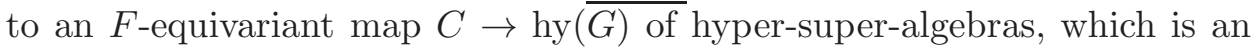
isomorphism by [10, Theorem 3.6]; see also (3.10).

By Lemma 6.10 we have $C=\left(\hat{\mathcal{O}}_{e}\right)^{\star}$. The resulting canonical pairing will be used in the proof below, denoted by $\langle\rangle:, C \times \hat{\mathcal{O}}_{e} \rightarrow \mathbb{k}$; it satisfies

$$
\left\langle a^{g}, p\right\rangle=\left\langle a,{ }^{g} p\right\rangle, \quad a \in C, p \in \hat{\mathcal{O}}_{e}, g \in F .
$$

Concerning the super Lie group $\Psi(F, \mathrm{~V})$ the associated Lie group is $F=$ $G_{\text {red }}$. Let $\mathcal{P}$ be the structure sheaf given in (6.4), and so let

$$
(|F|, \mathcal{P})=\Psi(F, \mathrm{~V}) \in \mathrm{SLG} .
$$

In what follows we let red : $\mathcal{O} \rightarrow \mathcal{O}_{\text {red }}=\mathcal{F}$ denote the natural sheafepimorphism. Consistent with this is the symbol $\widehat{\operatorname{red}}_{g}: \hat{\mathcal{O}}_{g} \rightarrow \hat{\mathcal{F}}_{g}$ which was used (see (5.17)) and will be soon used to denote the natural projection.

Proposition 6.11. We have the following.

(1) For every open set $U \subset|G|$, define

$$
\eta_{U}(p)(a)=\operatorname{red}_{U}(a \triangleright p), \quad p \in \mathcal{O}(U), a \in C(=\operatorname{hy}(G)),
$$

where $a \triangleright p$ indicates the hy $(G)$-action on $\mathcal{O}$ given by Proposition 5.14. Then this indeed defines a morphism $\eta: \mathcal{O} \rightarrow \mathcal{P}$ of sheaves of superalgebras.

(2) (id, $\eta):(|F|, \mathcal{P}) \rightarrow(|G|, \mathcal{O})=G$ is an isomorphism of super Lie groups.

Proof. (1) This is easy to see.

For the proof of Part 2 below we remark that for every $g \in|F|(=|G|)$, $\hat{\eta}_{g}: \hat{\mathcal{O}}_{g} \rightarrow \hat{\mathcal{P}}_{g}=\operatorname{Hom}_{D}\left(C, \hat{\mathcal{F}}_{g}\right)$ is given by

$$
\hat{\eta}_{g}(p)(a)=\left(\widehat{\operatorname{red}}_{g} \hat{\otimes} a\right) \circ \hat{\Delta}_{g, e}(p), \quad p \in \hat{\mathcal{O}}_{g}, a \in C .
$$

This formula is expressed by using the sigma notation $\hat{\Delta}_{g, e}(p)=p_{(1)} \otimes p_{(2)}$, so as

$$
\hat{\eta}_{g}(p)(a)=\widehat{\operatorname{red}}_{g}\left(p_{(1)}\right)\left\langle a, p_{(2)}\right\rangle .
$$

Note that the sum on the right-hand side above is finite since $p_{(1)} \otimes p_{(2)}$ may be regarded as an element of some ordinary tensor product $\hat{\mathcal{O}}_{g} \otimes\left(\hat{\mathcal{O}}_{e} / \hat{\mathfrak{m}}_{e}^{r}\right)$, where $r>0$ is an integer such that $\left\langle a, \hat{\mathfrak{m}}_{e}^{r}\right\rangle=0$.

(2) Let us prove that $(\mathrm{id}, \eta)$ is a morphism in SLG, using the notation of Section 5.1. Let $\eta^{(2)}: \mathcal{O}^{(2)}=\mathcal{O}_{G \times G} \rightarrow \mathcal{P}^{(2)}$ denote the sheaf-morphism 
associated with (id, $\eta) \times($ id, $\eta)$. In view of Remark 5.5 (2) we have to prove that for an open set $\emptyset \neq U \subset|G|$,

$$
\eta^{(2)}(\Delta(p))(a \otimes b)(g, h)=\eta(p)\left(a^{h} b\right)(g h),
$$

where $p \in \mathcal{O}(U), a, b \in C$, and $(g, h) \in \mu^{-1}(U)$ (or $g h \in U$ ). If we let $q \in \hat{\mathcal{O}}_{g h}$ denote the germ given by $p$, it suffices in view of Remark 4.7 (2) to prove

$$
\left(\hat{\epsilon}_{g} \hat{\otimes} \hat{\epsilon}_{h}\right)\left(\left(\hat{\eta}_{g} \hat{\otimes} \hat{\eta}_{h}\right) \circ \hat{\Delta}_{g, h}(q)(a \otimes b)\right)=\hat{\epsilon}_{g h}\left(\hat{\eta}_{g h}(q)\left(a^{h} b\right)\right) .
$$

To verify this we use the sigma notation, such as used in (6.17) above,

$$
\hat{\Delta}_{g_{1}, \ldots, g_{s}}(q)=q_{(1)} \otimes \cdots \otimes q_{(s)}, \quad g_{1} \ldots g_{s}=g h .
$$

This is justified by the coassociativity of $\hat{\Delta}$; see the proof of Proposition 6.5. Again in this situation the value may be supposed to be in some ordinary tensor product so that the relevant sum is finite. The verification is done, using (6.16), so as

$$
\begin{aligned}
\mathrm{LHS} & =\hat{\epsilon}_{g}\left(q_{(1)}\right)\left\langle a, q_{(2)}\right\rangle \hat{\epsilon}_{h}\left(q_{(3)}\right)\left\langle b, q_{(4)}\right\rangle \\
& =\hat{\epsilon}_{g}\left(q_{(1)}\right) \hat{\epsilon}_{h}\left(q_{(2)}\right) \hat{\epsilon}_{h^{-1}}\left(q_{(3)}\right)\left\langle a, q_{(4)}\right\rangle \hat{\epsilon}_{h}\left(q_{(5)}\right)\left\langle b, q_{(6)}\right\rangle \\
& =\hat{\epsilon}_{g}\left(q_{(1)}\right) \hat{\epsilon}_{h}\left(q_{(2)}\right)\left\langle a,{ }^{h} q_{(3)}\right\rangle\left\langle b, q_{(4)}\right\rangle \\
& =\hat{\epsilon}_{g h}\left(q_{(1)}\right)\left\langle a^{h}, q_{(2)}\right\rangle\left\langle b, q_{(3)}\right\rangle=\mathrm{RHS} .
\end{aligned}
$$

To complete the proof we will see by applying Lemma 5.9 that $\phi:=(\mathrm{id}, \eta)$ is an isomorphism. Obviously, $\phi_{\text {red }}: F \rightarrow F=G_{\text {red }}$ is the identity map. It remains to verify that $\operatorname{Lie}(\phi)_{1}$ is an isomorphism. Let $\mathrm{W}=\mathrm{V}^{*}$, as before. One sees from

$$
\epsilon_{e}(v \triangleright q)=\langle v, q\rangle, \quad v \in \mathrm{V}, q \in \mathcal{O}_{e}
$$

that the map $\mathcal{O}_{e} \rightarrow \mathrm{W}, q \mapsto \epsilon_{e}\left(\left.\eta_{e}(q)\right|_{\mathrm{V}}\right)$ coincides with the natural projection $\mathcal{O}_{e} \rightarrow\left(\mathfrak{m}_{e} / \mathfrak{m}_{e}^{2}\right)_{1}=\mathrm{W}$. This implies that $\operatorname{Lie}(\phi)_{1}$ is the identity map.

Obviously, $\eta$ is natural in $G$. This completes the proof of Theorem 6.3.

Remark 6.12. The sheaf-isomorphism $\eta$ above looks like the $\eta^{*}$ given by [1, Page 133, lines 2-3] and [2, Page 41, lines 17-18] in the $C^{\infty}$ - and the complex analytic situations. An essential difference is in that the $\eta^{*}$ contains the sign $(-1)^{|X|}$ in its expression. But the sign is dispensable, since obviously, one remains to have a desired isomorphism after removing it.

6.4. Consequences of the category equivalence. Here we have two corollaries to Theorem 6.3. The first one is the following.

Corollary 6.13. Every super Lie group is s-split as a super-manifold; see Definition 4.1 (2).

Proof. By the theorem every super Lie group is of the form $(|F|, \mathcal{P})$, whence it is s-split since $\mathcal{P}=\mathcal{F} \otimes \wedge(\mathrm{W})$; see (6.4).

To state the second one, we need some preliminaries. Assume for a while that the base field $\mathbb{k}$ is an arbitrary infinite field (e.g. a complete field), and let $\overline{\mathbb{k}}$ denote the algebraic closure of $\mathbb{k}$. Let $\mathrm{F}$ be an affine algebraic group scheme $\mathrm{F}$ (over $\mathbb{k}$ ) represented by $O_{\mathrm{F}}$. We say that $\mathrm{F}$ is an algebraic 
matrix group [26, Sections 4.4-4.5], if the following equivalent conditions are satisfied:

(i) The intersection of the maximal ideals of $O_{\mathrm{F}}$ of codimension 1 is zero;

(ii) $O_{\mathrm{F}}$ is reduced, and the group $\mathrm{F}(\mathbb{k})$ of $\mathbb{k}$-points is Zariski dense in $\mathrm{F}(\overline{\mathbb{k}})$ or equivalently, Zariski dense in $\operatorname{Spec}\left(O_{\mathrm{F}}\right)$;

(iii) The algebra map $O_{\mathrm{F}} \rightarrow \operatorname{Map}(\mathrm{F}(\mathbb{k}), \mathbb{k})$ which associates to $p \in O_{\mathrm{F}}$, the map $\mathrm{F}(\mathbb{k}) \rightarrow \mathbb{k}, g \mapsto g(p)$ is an injection.

The image of the algebra map in (iii) is denoted by $\operatorname{Poly}(F(\mathbb{k}), \mathbb{k})$, whose elements are called polynomial functions. Roughly speaking, an algebraic matrix group is an affine algebraic group scheme which arises from a Zariski closed matrix group. If $\mathrm{F}$ is an algebraic matrix group, then we have $O_{\mathrm{F}} \otimes \overline{\mathbb{k}} \simeq$ $\operatorname{Poly}(\mathrm{F}(\overline{\mathbb{k}}), \overline{\mathbb{k}})$, whence $O_{\mathrm{F}} \otimes \overline{\mathbb{k}}$ is reduced, or equivalently, $O_{\mathrm{F}}($ or $\mathrm{F})$ is smooth. In addition, any element of $O_{\mathrm{F}} \otimes \overline{\mathbb{k}}$ that takes values in $\mathbb{k}$ on $\mathrm{F}(\mathbb{k})$ is contained in $O_{\mathrm{F}}$; this, applied to idempotents, shows that the largest finite-dimensional separable subalgebra $\pi_{0}\left(O_{\mathrm{F}}\right)$ of $O_{\mathrm{F}}$ (see [26, Section 6.5]) is spanned by idempotents. If $\mathbb{k}$ is algebraically closed, an algebraic matrix group is the same as a smooth affine algebraic group scheme. If in addition, char $\mathbb{k}=0$, then every affine algebraic group scheme is smooth, and is, therefore, an algebraic matrix group.

Assume char $\mathbb{k} \neq 2$, and let ASG denote the category of affine algebraic super-group schemes. Suppose $\mathrm{G} \in \mathrm{ASG}$. Let $O_{\mathrm{G}}$ denote the finitely generated Hopf super-algebra which represents $\mathrm{G}$, and let $\mathfrak{m}_{\mathrm{G}}=\operatorname{Ker} \epsilon$ denote the kernel of the counit of $O_{\mathrm{G}}$. The hyper-super-algebra hy $(\mathrm{G})$ of $\mathrm{G}$ is the Hopf super-subalgebra

$$
\operatorname{hy}(\mathrm{G})=\bigcup_{n>0}\left(O_{\mathrm{G}} / \mathfrak{m}_{\mathrm{G}}^{n}\right)^{*}
$$

of the dual Hopf super-algebra $O_{\mathrm{G}}^{\circ}$ of $O_{\mathrm{G}}$; the dual hy $(\mathrm{G})^{*}$ of this hy $(\mathrm{G})$ is the $\mathfrak{m}_{\mathrm{G}}$-adic completion $\widehat{O_{\mathrm{G}}}$ of $O_{\mathrm{G}}$, or equally, the completion of the localized super-algebra $\left(O_{\mathrm{G}}\right)_{\mathfrak{m}_{\mathrm{G}}}$. The Lie super-algebra Lie $(\mathrm{G})$ of $\mathrm{G}$ is $P($ hy $(\mathrm{G}))$. The constructions of hy $(\mathrm{G})$ and of $\operatorname{Lie}(\mathrm{G})$ are functorial; cf. Proposition 5.7 .

As was touched before, an algebraic counterpart of Theorem 6.3 (see [11, Theorem 29], [12, Theorem 6.5]) shows a category equivalence between ASG and the category of algebraic Harish-Chandra pairs. Such a pair (F,V) consists of an affine algebraic group scheme $F$, a finite-dimensional $F$-module $\mathrm{V}$ and an F-equivariant linear map $[]:, \mathrm{V} \otimes \mathrm{V} \rightarrow \operatorname{Lie}(\mathrm{F})$ which satisfy the same formulas as (P1) and (P2) in Definition 6.1, Lie(F) is regarded as a right $\mathrm{F}$-module by the right adjoint action (induced from the right adjoint F-action on $\mathrm{F}$ ).

Let $G \in A S G$, and suppose that it corresponds to an algebraic HarishChandra pair $(F, V)$. The affine algebraic group scheme $F$ is the group-valued functor obtained from $\mathrm{G}$, restricting the domain to the category of algebras, and it is represented by $\overline{O_{\mathrm{G}}}=O_{\mathrm{G}} /\left(\left(O_{\mathrm{G}}\right)_{1}\right)$; see (2.2). We say that $\mathrm{G}$ is smooth if $O_{\mathrm{G}}$ is smooth. This is equivalent to saying that each/one of $\mathrm{F}$, hy $(F)$ and hy $(G)$ is smooth (see [16, Proposition A.3]), and the equivalent conditions are necessarily satisfied if char $\mathbb{k}=0$. We may say that $G$ is connected, precisely when $\mathrm{F}$ is connected. 
Definition 6.14. We say that $G$ is an algebraic matrix super-group, if the intersection of the maximal super-ideals in $O_{\mathrm{G}}$ of codimension 1 is zero, or equivalently, if $F$ is an algebraic matrix group. We let AMSG denote the full subcategory of ASG consisting of all algebraic matrix super-groups.

Every algebraic matrix super-group is smooth. If $\mathbb{k}$ is an algebraically closed field of characteristic zero, we have AMSG $=$ ASG .

Let us return to the situation that $\mathbb{k}$ is a complete field of characteristic $\neq 2$. The second corollary to Theorem 6.3 is the following.

Corollary 6.15. We have a natural functor

$$
\mathrm{AMSG} \rightarrow \mathrm{SLG}, \quad \mathrm{G} \mapsto \mathrm{G}^{\text {an }},
$$

such that

$$
\operatorname{hy}(\mathrm{G})=\operatorname{hy}\left(\mathrm{G}^{\text {an }}\right), \quad \operatorname{Lie}(\mathrm{G})=\operatorname{Lie}\left(\mathrm{G}^{\text {an }}\right) .
$$

The functor, restricted to the full subcategory of AMSG consisting of all connected objects, is faithful.

Proof. We will prove this in terms of Harish-Chandra pairs, depending on the established category equivalences in algebraic and analytic situations.

Let $\mathrm{F}$ be an affine algebraic group scheme. Then there is a closed embedding of $\mathrm{F}$ into some $\mathrm{GL}_{n}$. Let $N=n^{2}$. The $\mathrm{GL}_{n}$ is an algebraic matrix group (see [26, Section 4.5, Corollary]), and the group $\mathrm{GL}_{n}(\mathbb{k})$, naturally regarded as a Zariski closed subset of $\mathbb{k}^{N+1}$, includes $\mathrm{F}(\mathbb{k})$ as a Zariski closed subgroup. On the other hand, $\mathrm{GL}_{n}(\mathbb{k})$, regarded as an open sub-manifold of $\mathbb{k}^{N}$, is a Lie group, and it includes $\mathrm{F}(\mathbb{k})$ as a closed topological subgroup with respect to the Euclidian topology; it is finer than the Zariski topology.

Assume that $\mathrm{F}$ is an algebraic matrix group, so that $O_{\mathrm{F}}$ is smooth, and $O_{\mathrm{F}}=\operatorname{Poly}(\mathrm{F}(\mathbb{k}), \mathbb{k})$. Let $d=\operatorname{Kdim}\left(O_{\mathrm{F}}\right)$. The argument of [20, Chapter II, Section 2.3] shows that every point of $F(\mathbb{k})$ has an open (in the Euclidian topology) neighborhood $U \subset \mathbb{k}^{N}$, and polynomials $f_{1}, \ldots, f_{N-d}$ in $\mathbb{k}\left[\mathbf{T}_{1}, \ldots, \mathbf{T}_{N}\right]$, such that $\left(f_{1}, \ldots, f_{N-d}, \mathbf{T}_{1}, \ldots, \mathbf{T}_{d}\right): U \rightarrow \mathbb{k}^{N}$ gives a homeomorphism onto an open set of $\mathbb{k}^{N}$, and $\mathrm{F}(\mathbb{k}) \cap U=\left\{x \in U \mid f_{1}(x)=\cdots=\right.$ $\left.f_{N-d}(x)=0\right\}$. It follows from Item 2) in [18, Part II, Chapter IV, Section 2] that $F(\mathbb{k})$ is a Lie group, which we will denote by $F^{\text {an }}$. Being independent of choice of the embedding $\mathrm{F} \subset \mathrm{GL}_{n}$, the topology on $\left|\mathrm{F}^{\mathrm{an}}\right|$ is defined so that the subsets

$$
B\left(p_{1}, \ldots, p_{r} ; \epsilon\right)=\left\{g \in \mathrm{F}(\mathbb{k})|| g\left(p_{1}\right)|<\epsilon, \ldots,| g\left(p_{r}\right) \mid<\epsilon\right\},
$$

where $\epsilon>0$, and $p_{1}, \ldots, p_{r}$ are finitely many elements of $O_{\mathrm{F}}$, give an open base; see [20. Chapter VII, Section 1.1]. The analytic structure of $F^{\text {an }}$ is precisely what is induced through those local homeomorphisms $\left(q_{1}, \ldots, q_{d}\right)$ : $U \rightarrow \mathbb{k}^{d}$ onto open sets of $\mathbb{k}^{d}$ which are given by polynomial functions $q_{1}, \ldots, q_{d}$ in $O_{\mathrm{F}}$.

We regard $F(\mathbb{k})$ naturally as the ringed subspace of the affine scheme $\operatorname{Spec}\left(O_{\mathrm{F}}\right) ; \mathrm{F}(\mathbb{k})$ thus consists of the maximal ideals of codimension 1, and its structure sheaf is the inverse image of the one $\mathcal{O}_{\mathrm{Spec}\left(O_{\mathrm{F}}\right)}$ of $\operatorname{Spec}\left(O_{\mathrm{F}}\right)$. Recall that $F^{\text {an }}$ is a ringed space, equipped with the sheaf $\mathcal{F}_{F^{a n}}$ of analytic functions. One sees that the identity map

$$
i=\mathrm{id}: \mathrm{F}^{\mathrm{an}} \rightarrow \mathrm{F}(\mathbb{k})
$$


is a morphism of ringed spaces such that $i_{g}^{*}: \mathcal{O}_{\mathrm{F}(\mathbb{k}), g} \rightarrow \mathcal{F}_{\mathrm{Fan}_{, g}}$ turns, by completion, into an isomorphism at every $g \in \mathrm{F}(\mathbb{k})\left(=\left|\mathrm{F}^{\text {an }}\right|\right)$. The isomorphism $\hat{i_{e}^{*}}$ at $e$, which is seen to preserve the $\mathrm{F}(\mathbb{k})$-actions induced by the right adjoint action of the group, gives the identifications

$$
\operatorname{hy}(F)=\operatorname{hy}\left(F^{\text {an }}\right), \quad \operatorname{Lie}(F)=\operatorname{Lie}\left(F^{\text {an }}\right)
$$

of $F(\mathbb{k})$-objects.

Giving a linear representation $\mathrm{F} \rightarrow \mathrm{GL}_{r}$ is the same as giving a group homomorphism $\mathrm{F}(\mathbb{k}) \rightarrow \mathrm{GL}_{r}(\mathbb{k})$ whose composites with the projections $\mathrm{pr}_{i j}$ : $\mathrm{GL}_{r}(\mathbb{k}) \rightarrow \mathbb{k}, 1 \leq i, j \leq r$, (see Section 5.2 ) are polynomial functions; the group homomorphism is, therefore, regarded as an analytic representation $\mathrm{F}^{\text {an }} \rightarrow \mathrm{GL}_{r}(\mathbb{k})$. In view of (6.20), as well, it follows that given an algebraic Harish-Chandra pair $(F, V)$ with $F$ an algebraic matrix group, the pair $\left(F^{a n}, V\right)$, equipped with the same [, ] and the induced analytic module structure, is an (analytic) Harish-Chandra pair as defined by Definition 6.1, Moreover, the assignment $(\mathrm{F}, \mathrm{V}) \mapsto\left(\mathrm{F}^{\mathrm{an}}, \mathrm{V}\right)$ is functorial, and gives rise to the desired functor AMSG $\rightarrow$ SLG.

By [10, Theorem 10] every hyper-super-algebra uniquely arises from a dual Harish-Chandra pair [10, Definiton 6] in a natural manner that dualizes the construction of super-groups from Harish-Chandra pairs, both in algebraic and analytic situations. One sees easily that $(F, V)$ and $\left(F^{a n}, V\right)$ are dualized to the same dual Harish-Chandra pair, $(h y(F), V)=\left(h y\left(F^{a n}\right), V\right)$, from which, therefore, the same hyper-super-algebra, hy $(G)=$ hy $\left(G^{\text {an }}\right)$, arises. Proved is the first equality of (6.19), which, with $P()$ applied, yields the second. The last assertion on faithfulness follows from the first equality, since it is known the functor hy defined on ASG is faithful, when restricted to the full subcategory consisting of all connected ones; see [11, Proposition 21].

One sometimes encounters with such presentations of real or complex super Lie groups that look presenting by use of matrices, algebraic matrix super-groups. Corollary 6.15] justifies such presentations; they can be understood to indicate the super Lie groups which arise from the actually presented algebraic matrix super-groups. (In the real case one has to verify that the associated affine algebraic group scheme is indeed an algebraic matrix group.) Here is a familiar example.

Example 6.16. Suppose that $\mathbb{k}$ is an infinite field of characteristic $\neq 2$. Let $\mathrm{M}=\mathrm{M}_{0} \oplus \mathrm{M}_{1}$ be a finite-dimensional super-vector space with $m=\operatorname{dim}\left(\mathrm{M}_{0}\right)$, $n=\operatorname{dim}\left(\mathrm{M}_{1}\right)$. Let $\mathrm{G}=\mathrm{GL}(\mathrm{M})$ denote the functor which associates to each super-algebra $R$ the group $\mathrm{GL}_{R}(\mathrm{M} \otimes R)$ of all $R$-super-module automorphisms of $\mathrm{M} \otimes R$. In fact this is a smooth affine algebraic super-group scheme; it is represented by the finitely generated Hopf super-algebra

$$
O_{\mathrm{G}}=\mathbb{k}\left[\mathrm{X}_{i j}, \mathrm{Y}_{k \ell}, \operatorname{det}(\mathbf{X})^{-1}, \operatorname{det}(\mathbf{Y})^{-1}\right] \otimes \wedge\left(\mathrm{P}_{i \ell}, \mathrm{Q}_{k j}\right),
$$

where $\mathbf{X}_{i j}, \mathbf{Y}_{k \ell}$ (resp., $\mathbf{P}_{i \ell}, \mathbf{Q}_{k j}$ ) are even (resp., odd) variables, and are presented as entries of the matrix

$$
\left(\begin{array}{ll}
\mathbf{X} & \mathbf{P} \\
\mathbf{Q} & \mathbf{Y}
\end{array}\right)=\left(\begin{array}{cc}
\mathrm{X}_{i j} & \mathrm{P}_{i \ell} \\
\mathrm{Q}_{k j} & \mathrm{Y}_{k \ell}
\end{array}\right), \quad 1 \leq i, j \leq m, 1 \leq k, \ell \leq n
$$


The structure maps of $O_{\mathrm{G}}$ are given by

$$
\begin{aligned}
\Delta\left(\begin{array}{ll}
\mathbf{X} & \mathbf{P} \\
\mathbf{Q} & \mathbf{Y}
\end{array}\right) & =\left(\begin{array}{cc}
\mathbf{X} & \mathbf{P} \\
\mathbf{Q} & \mathbf{Y}
\end{array}\right) \otimes\left(\begin{array}{cc}
\mathbf{X} & \mathbf{P} \\
\mathbf{Q} & \mathbf{Y}
\end{array}\right), \quad \varepsilon\left(\begin{array}{cc}
\mathbf{X} & \mathbf{P} \\
\mathbf{Q} & \mathbf{Y}
\end{array}\right)=\left(\begin{array}{ll}
I & O \\
O & I
\end{array}\right), \\
S\left(\begin{array}{ll}
\mathbf{X} & \mathbf{P} \\
\mathbf{Q} & \mathbf{Y}
\end{array}\right) & =\left(\begin{array}{cc}
\left(\mathbf{X}-\mathbf{P} \mathbf{Y}^{-1} \mathbf{Q}\right)^{-1} & -\mathbf{X}^{-1} \mathbf{P} S(\mathbf{Y}) \\
-\mathbf{Y}^{-1} \mathbf{Q} S(\mathbf{X}) & \left(\mathbf{Y}-\mathbf{Q X} \mathbf{X}^{-1} \mathbf{P}\right)^{-1}
\end{array}\right) .
\end{aligned}
$$

The corresponding algebraic Harish-Chandra pair consists of the affine algebraic group scheme

$$
\mathrm{F}=\mathrm{GL}\left(\mathrm{M}_{0}\right) \times \mathrm{GL}\left(\mathrm{M}_{1}\right),
$$

which is indeed an algebraic matrix group, and the super-vector space

$$
\mathrm{V}=\operatorname{Hom}\left(\mathrm{M}_{0}, \mathrm{M}_{1}\right) \oplus \operatorname{Hom}\left(\mathrm{M}_{1}, \mathrm{M}_{0}\right)(\subset \operatorname{End}(\mathrm{M}))
$$

consisting of the parity-reversing linear endomorphisms of $\mathrm{M}$, on which $\mathrm{F}$ acts from the right so that

$$
v^{g}=g^{-1} \circ v \circ g \text { in } \operatorname{End}_{R}(\mathrm{M} \otimes R),
$$

where $v \in \mathrm{V}, g \in \mathrm{F}(R)$, and $R$ is an arbitrary algebra. The associated $[]:, \mathrm{V} \otimes \mathrm{V} \rightarrow \operatorname{Lie}(\mathrm{F})=\operatorname{End}\left(\mathrm{M}_{0}\right) \oplus \operatorname{End}\left(\mathrm{M}_{1}\right)$ is given by the anti-commutator $[v, w]=v \circ w+w \circ v$. Note that the right $\mathrm{F}$-adjoint action on Lie $(\mathrm{F})$ is given by the same formula as (6.21).

Return to the situation that $\mathbb{k}$ is a complete field. By Corollary 6.15 we have a super Lie group, $G^{\text {an }}$, which we denote by the same symbol GL(M). The corresponding Harish-Chandra pair is $\left(\mathrm{F}^{\mathrm{an}}, \mathrm{V}\right)$ with which is associated the same [,] as above. We remark that $F^{a n}=F(\mathbb{k})$ is the product of the general linear groups of $\mathrm{M}_{0}$ and of $\mathrm{M}_{1}$, which acts on $\mathrm{V}$ and $\operatorname{Lie}\left(\mathrm{F}^{\mathrm{an}}\right)(=$ Lie $(\mathrm{F}))$ by the same formula as (6.21) in the restricted situation $R=\mathbb{k}$.

Remark 6.17. Suppose $\mathbb{k}=\mathbb{C}$. Corollary 6.15 then follows alternatively by applying the following unpublished result due to A. Zubkov and the second-named author, whose proof will be published somewhere else; it is highly expected that an analogous result holds over any complete field of characteristic $\neq 2$. There is a natural functor from the category of smooth locally algebraic super-schemes over $\mathbb{C}$ to the category of complex supermanifolds. It preserves finite products and, therefore, group objects.

6.5. The universal algebraic hull of a super Lie group. Let $G$ be a super Lie group. Given a finite-dimensional super-vector space M, a representation of $G$ on $\mathrm{M}$ is a homomorphism $G \rightarrow \mathrm{GL}(\mathrm{M})$ of super Lie groups. Given such a representation, $\mathrm{M}$ is called a finite-dimensional (left) $G$-supermodule. A (left) G-super-module is a filtered union of finite-dimensional (left) $G$-super-modules.

Let $(F, \mathrm{~V})=\Phi(G)$ be the Harish-Chandra pair corresponding to $G$. Let $\mathcal{B}$ be the associated Hopf super-algebra constructed by Proposition 6.6, and let

$$
G^{\mathrm{al}}=\operatorname{SSp}(\mathcal{B})
$$

denote the affine super-group scheme represented by $\mathcal{B}$. A $G^{\text {al }}$-super-module is naturally identified with a right $\mathcal{B}$-super-comodule. 
Theorem 6.18. Let $\mathrm{M}$ be a super-vector space of possibly infinite dimension. There exist natural one-to-one correspondences among the following three sets:

(a) the set of all $G$-super-module structures on $\mathrm{M}$;

(b) the set of all $G^{\text {al }}$-super-module structures on $\mathrm{M}$;

(c) the set of all pairs $(\cdot, \nabla)$ of an $F$-super-module structure $F \times \mathrm{M} \rightarrow$ $\mathrm{M},(g, m) \mapsto g \cdot m$ on $\mathrm{M}$ and a super-linear map $\mathrm{V} \otimes \mathrm{M} \rightarrow \mathrm{M}$, $v \otimes m \mapsto v \triangleright m$, with $\vee$ supposed to be purely odd, such that

$$
\begin{aligned}
& g \cdot\left(v^{g} \triangleright m\right)=v \triangleright(g \cdot m), \\
& v \triangleright(w \triangleright m)+w \triangleright(v \triangleright m)=[v, w] \triangleright m,
\end{aligned}
$$

where $g \in F, v, w \in \mathrm{V}$ and $m \in \mathrm{M}$. The last $\triangleright$ indicates the action by $\operatorname{Lie}(F)$ induced from the action . by $F$; cf. (5.13).

Proof. The first (and main) step is to prove the one-to-one correspondence (b) $\leftrightarrow$ (c). Recall from Section 6.2 (see Remark 6.8, in particular) that $\mathcal{B}$ is constructed as a quotient of the complete Hopf super-algebra in the wider sense $\hat{\mathcal{A}}$, which is regarded as a complete Hopf super-subalgebra of $(F \ltimes \mathfrak{T}(\mathrm{V}))^{*}$.

Suppose that we are given such a pair $(\cdot, \bullet)$ as in (c) that satisfies (6.22), but may not satisfy $(6.23)$. Then $\bullet$ uniquely extends to a left $\mathfrak{T}(\mathrm{V})$-supermodule structure on $\mathrm{M}$; it will be denoted by the same symbol $\bullet$. Moreover, (6.22) ensures that the extended structure and · make M into a left $F \ltimes \mathfrak{T}(\mathrm{V})$ super-module. Conversely, every left $F \ltimes \mathfrak{T}(\mathrm{V})$-super-module structure on $\mathrm{M}$ uniquely arises in this way, provided the following condition $(\mathrm{An})$ is satisfied: (An) the structure restricted to $F \times \mathrm{M} \rightarrow \mathrm{M}$ is analytic.

Claim. The pair $(\cdot, \nabla)$ above gives rise to a topological right $\hat{\mathcal{A}}$-super-comodule structure on the discrete super-vector space $\mathrm{M}$

$$
\rho: \mathrm{M} \rightarrow \mathrm{M} \hat{\otimes} \hat{\mathcal{A}}=\operatorname{Hom}(\mathfrak{T}(\mathrm{V}), \mathrm{M} \otimes \mathcal{R}(F))
$$

so that

$$
\rho(m)(z)=\rho_{0}(z \bullet m), \quad m \in \mathbf{M}, z \in \mathfrak{T}(\mathrm{V}),
$$

where $\rho_{0}$ denotes the $\mathcal{R}(F)$-super-comodule structure corresponding to the $F$ (or $F^{\mathrm{al}_{-}}$)super-module structure - of the pair. Conversely, every topological right $\hat{\mathcal{A}}$-super-comodule structure on $\mathrm{M}$ uniquely arises in this way.

Indeed, the $F \ltimes \mathfrak{T}(\mathrm{V})$-super-module structure on $\mathrm{M}$ which arises from the pair $(\cdot, \bullet)$ corresponds to the topological right $(F \ltimes \mathfrak{T}(\mathrm{V}))^{*}$-super-comodule structure

$$
\tilde{\rho}: \mathrm{M} \rightarrow \mathrm{M} \hat{\otimes}(F \ltimes \mathfrak{T}(\mathrm{V}))^{*}=\operatorname{Hom}(\mathfrak{T}(\mathrm{V}), \operatorname{Map}(F, \mathrm{M}))
$$

given by

$$
\tilde{\rho}(m)(z)(g)=g \cdot(z \triangleright m), \quad m \in \mathrm{M}, z \in \mathfrak{T}(\mathrm{V}), g \in F ;
$$

see Proposition 2.3. Here $\operatorname{Map}(F, M)$ denotes the super-vector space of all maps $F \rightarrow \mathrm{M}$; it naturally includes $\mathrm{M} \otimes \mathcal{R}(F)$. One sees that $\tilde{\rho}$ takes values in $\mathrm{M} \hat{\otimes} \hat{\mathcal{A}}=\operatorname{Hom}(\mathfrak{T}(\mathrm{V}), \mathrm{M} \otimes \mathcal{R}(F))$; indeed, this is equivalent to the condition (An) above. This proves the Claim. 
It remains to show that the $\rho$ arising from the pair $(\cdot, \bullet)$ takes values in

$$
\mathrm{M} \otimes \mathcal{B}=\operatorname{Hom}_{D}(C, \mathrm{M} \otimes \mathcal{R}(F)) \text { in } \mathrm{M} \hat{\otimes} \hat{\mathcal{A}}=\operatorname{Hom}_{D}(\mathcal{C}, \mathrm{M} \otimes \mathcal{R}(F)),
$$

if and only if the pair satisfies (6.23), where $\mathrm{M} \otimes \mathcal{R}(F)$ is regarded as a left $D$-module, with $D$ acting on the factor $\mathcal{R}(F)$. This is easily seen from $\rho_{0}(a \triangleright m)=a \triangleright \rho_{0}(m)$, where $a \in \operatorname{Lie}(F), m \in \mathrm{M}$. This completes the first step.

It results that given a pair of (c), $M$ is a filtered union of finite-dimensional super-vector subspaces which are stable under the pair of actions. Therefore, to prove $(\mathrm{a}) \leftrightarrow(\mathrm{c})$, we may suppose $\operatorname{dim} \mathrm{M}<\infty$. Then (c) is precisely the set of all morphism in HCP, from $(F, \mathrm{~V})$ to the Harish-Chandra pair of GL(M) given in Example6.16. Hence the desired result follows by Theorem 6.3.

In view of Theorem 6.18, $\mathcal{B}$ and $G^{\text {al }}$ may be called the Hopf super-algebra of analytic representative functions on the super Lie group $G$, and the universal algebraic hull of $G$, respectively. These are super-analogues of the corresponding objects investigated by Hochschild and Mostow [6] one of them is the universal algebraic hull $F^{\text {al }}$ of a Lie group $F$, discussed before.

\section{New Construction of the Quotient $G / H$}

7.1. Super Lie subgroups. Let $G$ be a super Lie group. By a super Lie subgroup of $G$, we mean a super Lie group $H$, given a morphism $\phi: H \rightarrow$ $G$ of super Lie groups such that the associated morphism $\left(\phi_{\text {red }}, \operatorname{Lie}(\phi)_{1}\right)$ : $\left(H_{\text {red }}, \operatorname{Lie}(H)_{1}\right) \rightarrow\left(G_{\text {red }}, \operatorname{Lie}(G)_{1}\right)$ of Harish-Chandra pairs satisfies

(i) $\phi_{\text {red }}: H_{\text {red }} \rightarrow G_{\text {red }}$ is a closed embedding, and

(ii) $\operatorname{Lie}(\phi)_{1}\left(=\left.\operatorname{Lie}(\phi)\right|_{\operatorname{Lie}(H)_{1}}\right): \operatorname{Lie}(H)_{1} \rightarrow \operatorname{Lie}(G)_{1}$ is injective.

Obviously, (ii) may be replaced with one of the following conditions which are equivalent to each other:

(iii) $\operatorname{Lie}(\phi): \operatorname{Lie}(H) \rightarrow \operatorname{Lie}(G)$ is injective;

(iii) $)^{\prime} \operatorname{hy}(\phi): \operatorname{hy}(H) \rightarrow \operatorname{hy}(G)$ is injective.

We present $\phi$ so as $H \subset G$, and regard $\phi_{\text {red }}$, $\operatorname{Lie}(\phi)$ and hy $(\phi)$ as inclusions. The Lie group $H_{\text {red }}$ is, therefore, a Lie subgroup of $G_{\text {red }}$; see [27, Definition 6.6, Proposition 6.7].

Notice that $G_{\text {red }}$ is a super Lie subgroup of $G$. One sees easily that the following are equivalent:

(a) The inclusion $G_{\text {red }} \hookrightarrow G$ splits as a morphism of super Lie groups;

(b) The Lie super-algebra $\operatorname{Lie}(G)$ of $G$ is $\mathbb{N}$-graded, or equivalently, $\left[\operatorname{Lie}(G)_{1}, \operatorname{Lie}(G)_{1}\right]=0$.

If these are satisfied we say that $G$ is $\mathbb{N}$-graded. We remark that $G_{\text {red }} \hookrightarrow G$ then has a canonical retraction since the corresponding morphism $\left(G_{\text {red }}, 0\right) \rightarrow$ $\left(G_{\text {red }}, \operatorname{Lie}(G)_{1}\right)$ of Harish-Chandra pairs does.

Modify the Harish-Chandra pair $\left(G_{\mathrm{red}}\right.$, $\left.\operatorname{Lie}(G)_{1}\right)$ corresponding to $G$, replacing the equipped bracket with the zero map. To the modified HarishChandra pair corresponds an $\mathbb{N}$-graded super Lie group, which we denote by $\operatorname{gr} G$. This $\operatorname{gr} G$ is intrinsically constructed from the original $G$, by applying gr to the structure sheaf of $G$ and to the sheaf-morphisms $\Delta, S$, such as given by (5.1), that are associated with the product $\mu$ and the inverse $\iota$ on $G$; see [25, Section 4], for example. 
7.2. The theorem on $G / H$. Let $G=(|G|, \mathcal{O})$ be a super Lie group with the associated Lie group $G_{\text {red }}=(|G|, \mathcal{F})$. We thus denote the structure sheaves $\mathcal{O}_{G}, \mathcal{F}_{G_{\text {red }}}$ by $\mathcal{O}, \mathcal{F}$, simply. We set $\mathrm{W}=\left(\operatorname{Lie}(G)_{1}\right)^{*}$.

Recall that $G$ is a group-object in SMFD. A left (resp., right) $G$-equivariant object will be called a left (resp., right) $G$-equivariant super-manifold. Note that $G$ itself is left and right $G$ - and $G_{\text {red-equivariant. Recall from Propo- }}$ sition 6.11 and (6.4) the canonical sheaf-isomorphism $\eta$ composed with a non-canonical one,

$$
\mathcal{O} \stackrel{\simeq}{\longrightarrow} \mathcal{F} \otimes \wedge(\mathrm{W})
$$

In view of (6.9) (suppose $a=1$ there), this last gives an isomorphism

$$
G_{\text {red }} \times(\{*\}, \wedge(\mathrm{W})) \stackrel{\simeq}{\longrightarrow} G
$$

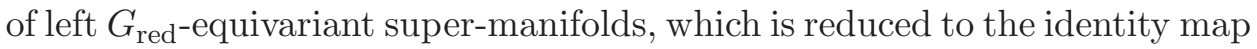
$\operatorname{id}_{G_{\text {red }}}$ on $G_{\text {red }}$. We have an analogous, right $G_{\text {red-equivariant isomorphism }}$

$$
(\{*\}, \wedge(\mathrm{W})) \times G_{\mathrm{red}} \stackrel{\simeq}{\longrightarrow} G,
$$

which will be of use, in fact. If $G$ is $\mathbb{N}$-graded, we can choose as the last two isomorphisms, canonical isomorphisms of super Lie groups, replacing the direct products on the left-hand side with semi-direct products; see the remark given in the second last paragraph of the preceding subsection.

Recall that $H_{\text {red }}$ is a Lie subgroup of $G_{\text {red }}$. Notice from Proposition 5.17 that $H_{\text {red }}$ acts analytically by left adjoint on the hyper-super-algebra hy $(H)$ of $H$, so that we have the super-cocommutative Hopf super-algebra of semidirect product

$$
J:=\operatorname{hy}(H) \rtimes H_{\text {red }},
$$

in which every element of $H_{\text {red }}$ is supposed to be group-like. This includes

$$
K:=\operatorname{hy}\left(H_{\text {red }}\right) \rtimes H_{\text {red }}
$$

as the largest purely even Hopf super-subalgebra. Just as above and in what follows, as well, (super-)modules over a Lie group or its actions are explicitly said to be analytic, if that is the case; cf. Convention 5.10 .

It is known (see [18, Theorem 1 on Page 108]) that the set of left cosets

$$
Q:=G_{\text {red }} / H_{\text {red }},
$$

equipped with the quotient topology, is uniquely made into a manifold so that the quotient morphism which we denote by

$$
\pi: G_{\text {red }} \rightarrow Q=G_{\text {red }} / H_{\text {red }}
$$

is a submersion, that is, $T_{g} \pi: T_{g}\left(G_{\text {red }}\right) \rightarrow T_{\pi(g)}(Q)$ is surjective for every $g \in$ $G_{r e d}$; the symbols are and will be used to present the underlying topological spaces $\left|G_{\text {red }}\right|,\left|H_{\text {red }}\right|,|Q|$ or the continuous map $|\pi|$, as well. Given an open set $U$ of $Q$, the open submanifold $\pi^{-1}(U)$ of $G_{\text {red }}$ is right $H_{\text {red-stable }}$ (i.e., stable under the right multiplication by $H_{\text {red }}$ ), and so the induced action makes $\mathcal{F}\left(\pi^{-1}(U)\right)$ into a left $H_{\text {red-module algebra. With this action }}$ combined with the natural action by hy $\left(H_{\text {red }}\right)$ on $\mathcal{F}$ (see Proposition 5.14), $\mathcal{F}\left(\pi^{-1}(U)\right)$ turns into a left $K$-module algebras. Moreover, $\pi_{*} \mathcal{F}$ turns into a sheaf of those algebras; see the proof of Lemma 7.1] below. One sees that the structure sheaf $\mathcal{F}_{Q}$ of $Q$ associates to every open set $U \subset Q$ the subalgebra

$$
\mathcal{F}_{Q}(U)=\mathcal{F}\left(\pi^{-1}(U)\right)^{K}
$$


of $\mathcal{F}\left(\pi^{-1}(U)\right)$ which consists of the $K$-invariants; in general, an invariant is defined to be an element $f$ such that $a \triangleright f=\epsilon(a) f$ for all elements $a$ of the Hopf (super-)algebra in question. It is easy to see $\mathcal{F}_{Q}(U) \subset \mathcal{F}\left(\pi^{-1}(U)\right)^{K}$, and the equality follows by passing to the stalks, using the fact: $\pi$ is a

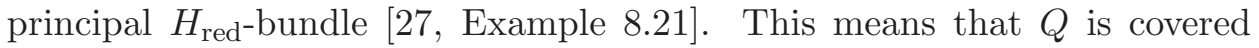
by admissible open subsets. Here we say that an open set $\emptyset \neq U \subset Q$ is admissible if there exists a trivialization

$$
\operatorname{triv}_{U}: U \times H_{\text {red }} \stackrel{\simeq}{\longrightarrow} \pi^{-1}(U),
$$

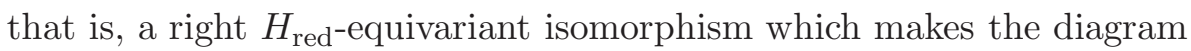

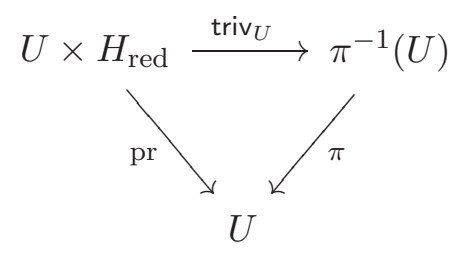

commutative, where pr denotes the natural projection.

Notice from Proposition 5.14 that $\mathcal{O}$ is a sheaf of left hy $(H)$-super-module super-algebras.

Lemma 7.1. Given an open set $U$ of $Q, \pi^{-1}(U)$, regarded as an open super-submanifold of $G$, is right $H_{\mathrm{red}}$-stable. The induced left $H_{\mathrm{red}}$-action on $\mathcal{O}\left(\pi^{-1}(U)\right)$, combined with the action by hy $(H)$ noticed above, makes $\mathcal{O}\left(\pi^{-1}(U)\right)$ into a left $J$-super-module super-algebra. This action by $J$ is natural in $U$.

Proof. The first stability follows from (7.1), while the last naturality follows since the actions by $H_{\text {red }}$ and by hy $(H)$ are both natural.

To complete the proof, it suffices to prove that for $h \in H_{\text {red }}, a \in \operatorname{hy}(H)$, the induced actions

$$
h_{a} \text { and } h \circ a \circ h^{-1}
$$

on completed stalks $\hat{\mathcal{O}}_{g}, g \in \pi^{-1}(U)$, coincide. Notice that the action by $h$ is

$$
\hat{\mathcal{O}}_{g h} \stackrel{\hat{\Delta}_{g, h}}{\longrightarrow} \hat{\mathcal{O}}_{g} \hat{\otimes} \hat{\mathcal{O}}_{h} \stackrel{\text { id } \hat{\otimes} \hat{\epsilon}_{h}}{\longrightarrow} \hat{\mathcal{O}}_{g} \text {. }
$$

Then the desired result follows easily from the relation (6.16) modified for the opposite-sided actions.

Definition 7.2. We let $G / H$ denote the super-ringed space with the underlying topological space $Q=G_{\text {red }} / H_{\text {red }}$, equipped with the sheaf which associates to every open set $U$,

$$
\mathcal{O}_{G / H}(U):=\mathcal{O}\left(\pi^{-1}(U)\right)^{J},
$$

the super-subalgebra of $J$-invariants in $\mathcal{O}\left(\pi^{-1}(U)\right)$; this is seen to define a sheaf by the naturality shown in the preceding lemma.

We define a morphism of super-ringed spaces

$$
\Pi=\left(\pi, \Pi^{*}\right): G \rightarrow G / H,
$$

which consists of the continuous map $\pi:|G|=G_{\text {red }} \rightarrow Q=|G / H|$ and the the sheaf-morphism

$$
\Pi^{*}: \mathcal{O}_{G / H}(U)=\mathcal{O}\left(\pi^{-1}(U)\right)^{J} \hookrightarrow \mathcal{O}\left(\pi^{-1}(U)\right)=\pi_{*} \mathcal{O}(U)
$$


given by the inclusion.

Theorem 7.3. We have the following.

(1) The super-ringed space $G / H$ is a super-manifold, whence $\Pi: G \rightarrow$ $G / H$ is a morphism in the category SMFD of super-manifolds. This $\Pi$ has the following properties:

(i) For every $g \in|G|, d \Pi_{g}: T_{g} G \rightarrow T_{\pi(g)}(G / H)$ is surjective;

(ii) $G \times H \rightrightarrows G \stackrel{\Pi}{\longrightarrow} G / H$ is a co-equalizer diagram in SMFD, where the paired arrows indicate the product on $G$ restricted to $G \times H$ and the natural projection.

(2) Given an admissible open set $U$ of $Q$ and a trivialization such as in (7.3), $\pi^{-1}(U)$, regarded as an open super-submanifold of $G$, is right $H$-stable, and there exists an isomorphism

$$
\operatorname{Triv}_{U}:\left(U,\left.\mathcal{O}_{G / H}\right|_{U}\right) \times H \stackrel{\simeq}{\longrightarrow}\left(\pi^{-1}(U),\left.\mathcal{O}\right|_{\pi^{-1}(U)}\right)
$$

of right $H$-equivariant super-manifolds such that

$$
\left(\operatorname{Triv}_{U}\right)_{\mathrm{red}}=\operatorname{triv}_{U}
$$

and the diagram

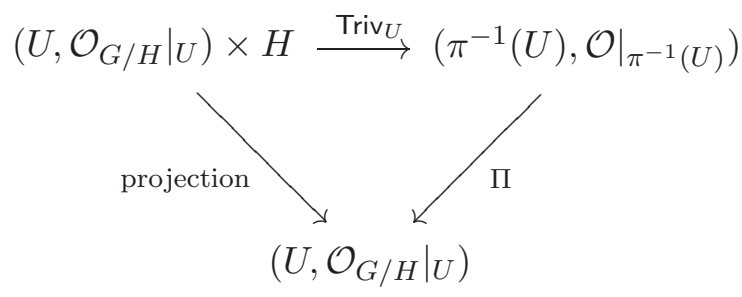

is commutative.

Remark 7.4. (1) Due to the property (i) shown in Pat 1 above, the supermanifold $G / H$ may be called the quotient of $G$ by $H$. Due to the property shown in Part 2, $\Pi: G \rightarrow G / H$ may be called a principal super $H$-bundle. In fact, there is more shown: any non-super trivialization can lift to one in the super context. This result is likely new even when $\mathbb{k}=\mathbb{R}$ or $\mathbb{C}$.

(2) Very recently, the second- and third-named authors [15] applied Hopfalgebraic techniques to give a new construction of the quotient $G / H$ in the algebraic situation where $G$ is an affine algebraic super-group scheme over an arbitrary field of characteristic $\neq 2$, and $H$ is a closed super-subgroup scheme. Pursuing an analogue of the construction in the present analytic situation resulted successfully in the theorem above; it was a surprise to the authors.

7.3. Proof of the theorem. Choose arbitrarily an admissible open set $U \subset Q$ and a trivialization such as in (7.3), and fix them. One sees that the direct image $\operatorname{pr}_{*} \mathcal{F}_{U \times H_{\text {red }}}$ of the structure sheaf $\mathcal{F}_{U \times H_{\text {red }}}$ of $U \times H_{\text {red }}$ is a sheaf of left $K$-module algebras, and the sheaf-isomorphism

$$
\left(\operatorname{triv}_{U}\right)^{*}:\left.\pi_{*} \mathcal{F}\right|_{U} \stackrel{\simeq}{\longrightarrow} \operatorname{pr}_{*} \mathcal{F}_{U \times H_{\text {red }}}
$$

associated with $\operatorname{triv}_{U}$ preserves the $K$-action. Compose the inverse $\left(\left(\operatorname{triv}_{U}\right)^{*}\right)^{-1}$ with the algebra map $\mathcal{F}_{H_{\text {red }}}\left(H_{\text {red }}\right) \rightarrow \mathcal{F}_{U \times H_{\text {red }}}\left(U \times H_{\text {red }}\right)$ associated with the projection $U \times H_{\text {red }} \rightarrow H_{\text {red }}$, and restrict the resulting $\left.\mathcal{F}_{H_{\text {red }}}\left(H_{\text {red }}\right) \rightarrow \pi_{*} \mathcal{F}\right|_{U}$ 
to the Hopf algebra $\mathcal{R}\left(H_{\text {red }}\right)$ of the analytic representative functions on $H_{\text {red }}$; see Section 5.2. We denote the thus (non-canonically) obtained map by

$$
\varrho_{H_{\mathrm{red}}}:\left.\mathcal{R}\left(H_{\mathrm{red}}\right) \rightarrow \pi_{*} \mathcal{F}\right|_{U},
$$

through which $\left.\pi_{*} \mathcal{F}\right|_{U}$ turns into a sheaf of $K$-module algebras over $\mathcal{R}\left(H_{\text {red }}\right)$ (that is, every $\varrho_{H_{\text {red }}, V}: \mathcal{R}\left(H_{\text {red }}\right) \rightarrow \mathcal{F}\left(\pi^{-1}(V)\right)$ is a $K$-module algebra map) on $U$. On the other hand we let

$$
\sigma_{G_{\text {red }}}:\left.\mathcal{R}\left(G_{\text {red }}\right) \rightarrow \pi_{*} \mathcal{F}\right|_{U}
$$

denote the canonical map $\left.\mathcal{F}\left(G_{\text {red }}\right) \rightarrow \mathcal{F}\right|_{U}$ restricted to the Hopf algebra $\mathcal{R}\left(G_{\text {red }}\right)$ of the analytic representative functions on $G_{\text {red }}$, through which $\left.\pi_{*} \mathcal{F}\right|_{U}$ turns into a sheaf of $K$-module algebras over $\mathcal{R}\left(G_{\text {red }}\right)$ on $U$.

Since $\operatorname{Lie}(G)_{1}$ is an analytic right $G_{\text {red }}$-module by adjoint (see Remark 5.18), $\mathrm{W}=\left(\operatorname{Lie}(G)_{1}\right)^{*}$ is an analytic left module over $G_{\text {red }}$ and hence over $H_{\text {red }}$. Let $\mathrm{W}_{H}=\left(\operatorname{Lie}(H)_{1}\right)^{*}$. This as well is an analytic left $H_{\text {red-module. }}$ Recall $\operatorname{Lie}(H) \subset \operatorname{Lie}(G)$, and define an analytic left $H_{\text {red-module by }}$

$$
\mathbf{Z}=\left(\operatorname{Lie}(G)_{1} / \operatorname{Lie}(H)_{1}\right)^{*} .
$$

Alternatively, this is defined by the short exact sequence $0 \rightarrow \mathrm{Z} \rightarrow \mathrm{W} \rightarrow$ $\mathrm{W}_{H} \rightarrow 0$ of analytic left $H_{\text {red-modules. Recall that analytic left } H_{\text {red }^{-}}}$ modules are identified with right $\mathcal{R}\left(H_{\text {red }}\right)$-comodules. Such comodules are naturally regarded as left $K$-modules by virtue of the canonical Hopf paring $K \times \mathcal{R}\left(H_{\text {red }}\right) \rightarrow \mathbb{k} ;$ cf. (5.11)

By a $\left(K,\left.\pi_{*} \mathcal{F}\right|_{U}\right)$-module (sheaf) we mean a $\left.\pi_{*} \mathcal{F}\right|_{U}$-module $\mathcal{M}$ which is at the same time a sheaf of left $K$-modules such that the structure $\left.\pi_{*} \mathcal{F}\right|_{U} \otimes$ $\mathcal{M} \rightarrow \mathcal{M}$ is $K$-linear. A morphism of such modules is a $K$-linear and $\left.\pi_{*} \mathcal{F}\right|_{U^{-}}$ linear one. We are going to define two morphisms of $\left(J,\left.\pi_{*} \mathcal{F}\right|_{U}\right)$-modules,

$$
\left.\left.\left.\mathrm{W} \otimes \pi_{*} \mathcal{F}\right|_{U} \stackrel{\tilde{\kappa}_{\mathrm{W}}^{-1}}{\longrightarrow} \pi_{*} \mathcal{F}\right|_{U} \otimes \mathrm{W} \stackrel{\tilde{\theta}}{\longrightarrow} \pi_{*} \mathcal{F}\right|_{U} \otimes \mathbf{Z},
$$

the first of which is the inverse of an isomorphism $\tilde{\kappa}_{\mathrm{W}}$.

In general, given a right $\mathcal{R}\left(H_{\text {red }}\right)$-comodule $\mathrm{M}$ with the structure $\mathrm{M} \rightarrow$ $\mathrm{M} \otimes \mathcal{R}\left(H_{\text {red }}\right), m \mapsto m^{(0)} \otimes m^{(1)}$, we define a map by

$$
\nu_{\mathrm{M}}: \mathcal{R}\left(H_{\text {red }}\right) \otimes \mathrm{M} \rightarrow \mathrm{M} \otimes \mathcal{R}\left(H_{\text {red }}\right), \nu_{\mathrm{M}}(p \otimes m)=m^{(0)} \otimes p m^{(1)} .
$$

In fact this is an isomorphism of $\mathcal{R}\left(H_{\text {red }}\right)$-Hopf modules [21, p.83]. The inverse is given by

$$
\nu_{\mathrm{M}}^{-1}(m \otimes p)=p S_{\mathcal{R}\left(H_{\mathrm{red}}\right)}\left(m^{(1)}\right) \otimes m^{(0)} .
$$

In the obvious manner, $\mathcal{R}\left(H_{\text {red }}\right) \otimes \mathbf{M}$ and $\mathbf{M} \otimes \mathcal{R}\left(H_{\text {red }}\right)$ both are regarded as $\mathcal{R}\left(H_{\text {red }}\right)$-modules. The former is regarded as the tensor product of two right $\mathcal{R}\left(H_{\text {red }}\right)$-comodules, while the latter is regarded as the right $\mathcal{R}\left(H_{\text {red }}\right)$ comodule $\mathcal{R}\left(H_{\text {red }}\right)$ tensored with the vector space M. Being $\mathcal{R}\left(H_{\text {red }}\right)$-colinear, $\nu_{\mathrm{M}}$ is $K$-linear. Choose arbitrarily a linear retraction

$$
\text { ret }: \mathrm{W} \rightarrow \mathrm{Z}
$$


of the inclusion $\mathrm{Z} \hookrightarrow \mathrm{W}$, and define $\theta$ to be the unique map that makes

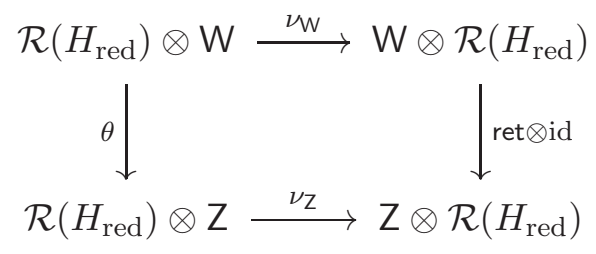

into a commutative diagram of $\mathcal{R}\left(H_{\text {red }}\right)$-Hopf modules; it is easy to express this $\theta$ explicitly, but we will not use the expression. Let

$$
\tilde{\theta}:\left.\left.\pi_{*} \mathcal{F}\right|_{U} \otimes \mathrm{W} \rightarrow \pi_{*} \mathcal{F}\right|_{U} \otimes \mathbf{Z}
$$

be the base extension of $\theta$ along $\varrho_{H_{\text {red }}}$. This $\tilde{\theta}$ is the second one of (7.4), and is seen, indeed, to be a morphism between two $\left(K,\left.\pi_{*} \mathcal{F}\right|_{U}\right)$-modules.

For the $\nu_{\mathrm{W}}$ above, $\mathrm{W}$ is regarded as a right $\mathcal{R}\left(H_{\text {red }}\right)$-comodule. When it is regarded as an original, right $\mathcal{R}\left(G_{\text {red }}\right)$-comodule, an analogous isomorphism

$$
\kappa_{\mathrm{W}}: \mathcal{R}\left(G_{\text {red }}\right) \otimes \mathrm{W} \stackrel{\simeq}{\longrightarrow} \mathrm{W} \otimes \mathcal{R}\left(G_{\text {red }}\right)
$$

of $\mathcal{R}\left(G_{\text {red }}\right)$-Hopf modules is defined in the obvious manner. We let

$$
\tilde{\kappa}_{\mathrm{W}}:\left.\left.\pi_{*} \mathcal{F}\right|_{U} \otimes \mathrm{W} \stackrel{\simeq}{\longrightarrow} \mathrm{W} \otimes \pi_{*} \mathcal{F}\right|_{U}
$$

be its base extension along $\sigma_{G_{\text {red }}}$, which is seen to be a morphism between two $\left(K,\left.\pi_{*} \mathcal{F}\right|_{U}\right)$-modules. Its inverse is the first one of (7.4).

Let

$$
\Theta=\tilde{\theta} \circ \tilde{\kappa}_{\mathrm{W}}^{-1}:\left.\left.\mathrm{W} \otimes \pi_{*} \mathcal{F}\right|_{U} \rightarrow \pi_{*} \mathcal{F}\right|_{U} \otimes \mathrm{Z}
$$

be the composite presented by (7.4).

Lemma 7.5. This $\Theta$ is a retraction of the injection of $\left(K,\left.\pi_{*} \mathcal{F}\right|_{U}\right)$-modules

$$
\left.\tilde{\kappa}_{\mathrm{W}}\right|_{\left.\mathrm{Z} \otimes \pi_{*} \mathcal{F}\right|_{U}}:\left.\left.\mathrm{Z} \otimes \pi_{*} \mathcal{F}\right|_{U} \rightarrow \pi_{*} \mathcal{F}\right|_{U} \otimes \mathrm{W}
$$

that is, the restriction of $\tilde{\kappa}_{\mathrm{W}}$ to $\left.\mathbf{Z} \otimes \pi_{*} \mathcal{F}\right|_{U}$.

Proof. This follows since one sees from the last commutative diagram that $\theta$, restricted to $\left.\mathbf{Z} \otimes \pi_{*} \mathcal{F}\right|_{U}$, turns into the identity.

Remark 7.6. (1) Let

$$
\tilde{\nu}_{\mathrm{W}}:\left.\left.\pi_{*} \mathcal{F}\right|_{U} \otimes \mathrm{W} \rightarrow \mathrm{W} \otimes \pi_{*} \mathcal{F}\right|_{U}, \quad \tilde{\nu}_{\mathrm{Z}}:\left.\left.\pi_{*} \mathcal{F}\right|_{U} \otimes \mathbf{Z} \rightarrow \mathbf{Z} \otimes \pi_{*} \mathcal{F}\right|_{U}
$$

be the base extensions of $\nu_{\mathrm{W}}$ and $\nu_{\mathrm{Z}}$ along $\varrho_{H_{\mathrm{red}}}$. For later use define

$$
\Xi:=\tilde{\nu}_{\mathrm{Z}} \circ \Theta:\left.\left.\mathrm{W} \otimes \pi_{*} \mathcal{F}\right|_{U} \rightarrow \mathrm{Z} \otimes \pi_{*} \mathcal{F}\right|_{U}
$$

We remark that this $\Xi$ does not necessarily equal ret $\otimes \mathrm{id}_{\left.\pi_{*} \mathcal{F}\right|_{U}}$ since $\tilde{\nu}_{\mathrm{W}} \circ$ $\tilde{\kappa}_{\mathrm{W}}^{-1} \neq \mathrm{id}_{\left.\mathrm{W} \otimes \pi_{*} \mathcal{F}\right|_{U}}$, in general.

(2) Our $\tilde{\theta}, \Theta$ are analogues of the $\theta, \theta^{\prime}$ of [15, (4.14), (4.16)], respectively; see Remark 7.4 (2).

Recall that the sheaf $\mathcal{O}$ is hy $(H)$-super-module super-algebras. Given an open set $V$ of $|G|$, the set

$$
\operatorname{Hom}_{\text {hy }\left(H_{\text {red }}\right)}(\text { hy }(H), \mathcal{O}(V))
$$

of all left hy $\left(H_{\text {red }}\right)$-module maps hy $(H) \rightarrow \mathcal{O}(V)$ form a super-algebra with respect to the convolution-product, which is non-canonically isomorphic to 
$\mathcal{O}(V) \otimes \wedge(\mathrm{W})$. This is, moreover, a left hy $(H)$-super-module with respect to the structure induced from the right multiplication by hy $(H)$ on itself. Thus there arises another sheaf of hy $(H)$-super-module super-algebras

$$
\operatorname{Hom}_{\text {hy }\left(H_{\text {red }}\right)}(\text { hy }(H), \mathcal{O})
$$

on $|G|$, which associates $\operatorname{Hom}_{\text {hy }\left(H_{\text {red }}\right)}($ hy $(H), \mathcal{O}(V))$ to every $V$.

We see that

$$
\Lambda_{V}: \mathcal{O}(V) \rightarrow \operatorname{Hom}_{\text {hy }\left(H_{\text {red }}\right)}(\operatorname{hy}(H), \mathcal{O}(V)), \Lambda_{V}(p)(a)=a \triangleright p
$$

defines a hy $(H)$-super-linear sheaf-morphism

$$
\Lambda: \mathcal{O} \rightarrow \operatorname{Hom}_{\text {hy }\left(H_{\text {red }}\right)}(\text { hy }(H), \mathcal{O}) .
$$

Recall that the direct image $\pi_{*} \mathcal{O}$ of $\mathcal{O}$ is a sheaf of $J$-super-module superalgebras. We wish to see that

$$
\pi_{*} \operatorname{Hom}_{\mathrm{hy}\left(H_{\mathrm{red}}\right)}(\mathrm{hy}(H), \mathcal{O})\left(=\operatorname{Hom}_{\mathrm{hy}\left(H_{\mathrm{red}}\right)}\left(\mathrm{hy}(H), \pi_{*} \mathcal{O}\right)\right)
$$

is as well.

Lemma 7.7. This last sheaf on $Q$ turns into a sheaf of $J$-super-module super-algebras by defining the $H_{\mathrm{red}^{-}}$action

$$
{ }^{h} \varphi: \text { hy }(H) \rightarrow \mathcal{O}\left(\pi^{-1}(U)\right), a \mapsto^{h}\left(\varphi\left({ }^{-1} a\right)\right),
$$

where $U \subset Q$ is any open set, $h \in H_{\text {red }}$ and $\varphi \in \operatorname{Hom}_{\mathrm{hy}\left(H_{\mathrm{red}}\right)}(\operatorname{hy}(H)$, $\left.\mathcal{O}\left(\pi^{-1}(U)\right)\right)$. Moreover, the sheaf-morphism

$$
\pi_{*} \Lambda: \pi_{*} \mathcal{O} \rightarrow \pi_{*} \operatorname{Hom}_{\mathrm{hy}\left(H_{\mathrm{red}}\right)}(\mathrm{hy}(H), \mathcal{O}) .
$$

is then J-super-linear.

Proof. This follows since one sees that

$$
{ }^{h}(a \triangleright p)=\left({ }^{h} a\right) \triangleright\left({ }^{h} p\right), \quad{ }^{h}\left(\left({ }^{h^{-1}} a\right) \triangleright p\right)={ }^{h}\left({ }^{-1} a\right) \triangleright\left({ }^{h} p\right)=a \triangleright\left({ }^{h} p\right),
$$

where $h \in H_{\text {red }}, a \in \operatorname{hy}(H)$ and $p \in \mathcal{O}\left(\pi^{-1}(U)\right)$.

Let us return to the situation that $U$ is a fixed, admissible open set of $Q$. Choose an isomorphism such as in (7.1) and let

$$
\mathcal{O} \stackrel{\simeq}{\longrightarrow} \wedge(\mathrm{W}) \otimes \mathcal{F}
$$

be the associated sheaf-isomorphism. We let

$$
\Sigma_{U}:\left.\left.\left.\pi_{*} \mathcal{O}\right|_{U} \stackrel{\simeq}{\longrightarrow} \wedge(\mathrm{W}) \otimes \pi_{*} \mathcal{F}\right|_{U} \stackrel{\wedge_{\left.\pi_{*} \mathcal{F}\right|_{U}}(\Xi)}{\longrightarrow} \wedge(\mathrm{Z}) \otimes \pi_{*} \mathcal{F}\right|_{U},
$$

be its direct image under $\pi$, composed with the sheaf-morphism $\wedge_{\left.\pi_{*} \mathcal{F}\right|_{U}}(\Xi)$ over $\left.\pi_{*} \mathcal{F}\right|_{U}$ which naturally arises from the $\Xi$ in $(7.5)$. The thus obtained morphism is seen to be $K$-linear. Apply $\operatorname{Hom}_{\text {hy }\left(H_{\text {red }}\right)}($ hy $(H)$, $)$ and compose with the $\pi_{*} \Lambda$ restricted to $U$. We let

$$
\Omega_{U}:\left.\pi_{*} \mathcal{O}\right|_{U} \rightarrow \operatorname{Hom}_{\mathrm{hy}\left(H_{\mathrm{red}}\right)}\left(\mathrm{hy}(H),\left.\wedge(\mathrm{Z}) \otimes \pi_{*} \mathcal{F}\right|_{U}\right)
$$

be the resulting sheaf-morphism.

Proposition 7.8. We have the following.

(1) This $\Omega_{U}$ is a J-super-linear isomorphism of sheaves on $U$, such that

$$
\left(\Omega_{U}\right)_{\mathrm{red}}=\mathrm{id}_{\left.\pi_{*} \mathcal{F}\right|_{U}} .
$$


(2) The restriction to the $J$-invariants gives an isomorphism

$$
\left(\Omega_{U}\right)^{J}:\left.\mathcal{O}_{G / H}\right|_{U}=\left.\mathcal{O}\left(\pi^{-1}()\right)^{J} \stackrel{\simeq}{\longrightarrow} \wedge(Z) \otimes \mathcal{F}_{Q}\right|_{U}
$$

of sheaves on $U$. Hence, $G / H$, being locally a s-split super-manifold, is a super-manifold.

Proof. (1) Non-trivial is to prove that $\Omega_{U}$ is an isomorphism. For this it suffices to prove that the analogous sheaf-morphism

$$
\Omega_{U}^{\prime}:\left.\pi_{*} \mathcal{O}\right|_{U} \rightarrow \operatorname{Hom}_{\mathrm{hy}\left(H_{\mathrm{red}}\right)}\left(\mathrm{hy}(H),\left.\pi_{*} \mathcal{F}\right|_{U} \otimes \wedge(\mathrm{Z})\right),
$$

which is constructed as $\Omega_{U}$ but with $\wedge(\Xi)$ replaced by $\wedge(\Theta)$, is isomorphic. We have to prove that $\operatorname{gr}\left(\Omega_{U}^{\prime}\right)$ is isomorphic. But we wish, equivalently, to prove that $\Omega_{U}^{\prime}$ is isomorphic, assuming $G=\operatorname{gr} G$, or that $G$ is $\mathbb{N}$-graded; see Section 7.1. We should then take the isomorphism (7.6) (or (7.1)), which was used to construct $\Omega_{U}^{\prime}$, as the canonical one. The desired result follows by essentially the same argument as proving [15, Proposition 4.8] (see the argument of proving that the $\left(\omega_{\theta}\right)_{P}$ in [15, (4.19)] is isomorphic); it depends on the equivalence from the category $\operatorname{SMod}_{\wedge(\mathrm{W})}^{\wedge\left(\mathrm{W}_{H}\right)}$ of $\left(\wedge\left(\mathrm{W}_{H}\right), \wedge(\mathrm{W})\right)$-Hopf super-modules to the category $\operatorname{SMod}_{\wedge}(\mathrm{Z})$ of $\wedge(\mathrm{Z})$-super-modules (see [15, Theorem $3.1(1)])$, which associates to each $M \in \operatorname{SMod}_{\wedge(\mathrm{W})}^{\wedge\left(\mathrm{W}_{H}\right)}$, the $\wedge(\mathrm{Z})$ super-module $\mathrm{M}^{c o \wedge}\left(\mathrm{W}_{H}\right)=\left\{m \in \mathrm{M} \mid m^{(0)} \otimes m^{(1)}=m \otimes 1\right\}$ of right $\wedge\left(\mathrm{W}_{H}\right)$ coinvariants in $\mathrm{M}$; the equivalence is ensured by the fact that $\mathrm{W} \rightarrow \mathrm{W}_{H}$ is surjective. As in the proof for $\left(\omega_{\theta}\right)_{P}$ cited above, one sees that under the assumption above, $\Omega_{U}^{\prime}$ is a morphism in $\operatorname{SMod}_{\wedge(\mathrm{W})}^{\wedge\left(\mathrm{W}_{H}\right)}$. It will follow by the category-equivalence above that $\Omega_{U}^{\prime}$ is isomorphic, as desired, if one sees that the restriction

$\left(\Omega_{U}^{\prime}\right)^{c o \wedge\left(\mathrm{W}_{H}\right)}:\left(\left.\pi_{*} \mathcal{O}\right|_{U}\right)^{c o \wedge\left(\mathrm{W}_{H}\right)} \rightarrow\left(\operatorname{Hom}_{\mathrm{hy}\left(H_{\mathrm{red}}\right)}\left(\mathrm{hy}(H),\left.\pi_{*} \mathcal{F}\right|_{U} \otimes \wedge(\mathrm{Z})\right)\right)^{\operatorname{co} \wedge\left(\mathrm{W}_{H}\right)}$

to the right $\wedge\left(\mathrm{W}_{H}\right)$-coinvariants is isomorphic. The domain is naturally identified with $\left.\pi_{*} \mathcal{F}\right|_{U} \otimes \wedge(Z)$, while the range is identified with the left $\wedge\left(\mathrm{W}_{H}^{*}\right)$-invariants in the range of $\Omega_{U}^{\prime}$. One will see that the latter is further identified with $\left.\pi_{*} \mathcal{F}\right|_{U} \otimes \wedge(\mathrm{Z})$, again. Note $\mathrm{W}_{H}^{*}=\operatorname{Lie}(H)_{1}$, and that $\wedge\left(\mathrm{W}_{H}^{*}\right)$ is now a Hopf super-subalgebra of hy $(H)$; it is supposed to act on the last mentioned range through the right multiplication on hy $(H)$. Since hy $(H) \otimes_{\wedge\left(\mathrm{W}_{H}^{*}\right)} \mathbb{k}=\operatorname{hy}\left(H_{\text {red }}\right)$, it follows that the left $\wedge\left(\mathrm{W}_{H}^{*}\right)$-invariants thus coincide with

$$
\operatorname{Hom}_{\mathrm{hy}\left(H_{\mathrm{red}}\right)}\left(\mathrm{hy}\left(H_{\mathrm{red}}\right),\left.\pi_{*} \mathcal{F}\right|_{U} \otimes \wedge(\mathrm{Z})\right)=\left.\pi_{*} \mathcal{F}\right|_{U} \otimes \wedge(\mathrm{Z}) .
$$

Now, the restriction of $\Omega_{U}^{\prime}$ in question is seen to be the identity map on $\left.\pi_{*} \mathcal{F}\right|_{U} \otimes \wedge(Z)$, whence it is isomorphic, as desired.

$(2)$ Regard $\mathbb{k}$ as the trivial module over hy $\left(H_{\text {red }}\right)$ and over $K$. Since the hy $(H)$-invariants in the range of $\Omega_{U}$ equal $\operatorname{Hom}_{\mathrm{hy}\left(H_{\mathrm{red}}\right)}\left(\mathbb{k},\left.\wedge(\mathrm{Z}) \otimes \pi_{*} \mathcal{F}\right|_{U}\right)$, one sees from (7.2) that $\left(\Omega_{U}\right)^{J}$ coincides with

$\mathcal{O}\left(\pi^{-1}()\right)^{J} \stackrel{\simeq}{\longrightarrow} \operatorname{Hom}_{K}\left(\mathbb{k},\left.\wedge(\mathbf{Z}) \otimes \pi_{*} \mathcal{F}\right|_{U}\right)=\left(\left.\wedge(\mathbf{Z}) \otimes \pi_{*} \mathcal{F}\right|_{U}\right)^{K}=\left.\wedge(\mathbf{Z}) \otimes \mathcal{F}_{Q}\right|_{U}$. 
Proof of Theorem 7.3. Since the first half of Part 1 is proved by Proposition 7.8 (2), it suffices to prove Part 2, from which the rest follows easily.

Suppose that the situation is as above the proof. Define a morphism of manifolds by

$$
\sec _{U}: U \rightarrow \pi^{-1}(U), \sec _{U}(u)=\operatorname{triv}_{U}(u, e) .
$$

By Proposition 7.8 (2) we can identify $\left.\mathcal{O}_{G / H}\right|_{U}$ with $\left.\wedge(Z) \otimes \mathcal{F}_{Q}\right|_{U}$ through $\left(\Omega_{U}\right)^{J}$. A close look at the construction of the isomorphism $\Omega_{U}$ above, without taking care of the relevant $H_{\text {red-actions, gives an isomorphism }}$

$$
\left.\mathcal{O}\right|_{\pi^{-1}(U)} \stackrel{\simeq}{\longrightarrow} \operatorname{Hom}_{\mathrm{hy}\left(H_{\mathrm{red}}\right)}\left(\mathrm{hy}(H),\left.\wedge(\mathrm{Z}) \otimes \mathcal{F}\right|_{\pi^{-1}(U)}\right)
$$

of sheaves on $\pi^{-1}(U)$. The last $\left.\mathcal{F}\right|_{\pi^{-1}(U)}$ on the right-hand side may be replaced with the direct image of $\mathcal{F}_{U \times H_{\text {red }}}$ under triv ${ }_{U}$. Let us consider the sheaf-isomorphism obtained after the replacement. Choose an arbitrary element $(x, h) \in U \times H_{\text {red }}$, and let $f=\sec _{U}(x), g=\operatorname{triv}_{U}(x, h)$, and so $g=$ $f h$. Then one sees that the sheaf-isomorphism gives rise to the isomorphism between the completed stalks

$$
\hat{\mathcal{O}}_{g} \stackrel{\simeq}{\longrightarrow} \wedge(\mathrm{Z}) \otimes \hat{\mathcal{F}}_{Q, x} \hat{\otimes} \operatorname{Hom}_{\text {hy }\left(H_{\text {red }}\right)}\left(\operatorname{hy}(H), \hat{\mathcal{F}}_{H_{\text {red }}, h}\right),
$$

which decomposes so as

$$
\begin{aligned}
\hat{\mathcal{O}}_{g} \stackrel{\hat{\Delta}_{f, h}}{\longrightarrow} \hat{\mathcal{O}}_{f} \hat{\otimes} \hat{\mathcal{O}}_{h} \stackrel{\hat{\Sigma}_{U, f} \hat{\otimes} \text { cano }}{\longrightarrow} & \wedge(\mathrm{Z}) \otimes \hat{\mathcal{F}}_{f} \hat{\otimes} \hat{\mathcal{O}}_{H, h} \\
& \stackrel{\mathrm{id}_{\wedge(\mathrm{z})} \otimes\left(\widehat{\sec _{U}^{*}}\right)_{x} \hat{\otimes} \hat{\eta}_{H, h}}{\longrightarrow} \wedge(\mathrm{Z}) \otimes \hat{\mathcal{F}}_{Q, x} \hat{\otimes} \operatorname{Hom}_{\mathrm{hy}\left(H_{\mathrm{red}}\right)}\left(\mathrm{hy}(H), \hat{\mathcal{F}}_{H_{\mathrm{red}}, h}\right),
\end{aligned}
$$

where cano $: \hat{\mathcal{O}}_{h} \rightarrow \hat{\mathcal{O}}_{H, h}$ is the canonical map arising from the inclusion $G \supset H$, and $\eta_{H}: \mathcal{O}_{H} \stackrel{\simeq}{\longrightarrow} \operatorname{Hom}_{\mathrm{hy}\left(H_{\mathrm{red}}\right)}\left(\mathrm{hy}(H), \mathcal{F}_{H_{\mathrm{red}}}\right)$ is the canonical sheafisomorphism for $H$, as given by Proposition 6.11 (1); the last two sheaves are identified through $\eta_{H}$. It follows that $\pi^{-1}(U)$ is right $H$-stable in $G$, and the sheaf-isomorphism in question is associated with an isomorphism

$$
\operatorname{Triv}_{U}:\left(U,\left.\mathcal{O}_{G / H}\right|_{U}\right) \times H \stackrel{\simeq}{\longrightarrow}\left(\pi^{-1}(U),\left.\mathcal{O}\right|_{\pi^{-1}(U)}\right)
$$

of right $H$-equivariant super-manifolds, which is seen to have the two properties described in the theorem.

Remark 7.9 (added in revision). The referee kindly informed the authors of the article [4 by R. Fioresi et al., which proves an analogous result (see [4. Section 3]) of our Theorem 7.3 in the (real) $C^{\infty}$-situation. Their proof, which uses the Frobenius Theorem [1, Chapter 4], constructs the quotient $G / H$ and proves that $G \rightarrow G / H$ is a principal super $H$-bundle (in the $C^{\infty}$-situation). But seemingly, it does not prove the property that any non-super trivialization is liftable to a super trivialization; see Remark 7.4 (1). We remark that our proof slightly modified proves their result including that liftability; the modification uses the fact that (real) Lie groups uniquely turn into real analytic groups, and their finite-dimensional representations are necessarily analytic.

\section{ACKNOWLEDGMENTS}

The authors gratefully acknowledge that the work was supported by JSPS Grant-in-Aid for Scientific Research (C), 26400035 and 17K05189. 


\section{REFERENCES}

[1] C. Carmeli, L. Caston, R. Fioresi, Mathematical Foundations of Supersymmetry, EMS Series of Lectures in Mathematics, European Math. Soc., Zürich, 2011.

[2] C. Carmeli, R. Fioresi, Superdistributions, analytic and algebraic super HarishChandra pairs, Pacific. J. Math. 263 (2013), 29-51.

[3] P. Deligne, J.-W. Morgan, Notes on supersymmetry (following Joseph Bernstein), in: Quantum fields and strings: a course for mathematicians, vols. 1, 2 (Princeton, NJ, 1996/1997), Amer. Math. Soc., Providence, 1999, pp.41-97.

[4] R. Fioresi, M. A. Lledó, V. S. Varadarajan, The Minkowski and conformal superspaces, J. Math. Phys. 48, 113505 (2007).

[5] F. Gavarini, Global splittings and super Harish-Chandra pairs for affine supergroups, Trans. Amer. Math. Soc. 368 (2016), 3973-4026.

[6] G. Hochschild, G. D. Mostow, Complex analytic groups and Hopf algebras, Amer. J. Math. 91 (1969), 1141-1151.

[7] B. Kostant, Graded manifolds, graded Lie theory, and prequantization, Lecture Notes in Mathematics vol. 570, Springer-Verlag, Berlin/Heidelberg/New York, 1977, pp.177-306.

[8] J.-L. Koszul, Graded manifolds and graded Lie algebras, Proceedings of the International Meeting on Geometry and Physics (Florence, 1982), pp.71-84, Pitagora, Bologna, 1982.

[9] A. Masuoka, Formal groups and unipotent affine groups in non-categorical symmetry, J. Algebra 317 (2007), 226-249.

[10] A. Masuoka, The fundamental correspondences in super affine groups and super formal groups, J. Pure Appl. Algebra 202 (2005), 284-312.

[11] A. Masuoka, Harish-Chandra pairs for algebraic affine supergroup schemes over an arbitrary field, Transform. Groups 17 (2012), no. 4, 1085-1121.

[12] A. Masuoka, Hopf algebraic techniques applied to super algebraic groups, Proceedings of Algebra Symposium (Hiroshima, 2013), pp. 48-66, Math. Soc. Japan, 2013; available at arXiv: $1311.1261 \mathrm{v} 2$.

[13] A. Masuoka, T. Shibata, Algebraic supergroups and Harish-Chandra pairs over a commutative ring, Trans. Amer. Math. Soc. 369 (2017), 3443-3481.

[14] A. Masuoka, T. Shibata, On functor points of affine supergroups, J. Algebra 503 (2018), 534-572.

[15] A. Masuoka, Y. Takahashi, Geometric construction of quotients G/H in supersymmentry, Transform. Groups, in press; preprint arXiv:1808.05753v4.

[16] A. Masuoka, A. N. Zubkov, Solvability and nilpotency for algebraic supergroups, J. Pure Appl. Algebra 221 (2017), 339-365.

[17] S. Montgomery, Hopf algebras and their actions on rings, CBMS Conf. Series in Math., vol. 82, Amer. Math. Soc., Providence, RI, 1993.

[18] J. P. Serre, Lie algebras and Lie groups, 2nd. ed., Lec. Notes in Math., vol. 1500, Springer-Verlag, Berlin/Heidelberg, 1992.

[19] T. Schmitt, Regular sequences in $\mathbb{Z}_{2}$-graded commutative algebra, J. Algebra $\mathbf{1 2 4}$ (1989), 60-118.

[20] I. R. Shafarevich, Basic Algebraic Geometry, Die Grundlehren der mathematischen Wissenschaften, Springer, Berlin/Heidelberg/New York, 1972.

[21] M. E. Sweedler, Hopf Algebras, W. A. Benjamin, Inc., New York, 1969.

[22] M. Takeuchi, Tangent coalgebras and hyperalgeras I, Japanese J. of Math. 42 (1974), $1-143$.

[23] M. Takeuchi, Topological coalgebras, J. Algebra 97 (1985), 505-539.

[24] V. S. Varadarajan, Introduction, in: S. Ferarra, R. Fioresi, V. S. Varadarajan (eds.), Supersymmetry in Mathematics and Physics, Lec. Notes in Math., vol. 2027, SpringerVerlag, Heidelberg/Dordrecht/London/New York, 2011, pp.1-15.

[25] E. G. Vishnyakova, On complex Lie supergroups and split homogeneous supermanifolds, Transform. Groups 16 (2011), no. 1, 265-285.

[26] W. C. Waterhouse, Introduction to Affine Group Schemes, Graduate Texts in Mathematics 66, Springer, New York/Heidelberg/Berlin, 1979. 
[27] T. Wedhorn, Manifolds, Sheaves and Cohomology, Springer Studium Mathematik, Springer Spektrum, 2016.

Mitsukazu Hoshi, Institute of Mathematics, Graduate School of Pure and Applied Sciences, University of Tsukuba, Ibaraki 305-8571, Japan

E-mail address: m-hoshi@math.tsukuba.ac.jp

Akira Masuoka, Institute of Mathematics, University of Tsukuba, Ibaraki 305-8571, JAPAN

E-mail address: akira@math.tsukuba.ac.jp

Yuta Takahashi, Graduate School of Pure and Applied Sciences, University of Tsukuba, Ibaraki 305-8571, Japan

E-mail address: y-takahashi@math.tsukuba.ac.jp 